\title{
Femmes, villes et environnement
}

\section{Textes réunis par \\ Yvonne Preiswerk \\ Isabelle Milbert}
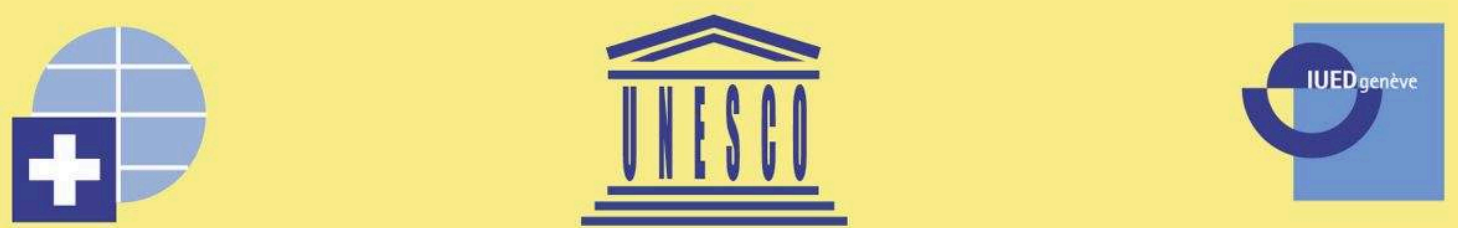

Commission nationale suisse pour l'Unesco, Berne

DDC, Direction de la coopération au développement, de l'aide humanitaire et de la coopération technique avec l'Europe centrale et orientale Département fédéral des affaires étrangères, Berne

IUED, Institut universitaire d'études du développement, Genève

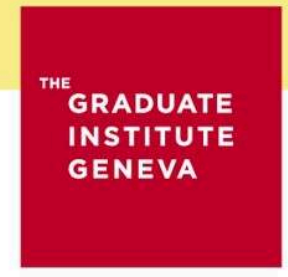

$-$

INSTITUT DE HAUTES ÉTUDES INTERNATIONALES ET DU DÉVELOPPEMENT

GRADUATE INSTITUTE

OF INTERNATIONAL AND DEVELOPMENT STUDIES

\section{GENRE ET DÉVELOPPEMENT | RENCONTRES}




\section{Graduate Institute Publications}

GRADUATE

INSTITUTE

GENEVA

\section{Femmes, villes et environnement}

Isabelle Milbert et Yvonne Preiswerk (dir.)

DOI : 10.4000/books.iheid.5566

Éditeur : Graduate Institute Publications

Lieu d'édition : Genève

Année d'édition : 1995

Date de mise en ligne : 5 juillet 2016

Collection: Genre et développement. Rencontres

EAN électronique : 9782940503728

\section{OpenEdition}

Books

https://books.openedition.org

Édition imprimée

Nombre de pages : 229

Référence électronique

MILBERT, Isabelle (dir.) ; PREISWERK, Yvonne (dir.). Femmes, villes et environnement. Nouvelle édition [en ligne]. Genève : Graduate Institute Publications, 1995 (généré le 28 janvier 2022). Disponible sur Internet : <http://books.openedition.org/iheid/5566>. ISBN : 9782940503728. DOI : https://doi.org/ 10.4000/books.iheid.5566.

(c) Graduate Institute Publications, 1995

Creative Commons - Attribution - Pas d'Utilisation Commerciale - Pas de Modification 3.0 non transposé - CC BY-NC-ND 3.0 


\section{RÉSUMÉS}

Cet ouvrage veut faire écho aux paroles des femmes qui, dans l'expérience de la vie urbaine, doivent s'unir pour inventer, innover, participer comme partenaires avec les institutions - donc avec les hommes - à un environnement souvent hostile, dégradé et malsain. Il met en perspective le couple ville/environnement lié à la question des femmes. Lorsqu'il s'agit de développement urbain et d'environnement, tant au niveau micro (les ménages) que macro (la société), les rôles des hommes et des femmes devraient être interdépendants. Pourtant, les structures reproduisent de fait les inégalités...

\section{ISABELLE MILBERT (DIR.)}

Juriste et urbaniste, IUED, Genève.

\section{YVONNE PREISWERK (DIR.)}

Anthropologue, IUED, Genève; Université de Fribourg. 


\section{Femmes, villes et environnement}

Textes réunis par Yvonne Preiswerk et Isabelle Milbert

229 pages, 1995

(coédition avec la DDC et la Commission nationale suisse pour l'UNESCO)

\section{Table des matières}

Avant-propos

Jean Luc Maurer

Introduction

Yvonne Preiswerk

\section{Apports théoriques}

Les structures urbaines existantes sont-elles une entrave à l'égalité entre les sexes?

Ursula Paravicini

Quelles villes pour les femmes dans les pays du Sud au XXIe siècle?

Françoise Lieberherr

Du bon genre... ou des relations de genre appropriées

Christine Verschuur

Processus de décentralisation municipale et environnement urbain : quelles perspectives pour les femmes

Isabelle Milbert

Migrer c'est résister : à propos des femmes en migration

Laurent Monnier

La ville au féminin et au masculin

Rodrigo Vidal Rojas

Une nouvelle approche du partage de l'emploi: le rôle des femmes

Andràs November

Femmes et hommes sur le terrain: Approches empiriques à travers les continents

$$
>\text { Afrique }
$$

Les femmes algériennes: spectatrices ou actrices dans la ville?

Fadela Chaib 
Women in conflict situation: Gender issues in transition from war to democracy in Namibia and Mozambique

Marni Pigott

Répartition des rôles entre hommes et femmes en zone péri-urbaine au Mozambique Margarita Mejia

Division sexuelle du travail et gestion de l'environnement: la valorisation de la femme Kadidia Tall

De la nécessité de réinventer de nouveaux rapports hommes-femmes en Afrique aujourd'hui Fatima Meite et Aminata Traore

Les activités féminines dans l'environnement urbain au Sénégal

Emmanuel Ndione

Réseaux de femmes et accès à la décision: l'exemple des groupements féminins au Sénégal Cheikh Badiane

L'éducation socio-familiale : la place du couple

Pape Diouf

\section{$>$ Amérique latine et centrale}

Menuisières, charpentières, maçons... La coopérative de femmes de Mulukuku au Nicaragua Grethel Sequeira Montoya

\section{Les femmes et un mouvement populaire d'habitations : Sao Paulo, Brésil}

Jeanne Bisilliat

La maison des autres, «A casa dos outros » : L'emploi domestique à Recife, Nordeste du Brésil

Marie Anderfuhren

\section{$>$ Inde}

The Thin Edge of the Wedge-pushing Accountability into Public System. Experience from Bombay-India

Pratima Panwalkar

Principle Centred Development - A New Paradigm Habitat

Sheba Hussain

Women Entrepreneurship Improving The Quality of Life

Runa Banerjee 


\section{AVANT-PROPOS}

La diversité de nos activités, que ce soit l'enseignement, la recherche ou encore la gestion de projets opérationnels de développement, donne à notre institution un champ extrêmement large, riche et varié dans lequel s'inscrivent les réflexions sur les rapports sociaux entre hommes et femmes et l'analyse de genre aujourd'hui.

Depuis quelques années, notre volonté a été de souligner la problématique « genre »dans toutes les études sur le développement dans une perspective transversale touchant les différentes disciplines abordées dans notre maison : l'économie, l'anthropologie, la sociologie politique. Tous ces champs, appliqués aux études rurales ou urbaines, aux questions de l'environnement, aux problèmes de santé et à toutes les études sectorielles, s'articulent et s'entrecroisent avec l'enseignement, la recherche et les projets de développement. Ainsi, certains séminaires reliés à l'économie et à l'environnement ont été enrichis par l'éclairage des rapports hommes/femmes.

La problématique de l'urbain et du développement des villes ne pouvait faire l'impasse sur l'approche genre ; il en va de même pour tous les enseignements et toute la recherche inter et transdisciplinaire se rapportant à l'éducation, aux zones rurales ou marginales, à l'industrialisation de l'Asie du Sud-Est, par exemple.

S'il est inutile d'énumérer ici tous les enseignements, recherches, activités opérationnelles, colloques, groupes de travail et travaux d'étudiants avancés entrepris dans les champs croisés des disciplines de l'iuéd, il est en revanche primordial de souligner notre souci et notre volonté d'un éclairage transversal permanent que permettent la problématique genre et celle des rapports sociaux équilibrés entre les sexes. Le développement est aujourd'hui l'affaire de tous et concerne les femmes comme les hommes en tant que partenaires d'un même projet de société.

Que soient ici remerciés ceux qui veillent à ce juste équilibre mais aussi ceux qui, par leurs apports scientifiques, culturels et financiers, dont la DDA et l' UNESCO, permettent que des colloques et séminaires viennent enrichir et élargir notre réflexion vers un public plus grand. C'est l'objet de la présente publication «Femmes, villes et environnement ». 


\section{INTRODUCTION}

Les récentes conférences internationales avouent l'importance du mal-être et de l'injustice que rencontrent les femmes dans le monde: pour cette moitié de l'humanité, les inégalités sont tenaces. Il est urgent que non seulement les femmes soient entendues mais qu'elles aient accès aux droits des personnes les plus élémentaires accordés à tout être humain. Il serait toutefois inconvenant de ne parler que d'elles alors que la guerre, les crises, le mal-développement, la pauvreté, la maladie, frappent un peu partout les enfants, les hommes et les femmes de la planète davantage au Sud qu'au Nord, à l'Est qu'à l'Ouest. Jamais peut-être la puissance des uns n'a fait apparaître autant l'impuissance des autres. Pourtant, dans chaque village ou ville, dans chaque pays, les femmes et les hommes s'unissent pour affronter, avec courage et détermination, leurs difficultés d'exister. Ils devraient le faire ensemble, avec les mêmes droits et les mêmes devoirs. Or, dans ce partage et plus particulièrement dans celui de l'accès aux décisions, les femmes subissent l'inégalité ancestrale due à leur sexe; elles sont le plus souvent en marge des événements qui déterminent leur vie quotidienne, même si elles font montre en permanence d'une extraordinaire vitalité pour satisfaire leurs besoins fondamentaux, ceux de leurs familles, de leurs sociétés.

Cet ouvrage veut faire écho aux paroles des femmes qui, dans l'expérience de la vie urbaine, doivent s'unir pour inventer, innover, se faire entendre, participer comme partenaires avec les institutions, donc avec les hommes, à un environnement souvent hostile, dégradé, malsain et inacceptable. La revendication d'une qualité de vie meilleure pour tous légitime une mobilisation sur tous les fronts. Mais, plus exposées dans les guerres, dans les crises, dans les situations difficiles, les femmes souffrent aussi de handicaps sociaux évidents: manque de droits, peu d'accès à l'éducation, violences physiques et morales. Elles restent souvent en marge des richesses, des ressources, de l'accès au sol, ce qui paralyse leur destin. En outre, les peuplements suburbains, la dislocation familiale, l'habitat de survie, les plans d'urbanisation ne permettent guère un partage des tâches entre hommes et femmes et entre les générations.

Aborder le problème des villes et de l'environnement est somme toute assez classique. Analyser les rapports sociaux entre les sexes, les inégalités entre hommes et femmes est à l'ordre du jour dans les institutions et les études universitaires de tous bords. En revanche, mettre en perspective, comme hypothèse de travail, le couple ville/environnement lié à la question des femmes surprend doublement par son nouvel éclairage.

D'une part, les inégalités de "toujours" entre hommes et femmes s'éclairent de façon originale dans le contexte urbain. On y perçoit comment l'un et l'autre sexe vit de manière différente, travaille différemment, agit ou subit différemment les problèmes urbains de l'environnement. Dans cette perspective, la ville est prise comme cadre de vie avec ses répercussions propres.

D'autre part, en prenant la ville non plus comme contexte mais comme "productrice" de rapports sociaux et de sens, on perçoit comment l'urbain distille et reproduit de façon spécifique les inégalités entre hommes et femmes. 
Il serait fort heureux à l'avenir de pouvoir lier en une seule problématique la "ville contexte" et la "ville acteur". Cette synthèse est prématurée au stade actuel des recherches. Mais les matériaux révélés ici permettent d'augurer de passionnantes découvertes. Pour l'instant en tous cas, l'inventaire des questions se montre foisonnant.

Lorsqu'il s'agit de développement urbain et d'environnement, tant au niveau micro (les ménages) que macro (la société), les rôles des hommes et des femmes devraient être interdépendants mais les structures reproduisent de fait les inégalités. En est-il de même au Nord qu'au Sud? Peut-on réfléchir à partir de la même hypothèse?

En essayant d'appréhender de façon globale le thème croisé de "Femmes, villes, environnement", nous avons posé à nos inter-locuteurs l'interrogation majeure suivante:

- partant du principe que la ville est le lieu de transformations accélérées,

- partant du constat que les franges les plus pauvres des populations urbaines vivent dans l'urgence, c'est-à-dire sans égard particulier pour la conservation à long terme du milieu ou de l'environnement (comme le faisaient les communautés rurales soucieuses de "visée écologique pour les générations futures"),

- partant de la constatation presque générale que les femmes cumulent les fonctions domestiques et les fonctions de production des conditions de survie,

- partant surtout du fait que l'urbain et l'environnement reproduisent des inégalités de sexe,

est-il dès lors possible de porter un regard nouveau sur le lien femme/homme et environnement en zone urbaine?

Cette perspective nouvelle impose des questions plus pertinentes, plus approfondies et plus ponctuelles sur ce lien revisité. Les voici telles que nous les avons posées à nos partenaires de cette réflexion:

- Comment les urbains pensent-ils leur environnement, la qualité de leur vie, quels sont leurs possibilités d'actions? Y a-t-il une visée commune entre les hommes et les femmes, et comment s'articulent ces différences?

- De quelle manière l'environnement urbain agit-il sur le comportement de la femme - et des hommes ainsi que de la relation entre eux - individuellement et collectivement? Assiste-t-on à de nouveaux types de violences (réelles ou symboliques)? A cet égard y a-t-il des mentalités et des comportements nouveaux selon les générations?

- Dans les pays en guerre comment se répartissent les multiples rôles de survie entre hommes et femmes et qu'en est-il dans les périodes d'accalmie ou de retour à la paix?

- Quelle est l'image de la nature - quels mythes, quelles réalités - lorsqu'on habite en zone urbaine? Existe-t-il des réminiscences par rapport à la nature au village, par exemple ressentir la nécessité de voir des arbres, des fleurs, d'avoir des animaux "non utilitaires"? 
- Quelles sont les possibilités d'accès aux structures de décision? Quelles sont les modalités de négociation avec le pouvoir que peuvent instaurer les femmes quant à l'environnement construit (quartiers, maisons, lieux de service, transports)?

- Quel est l'impact des femmes sur les prises de décisions pour gérer l'environnement au niveau des pratiques quotidiennes (recyclage, déchets, latrines, etc.)?

- Quels moyens ont-elles de prendre en charge des alternatives de productions de biens qui seraient respectueuses de l'environnement (économies parallèles, réseaux informels)? Y a-t-il lieu de se poser cette question pour le Tiers Monde? Quels sont les réseaux de solidarité qui s'instaurent ou peuvent s'instaurer pour améliorer le quotidien dans ce cadre?

- Quelle est leur marge de créativité et quel soutien ont-elles de la part des hommes pour des innovations qui améliorent leurs qualités de vie? Qui procure l'argent nécessaire (les hommes ou les femmes elles-mêmes)?

- Comment les femmes conscientisent-elles leurs enfants à une gestion acceptable du milieu (qualité de vie, de l'eau, accès au marché, jardinage)?

- Y a-t-il une division des rôles sociaux de sexe dans les questions d'environnement ou est-ce une "question occidentale"?

- Les agences de coopération peuvent-elles mettre en oeuvre des projets qui donnent la priorité aux femmes et à l'environnement? Et dans ce cas comment le partage des droits et devoirs entre hommes et femmes concernés peut-il être géré?

Cette liste longue et incomplète de questions très pratiques ouvre sur deux constats fondamentaux. Le premier est que la réalité transmise par les différentes expériences ponctuelles décrites dans cet ouvrage est infiniment plus riche et éclairante qu'on ne pouvait l'imaginer. La créativité humaine, poussée parfois dans ses retranchements, déborde de vitalité, d'inventivité et de détermination. Tous ces récits décrivant l'engagement et les liens de solidarité appellent l'admiration et notre soutien inconditionnel.

Le deuxième constat prévaut plutôt pour les sociétés développées. Les réflexions théoriques qui jalonnent nos connaissances en matière d'histoire, d'urbanisme, de migrations, d'échanges économiques entre autres, ne tiennent pas assez compte des rapports sociaux entre hommes et femmes qui pourtant permettent de manière tout à fait novatrice de revisiter ces disciplines pour penser demain.

Nous souhaitons par cet ouvrage enrichir le vaste débat d'idées, au Nord comme au Sud, sur les villes et l'environnement, et sur le rôle que les femmes peuvent y jouer. 


\section{LES APPORTS THEORIQUES}

Les réflexions théoriques sur les rapports sociaux entre les sexes, les études sociologiques et anthro pologiques liées aux pratiques de la vie quotidienne, les interrogations sur l'espace urbain, les grandes villes et leurs périphéries, mais aussi les alternatives possibles quant au partage du travail sont nées dans les sociétés développées.

C'est une réalité à laquelle il s'agit de faire face sans aucune sorte de prétention ou de fatuité. Elle oblige à la précaution et, en conséquence, au souci de n'avoir aucun jugement de valeur parti culier et encore moins d'ordre biérarchique face aux pratiques d'autres sociétés et face aux approches empiriques si riches qui nourrissent notre réflexion commune. Ces apports théoriques ne s'adaptent pas forcément à d'autres peuples, d'autres mentalités, d'autres visions du monde, mais ils sont une invitation pressante à établir une problématique qui soit propre à chaque société, dans un effort de comparaison et de confrontation.

Afin de ne pas proposer de synthèse hâtive, le questionnement théorique présenté dans ces textes reflète une hétérogénéité et une diversité où se côtoient contradictions, querelles d'école, points de vue masculins et féminins qui ouvrent sur une pensée éclatée propre à alimenter les débats futurs. Il nous a semblé important que s'expriment ici en toute liberté des tendances que certains auteurs pensent inconciliables. 








\title{
LES STRUCTURES URBAINES EXISTANTES SONT-ELLES UNE ENTRAVE A L'EGALITE DES SEXES?
}

\author{
URSULA PARAVICINI
}

Contrairement à la plupart des articles réunis dans cette publication, notre contribution se situe dans le contexte des pays de l'Europe de l'Ouest et s'inscrit dans le cadre du débat féministe qui connaît ces dernières années, surtout en Allemagne, un regain d'intérêt. De nombreux articles et livres publiés par des femmes architectes et urbanistes dénoncent notamment les problèmes d'insécurité pour femmes et enfants dans l'espace public ainsi que l'inadéquation des logements par rapport aux modes de vie en pleine transformation.

Egalité utopique? C'est une des interrogations centrales posées dans cet ouvrage. Nous nous intéressons ici plus particulièrement à la question suivante: les structures sociales et spatiales des villes en Europe constituent-elles une entrave à l'égalité entre les sexes, au point de la rendre utopique? De manière plus générale, sont-elles un frein à la modernisation de notre société et à l'actualisation des modes de vie?

Ces questions doivent être mises en relation avec la rapidité et l'ampleur avec lesquelles s'opèrent actuellement les transformations dans tous les domaines de la vie sociale. Les procès de modernisation ne sont pas uniquement portés par le progrès technique et industriel, mais tout autant par des poussées de transformations dans le domaine de la reproduction et dans la sphère privée qui remettent profondément en question le cadre temporel et spatial hérité de la société fordiste.

Parmi ces transformations, l'insertion massive des femmes dans le salariat représente une lame de fond du changement social. Elle traduit l'aspiration d'un nombre croissant de femmes à vouloir sortir de l'étroitesse de la sphère domestique et participer pleinement à la vie sociale. Cette aspiration des femmes s'inscrit dans un procès d'émancipation et constitue le moteur d'une dynamique sociale irréversible.

\section{LA MONTÉE DE NOUVELLES ASPIRATIONS FÉMININES}

Nous nous fondons sur le fait que les processus de transformation profonde observés dans la famille depuis les années 1960 sont la conséquence de la croissance rapide du nombre de femmes exerçant une activité professionnelle. Les statistiques nationales européennes indiquent des taux différents d'activité féminine avec des hauts dans les pays scandinaves et des bas dans les pays méditerranéens. C'est toutefois le même procès de transformation qui est en cours dans tous les 
pays européens: toujours plus de femmes exercent une activité professionnelle; la part de celles qui interrompent leur activité diminue constamment, même si elles se marient et que leurs enfants sont jeunes ou en âge scolaire (OCDE, 1985).

En France par exemple, où le taux d'activité féminine est à peine supérieur à la moyenne européenne, le nombre des femmes exerçant une activité professionnelle, de moins de 7 millions qu'il était en 1960, a augmenté à plus de 10 millions aujourd'hui, passant de moins de 34\% à environ $43 \%$ de la population active dans son ensemble. De manière schématique, on peut considérer que, pendant les années 1960, deux tiers des femmes restaient à la maison et seulement un tiers exerçait une activité professionnelle; la proportion s'est aujourd'hui inversée: un tiers d'entre elles restent à la maison, deux tiers exercent une activité professionnelle.

Au niveau scientifique, on reconnaît désormais la valeur heuristique particulière des travaux de recherche qui traitent de l'activité professionnelle des femmes. De nombreux auteurs (Perrot, 1984; Comaille, 1993) ont montré que les gender studies (à savoir la recherche portant sur les relations entre les sexes) donnent lieu à l'analyse des processus d'adaptation réciproques entre les systèmes de production, les structures familiales et l'organisation de la sphère privée. Les catégories habituelles de travail et de "non-travail" (ou travaux ménagers) ainsi que la division sociale du travail doivent dans cette perspective être repensées en tenant compte des différences spécifiques à chacun des sexes. Une telle approche permet d'affirmer que l'intégration massive des femmes dans le monde du travail et les procès de transformation qui en résultent dans la vie quotidienne, contribuent de manière décisive à la modernisation de la société.

Les raisons qui expliquent l'augmentation du travail professionnel des femmes sont de plusieurs ordres; de toute évidence elles sont, entre autres, d'ordre économique. Parmi les procès de transformation portés par le progrès industriel et technique, la pénétration croissante des produits industriels dans les ménages en est l'une des raisons structurelles (Schwartz-Cowan, 1983).

Dans une première phase, au temps de Ford et du développement de la production de masse, les appareils électroménagers firent irruption dans la sphère domestique. Une partie des travaux ménagers, comme par exemple la lessive, connut une rationalisation importante. Plus tard, à partir des années 1970, mais surtout depuis les années 1980 et encore aujourd'hui, une partie croissante des travaux domestiques ont été externalisés. Les produits industriels les plus divers, écoulés sur le marché à un coût extrêmement compétitif puisque fabriqués dans un contexte de haute productivité, ont rendu de nombreuses tâches ménagères exécutées dans la sphère privée de moins en moins rentables.

A titre d'exemple, la production ménagère d'habits tend à être remplacée par la confection, la préparation des aliments cède en partie la place aux produits précuits ou déjà préparés, et des articles jetables (langes, mouchoirs en papier...) se substituent aux services qui auparavant requéraient un travail conséquent. La signification économique du travail ménager se trouve désormais renversée et la répartition du travail entre les sexes remise en question. Alors que durant les années 1960, le niveau de vie d'une famille était plus élevé si le revenu de l'homme était suffisant pour permettre que la femme se consacre entièrement au ménage, il est actuellement, pour une famille, plus avantageux sur le plan matériel si la femme exerce également une activité professionnelle.

Le développement rapide des offres d'emploi dans le secteur des services est une autre raison de 
l'augmentation du nombre de femmes qui exercent une activité professionnelle. Les capacités dont les salariés de ce secteur doivent faire preuve correspondent à celles qui sont nécessaires dans la sphère domestique et pour lesquelles une femme est déjà préparée par son éducation spécifique. Comme pour le travail domestique, il est déterminant d'être en mesure d'exercer côte à côte et simultanément plusieurs activités et rythmes de travail différents. De manière analogue à l'attention que requièrent les enfants et les jeunes, il faut avant tout savoir écouter les autres clients ou collaborateurs - de manière à assurer la qualité des services. Ce sont ces compétences sociales, dont les femmes disposent pour des raisons historiques, qui contribuent à la proportion élevée des travailleuses féminines dans le secteur des services en expansion constante.

Mais les raisons de la participation croissante des femmes au monde du travail sont également d'ordre social et culturel. On peut affirmer que les nouvelles aspirations féminines s'inscrivent dans un procès de civilisation dans la longue durée (Elias, 1969). L'accès à la formation et à la culture, aujourd'hui allant de soi dans l'ensemble des pays européens pour un nombre toujours croissant de jeunes femmes, est décisif pour une nouvelle prise de conscience de la part de beaucoup d'entre elles. Plus le niveau de formation est élevé, plus l'attente de pouvoir s'extraire de l'étroitesse de la sphère privée pour travailler et jouer un rôle à tous les niveaux de la vie sociale est grand.

La révolution dans le contrôle des naissances - dont on sous-estime aujourd'hui encore la signification - a également participé au processus de modernisation de notre société de manière émancipatoire. En pouvant librement choisir de devenir mères, le nombre de leurs enfants et le moment des naissances, les femmes sont en mesure de planifier de manière plus consciente leur vie et entre autres leur activité professionnelle (Beck-Gernsheim, 1993). Cette révolution n'est pas attribuable uniquement au progrès médico-scientifique, elle renvoie également à la volonté collective d'un grand nombre de femmes en faveur de la reconnaissance du contrôle des naissances sur le plan politique. Ces raisons d'ordre social et culturel ne sont pas spécifiques aux pays d'Europe mais les conditions y sont particulièrement favorables pour les femmes.

\section{LES TRANSFORMATIONS DANS LA SPHÈRE PRIVÉE COMME VECTEUR} DE MODERNISATION

L'intégration croissante des femmes dans le monde du travail s'accompagne de transformations profondes dans la sphère familiale et dans le rapport entre les sexes. Les transformations dans les compositions des ménages sont les mieux connues, puisqu'elles sont quantifiables. Alors que la proportion des familles nucléaires constituées de deux ou trois enfants continue de diminuer par rapport aux ménages dans leur ensemble, les ménages composés de couples sans enfants, les ménages monoparentaux et les ménages "en réseau" avec des parents divorcés et des enfants issus de mariages précédents ne cessent d'augmenter. Et il ne s'agit que de la partie visible de l'iceberg.

Tout aussi significatif pour l'avenir, mais moins mis en avant est le procès de transformation de la division du travail entre les sexes au sein de la famille. Nous entendons par là une répartition du travail qui concerne toutes les activités de l'homme et de la femme, tant dans le domaine domestique que dans le domaine professionnel. Contrairement à une opinion trop souvent répandue selon laquelle la division du travail entre les sexes s'est à peine modifiée, nous aimerions insister sur le fait que des formes extrêmement différentes de répartition du travail au sein de la 
famille se sont constituées et existent aujourd'hui simultanément.

Ce qui est nouveau et porteur d'avenir, c'est que dans certaines couches disposant d'un niveau de formation relativement élevé, le travail est souvent, et de plus en plus, partagé. Ceci est d'autant plus fréquent quand il s'agit d'un couple de salariés disposant tous les deux d'horaires de travail flexibles, comme par exemple dans le cas des salariés du secteur public. Parmi ces derniers, la priorité est souvent donnée à une relation égalitaire entre conjoints, ce qui signifie en général des carrières professionnelles sans avancement rapide mais un partage du travail égalitaire (BarrèreMaurisson, 1992).

Il faut par ailleurs souligner que le partage du travail égalitaire au sein de la famille est renforcé par la montée du chômage. Dans un contexte de crise structurelle et de précarisation de l'emploi, la double insertion des conjoints dans le salariat devient une stratégie de plus en plus importante pour éviter la perte complète des revenus.

Par rapport à ces modifications dans la famille et la division du travail entre les sexes, la sphère privée n'apparait pas être tributaire de la tradition, mais un vecteur des procès de modernisation de la société qui annonce un changement de fond dans l'usage social de la ville (Perrinjaquet, Paravicini, 1993).

\section{LES STRUCTURES SOCIALES ET SPATIALES DE NOS VILLES HÉRITÉES DE L'ÈRE FORDISTE}

Face à ces nouvelles aspirations féminines et à l'actualisation des modes de vie, quelle est la signification des structures sociales et spatiales des villes d'Europe de l'Ouest?

L'organisation urbaine existante s'est formée essentiellement pendant la période du développement fordiste de notre société et la rapide croissance des périphéries. Elle est avant tout le résultat d'un procès non planifié marqué cependant par les théories unidimensionnelles du Mouvement moderne qui, à défaut de la maitriser, lui ont conféré l'apparence d'un développement rationnel.

La poussée fonctionnaliste s'est emparée de tous les niveaux de la planification de l'espace: à l'échelle macrospatiale, l'urbanisme fonctionnaliste a mené à terme la séparation géographique des lieux de travail et des lieux d'habitation. A l'échelle microspatiale du quartier et du logement, l'architecture a contribué à créer des univers monofonctionnels dans lesquels chaque activité est déterminée à l'avance par la configuration rigide des espaces.

Bien que les structures urbaines accusent des différences importantes, que ce soit entre le Nord 
et le Sud de l'Europe ou entre grandes et petites agglomérations, les villes sont dans leur ensemble divisées schématiquement en deux parties distinctes: les quartiers centraux d'une part et les zones périphériques d'autre part avec des densités sociales et spatiales fortement contrastées.

Au centre, les quartiers anciens sont caractérisés par leur offre diversifiée en places de travail, en services et magasins, ainsi que par leurs opportunités culturelles et de loisirs. Cette densité d'opportunités économiques et sociales, la spécificité de chaque quartier central, les sédiments historiques et la mémoire collective qui y sont inscrits, donnent lieu le plus souvent à une urbanité de grande qualité.

L'attraction qu'exercent les quartiers centraux sur une population de jeunes professionnels qualifiés, aux revenus élevés, a été documentée en premier aux Etats-Unis. Pour le processus de valorisation spatial et social déclenché par ces couches privilégiées, les chercheurs américains ont forgé la notion de gentrification (Smith, Williams: 1987). De nombreuses études montrent qu'au terme de ce processus, les quartiers centraux deviennent des lieux de résidence des ménages sans enfants (ménages constitués de singles, parmi lesquels de nombreuses femmes-cadres ou de couples à division du travail la plus souvent égalitaire). Simultanément se développe un procès d'exclusion des ménages modestes, insuffisamment solvables pour faire face à la flambée des loyers, dont principalement des personnes âgées, des familles avec enfants et des travailleurs émigrés. L'exclusion de ces couches, leur rejet dans des périphéries éloignées du centre, renforcent la division sociale de la ville et la polarisation de la société.

Contrastant avec les quartiers au centre-ville, les zones périphériques qui s'étendent en auréoles successives vers la campagne environnante se caractérisent par leur mono-fonctionalité. Dans les quartiers résidentiels, qu'il s'agisse des quartiers pavillonnaires de l'entre-deux-guerres, des grands ensembles des années 1960-70 ou de l'habitat périurbain des classes moyennes construit pendant les années 80, le déficit en services et équipements ainsi que l'absence quasi totale d'emplois est la règle.

Dans ces quartiers où la vie sociale s'effiloche, où l'espace public est marqué par une perte de sens évidente et où la présence d'espace vert est sensée compenser tous les désavantages, résident majoritairement des familles avec enfants. Ici la division du travail traditionnelle entre les sexes se trouve généralisée: l'homme est le plus souvent le seul à gagner de l'argent et s'accommode des longs trajets pour aller travailler au centre-ville. La femme se replie sur le foyer pour s'y consacrer à ses enfants et assurer la bonne tenue de la maison; confinée dans un quartier dans lequel la communication sociale est largement absente, la femme au foyer est désignée pour cause comme "veuve verte" (Wilson, 1993).

Pour une minorité de ménages, vivre en banlieue correspond à un choix conscient. C'est le cas des ménages dans lesquels les conjoints décident de privilégier une seule carrière professionnelle - pour ainsi dire toujours celle du mari, souvent cadre moyen ou supérieur dans le secteur productif - et qui optent pour l'accession à la propriété.

Pour une majorité cependant, il s'agit d'un lieu de résidence contraint. Lorsque les revenus familiaux ne permettent pas de payer les loyers élevés au centre-ville, les ménages doivent se contenter d'un domicile en périphérie, alors même que, pour les mères de famille qui désirent concilier charges maternelles et travail professionnel, vivre dans un quartier périphérique pose de graves 
problèmes et ceci pour différentes raisons.

En premier lieu, parce que le choix d'une place de travail se trouve pour elle fortement limité. L'état actuel de la recherche démontre sans équivoque que la mère de famille se voit forcée de privilégier la proximité entre lieux de travail et d'habitat et se résigne à afficher un profil bas dans la recherche d'un emploi de proximité, même s'il ne correspond ni à ses qualifications ni à ses attentes. Avec la difficulté de concilier toutes ses responsabilités domestiques et professionnelles, avec la pression qui pèse sur son budget-temps, elle s'efforce à tout prix de réduire les temps de déplacement.

Dans ces conditions, la structure sociale et spatiale actuelle de la ville, notamment la monofonctionalité des quartiers périphériques et l'éloignement entre lieux de travail et d'habitat, constitue une entrave majeure à l'égalité des chances des femmes dans le monde du travail. Ceci est particulièrement grave pour des femmes seules avec enfants pour qui l'habitat social en périphérie est le plus souvent la seule possibilité d'accéder à un logement décent. La restriction à un marché d'emploi de proximité fort limité entraîne pour ces femmes des grandes difficultés économiques, voire le chômage (Herlyn, 1987). Dans les grands ensembles d'habitat social en périphérie des villes se développent de plus en plus "des poches de pauvreté féminine" (Borst, 1990).

En deuxième lieu, pour celles et ceux qui résident dans un quartier périphérique, une division du travail égalitaire entre conjoints se trouve gravement entravée voire même empêchée. Force est de constater que, même lorsque des conjoints souhaitent un partage égalitaire du travail, la recherche d'emplois à temps partiel pour tous les deux - ce qui leur laisse le temps nécessaire pour une prise en charge partagée du travail domestique - est non compatible avec un temps de déplacement important pour se rendre au travail. Les longs déplacements nécessaires pour atteindre le marché de l'emploi diversifié du centre rend bien plus rationnel un partage selon lequel un des conjoints assume seul les trajets et le travail professionnel, tandis que l'autre prend en charge le travail domestique. La structure urbaine existante fige les modes de vie, s'opposant ainsi à la modernisation de la société.

Nous avons soulevé des questions jusqu'ici à l'échelle de la ville et des quartiers. Quant au logement, la conception fonctionnaliste de l'espace privé s'oppose également à un partage égalitaire du travail domestique. Dans les logements de masse de l'après-guerre réalisés selon les principes hérités du Mouvement moderne, chaque activité est prédéterminée par l'architecte et assignée à un espace précis (Paravicini, 1990). La cuisine-laboratoire par exemple, généralisant le modèle initié à Francfort pendant les années 30, est sensée contribuer - de l'avis des architectes - à la libération de la femme "grâce à la rationalisation du travail ménager" (Taut, 1924). En réalité, les dimensions minimalistes de la cuisine rendent impossible le travail simultané de deux personnes. Autrement dit, la cuisine est définie comme l'espace de travail de la femme et entérine ainsi une répartition inégalitaire du travail entre les sexes.

\section{POUR LE DÉPASSEMENT DU FONCTIONNALISME EN ARCHITECTURE ET URBANISME}

Notre réponse à la première partie de la question posée en introduction est sans ambiguïté: nos structures urbaines existantes constituent une entrave majeure à l'égalité entre les sexes; elles créent des obstacles graves à l'accès des femmes au marché de l'emploi et elles tendent à figer 
une division inégalitaire du travail entre les sexes. En cela elles se trouvent en décalage complet avec les nouvelles aspirations féminines.

L'égalité entre les sexes est-elle pour autant utopique? Nous avons vu qu'en Europe les procès de modernisation de la société vont dans le sens d'une égalité croissante. Encore faut-il ne pas sousestimer le rôle de l'environnement construit existant comme frein aux transformations sociales. Il importe de définir en architecture et en urbanisme des priorités tournées vers le futur, capables de contribuer à la requalification des espaces de vie.

Dans cette perspective, le fonctionnalisme unidimensionnel du logement de masse, la séparation systématique des fonctions urbaines et la division de la ville en zones unifonctionnelles se trouvent historiquement dépassés. Les préceptes légués par le Mouvement moderne doivent céder le pas à des concepts nouveaux: flexibilité, variété d'usages, planification ouverte avec participation démocratique de toutes les populations concernées représentent des concepts qui permettent d'intégrer de manière permanente les transformations techniques et sociales en cours. De manière complémentaire, les concepts de mixité de fonctions, d'imbrication des différents usages à l'échelle des quartiers et du bâtiment sont également à développer afin de rapprocher spatialement les différents domaines de la vie quotidienne, en particulier la sphère du travail et la sphère privée, et de créer ainsi une densité sociale et culturelle dans toutes les parties de la ville.

Du point de vue des femmes, l'objectif recherché est de favoriser, par la création d'un cadre architectural et urbain approprié, les processus d'émancipation en cours et à venir.

Références bibliographiques:

BARRIERE-MAURISSON M., 1992, La division familiale du travail, Paris, PUF, 251 p.

BECK-BERNSHEIM E., 1993, Das halbierte Leben. Männerwelt Beruf, Frauenwelt Familie, Frankfurt, Fisher Taschenbuch, 266 p.

BORST R., 1990, "Die zweite Hälfte der Stadt. Suburbanisierung, Gentrifizierung und frauenspezifische Lebensweiten", in R. Borst, S Kräfte, M. Mayer (Hrsg.), Das neue Gesicht der Städte, Basel/Boston/Berlin, Birkhäuser, pp. 235-268.

COMAILLE J., 1993, Les stratégies des femmes. Travail, famille et politique, Paris, La Découverte, 189 p.

DUBY G, PERROT M. (Hrsg.), 1994, Die Gescbichte der Franen, 5 Bd., Frankfurt/New York, Campus Verlag.

ELIAS N., 1969, Über den Prozess der Zivilisation, Bern, Verlag Francke AG (2e édition complétée). 
HERLYN U., v. SALDERN A., TESSIN W. (Hrsg.), 1987, Neubausiedlungen der 20er und 60er Jabre. Ein historisch-soziologischer Vergleich, Frankfurt, Campus, 315 p.

MARKUSEN A.R., 1980, "City Spatial Structure, Women's Household Work and National Urban Policy", Signs, No. 3, Vol. 5 Supplement, Chicago.

OCDE, 1985, L'intégration des femmes dans l'économie, Paris, OCDE, 201 p.

PARAVICINI U., 1990, Habitat au féminin, Lausanne, Presses polytechniques et universitaires romandes, $177 \mathrm{p}$.

PERRINJAQUET R., PARAVICINI U., 1993, "At Home in the City - Towards an Urban Periphery of the Future/Chez soi en ville - vers une périphérie urbaine du futur", Architecture \& Bebaviour (CH), Vol. 9, No. 3, September, pp. 285-311.

PERROT M., 1984, Une histoire des femmes est-elle possible?, Marseille, Paris, Rivages, 227 p.

SCHWARTZ-COWAN R., 1983, More Work for Mother. The Ironies of Household Technology from the Open Hearth to the Microwave, New York, Basic Books, 257 p.

SMITH N., WILLIAMS P., Gentrification of the City, Boston, 1987.

TAUT B., 1924, Die neue Wohnung - Die Fran als Schöpferin, Leipzig, Klinkhardt-Birmau.

WILSON E., 1993, Begegnung mit der Sphinx. Stadtleben, Chaos und Frauen, Basel/Berlin/Boston, Birkhäuser, 172 p. 


\title{
QUELLES VILLES POUR LES FEMMES \\ DANS LES PAYS DU SUD AU XXI ${ }^{\mathrm{e}}$ SIÈCLE?
}

\author{
FRANÇOISE LIEBERHERR-GARDIOL
}

Un colloque sur Femmes, villes, environnement! Est-ce pour jouer un effet de mode ou s'aligner sur une idéologie du «fémininement correct»? Ni l'un ni l'autre. Plutôt un engagement courageux pour un défi majeur à l'aube du XXI ${ }^{\mathrm{e}}$ siècle. Le monde de demain sera urbain avec probablement $50 \%$ de population urbaine en l'an 2000 et trois habitants sur cinq en ville en 2025 . Le monde de demain sera aussi masculin et féminin, c'est-à-dire qu'hommes et femmes s'engageront ensemble en partenaires responsables et solidaires à penser et décider comment résoudre les problèmes de leurs cités. Et le monde de demain devra être durable par un souci de gestion environnementale préservant les ressources et le cadre de vie. C'est une vision du XXI ${ }^{\text {e }}$ siècle incontournable. Le Rapport sur le développement humain 1995 vient de le rappeler: «e paradigme du développement durable doit intégrer pleinement la dimension féminine $»^{1}$

Dans la ville et l'environnement, les femmes ont subi une logique d'exclusion. Cependant, les années 90 ouvrent de nouvelles perspectives. Pour mieux comprendre les processus d'invisibilité des femmes dans les villes, jetons un très rapide regard historique sur le dernier demi-siècle dans les agglomérations urbaines du tiers-monde, où enjeux et rapports de force ont façonné le développement et la modernisation.

\section{LA MODERNISATION : LES FEMMES DANS DES VILLES MASCULINES}

La modernisation des villes apparaît comme un phénomène massif de la deuxième moitié du $\mathrm{XX}^{\mathrm{e}}$ siècle, où interagissent modèles de développement économique et paradigmes théoriques du développement. Si nous reprenons la classification de Caroline $\mathrm{Moser}^{2}$, on peut distinguer quatre périodes qui se succèdent dès les années 50. Dans la première période - «Modernisation et croissance des villes» - domine une pensée spatiale et physique. L'urbanisation se réalise par l'aménagement d'infrastructures variées en faveur d'une population perçue en moyennes statistiques et en projections démographiques. Domine aussi une norme essentiellement masculine telle qu'elle a été construite par l'histoire, faisant des femmes les oubliées des villes. Ainsi, la planification urbaine apparaît comme un domaine lié aux activités des hommes qui, à travers des disciplines dures telles l'ingénieurie ou l'architecture, organisent physiquement l'espace par les routes, maisons et infrastructures.

\footnotetext{
${ }^{1}$ Rapport sur le développement urbain 1995, New York, PNUD

${ }^{2}$ Moser Caroline, «Women, gender and urban development policy», Paris OECD Conference Women in the city, 1994 (paru aussi dans Lesfemmes et la ville, Paris, OCDE, 1995)
} 
A la fin des années 60, pauvreté et chômage se présentent comme des défis urbains majeurs. Il en résulte de nouveaux modèles de développement: «Redistribution de la croissance et besoins fondamentaux» qui caractérisent la deuxième période (1970-1980). Les analyses se concentrent sur le secteur informel et son importance critique comme source de revenus pour les femmes. Cellesci deviennent visibles dans les réseaux et les mécanismes de survie. Cependant, les politiques mises en œuvre pour combattre la pauvreté restent aveugles sur les contraintes spécifiques qui entravent le travail féminin, tels le manque d'accès au crédit, le travail sous-payé ou la mobilité restreinte.

Dans la troisième période 1980-1985, - «Lutte des femmes dans les villes» - des mouvements sociaux urbains de protestation contre l'insuffisance des services et des logements mettent en évidence l'importance de la participation des femmes à fournir des services de base pour les communautés et les familles, dans une gamme large qui couvre soins de santé et éducation, alimentation en eau, construction collective d'habitations ou organisation de bus. Emerge aussi la réalité d'autres modèles familiaux que la famille nucléaire stéréotypée, en particulier les ménages tenus par des femmes seules.

Depuis les années 85, la crise économique globale et les réformes macroéconomiques d'ajustement structurel, qui dominent le milieu urbain, caractérisent cette quatrième période comme «Crise des villes»: dérèglement général de la vie urbaine, inefficacité ou inexistence des services fournissant eau, électricité, transports, déficiences dans les besoins humains de base en santé et éducation, augmentation de la pauvreté, une situation d'ensemble qui appelle des solutions d'urgence. Filets de sécurité, mesures compensatoires, approches planificatrices transversales sont mis en place pour affronter des situations urbaines particulièrement complexes et en changement rapide. Mais dans l'ensemble, les mesures ne tiennent toujours pas compte des contraintes spécifiques des femmes: les femmes travaillent plus parce que les activités productives se cumulent au travail domestique sans allègement d'autres tâches, la distribution des ressources à l'intérieur de la famille ne se fait pas de manière égalitaire entre hommes et femmes comme l'avait laissé croire une hypothèse sur la répartition dans les ménages, et les tensions résultant de la crise économique augment la violence domestique, les maladies psychiques et la désintégration familiale.

\section{VERS UN NOUVEAU PARADIGME DU DÉVELOPPEMENT URBAIN}

Ainsi dans les années 90, la pensée urbanistique semble s'orienter vers une approche globale qui prend en compte le système urbain dans son ensemble. Il y a une décennie, les gouvernements percevaient encore très peu la globalisation et l'interconnexion des phénomènes concernant un environnement sans frontières, une mondialisation économique ainsi que la véritable participation et contribution des femmes au développement économique et social. En illustration, le dernier Rapport sur le développement humain 1995 en donne une estimation pertinente: «la production invisible des femmes, non traduite en valeur monétaire, équivaut à 11'000 milliards de dollars chaque année $\aleph^{3}$. Dans le courant actuel, le développement urbain s'ouvre à une approche complexe intégrant aux infrastructures physiques de base trois dimensions essentielles: sociale avec des équipements axés sur la santé, l'éducation et les services communautaires, économique avec des infrastructures pour la création d'emplois et de revenus, environnementale avec la lutte contre les dégradations écologiques et pour une gestion durable des ressources naturelles. Une manière de considérer que la ville n'est pas qu'une «affaire de logement, conduite d'eau et bus»,

\footnotetext{
${ }^{3}$ Op. cit., p. 1.
} 
mais aussi de groupes de quartiers, centres de santé et d'alphabétisation, associations économiques, recyclage des déchets, participation communautaire et pratique démocratique. C'est une vision holistique impérative pour promouvoir un développement humain durable centré sur les habitants des villes.

L'évolution des enjeux entre les deux Sommets sur les villes de Vancouver en 1976 et d'Istanbul en 1996 illustre la même tendance. Le concept d'habitat s'est transformé. Aujourd'hui, il intègre deux définitions en un tout: la première signification «d'établissements humains» adoptée il y a vingt ans et la signification d'écesystèmes» de Rio en 1992. Et parmi les six priorités du futur Sommet des villes figurent la gestion environnementale et la dimension féminine. Le secrétaire général d'Habitat, le Dr Wally N'Dow, a précisé: «On ne peut pas négocier le futur humain en l'absence de la moitié de l'humanité».4

Quant aux femmes, elles sont devenues plus visibles dans la ville, reconnues dans leurs multiples tâches productives, domestiques et communautaires, mais encore relativement exclues du monde des décideurs, des gestionnaires et des administrateurs urbains. Des rôles spécifiques utiles considérés comme féminins, mais pas encore des rôles de partenaires à côté des hommes œuvrant pour une cause commune. En effet, la perspective des rapports sociaux de genre s'est développée par un courant de recherche féministe, mais elle n'est encore guère sortie des milieux spécialisés. Lapproche de genre met en évidence que les différences entre hommes et femmes dans les multiples activités en société ne sont pas des données immuables liées à un déterminisme biologique, mais une construction sociale permanente des rôles et responsabilités féminins et masculins spécifiques, susceptibles d'évoluer et de changer. Cette notion centrale traverse toutes les cultures et tous les groupes sociaux, le masculin-féminin correspondant à une forme de partage des sociétés. Cette approche se situe aussi dans une critique sur l'universel masculin qui masque les différences femmes-hommes et qui est considéré communément comme un «ordre naturel» des choses, et sur une fausse neutralité du langage qui perpétue cet état de fait. C'est une façon nouvelle de questionner les rapports sociaux femmes-hommes, leurs complémentarités et leurs antagonismes. C'est aussi une perspective d'ouverture aux transformations possibles vers plus d'égalité et un véritable partenariat masculin-féminin.

\section{UN PROJET POSSIBLE: UNE CHARTE DES FEMMES DANS LA CITÉ}

En 2025, quatre milliards d'habitants pourraient vivre dans les villes des pays en développement. Ainsi, le projet urbain constitue l'un des enjeux majeurs du XXI ${ }^{\mathrm{e}}$ siècle; à ce titre, il doit concerner tous les habitants des cités, hommes et femmes, et les engager dans un processus démocratique de consultation et de décision. Les nouvelles idées ne manquent pas à l'aube du prochain Sommet des villes et la réflexion européenne pour une «Charte des femmes dans la cité» ${ }^{5}$ pourrait alimenter les débats autour des villes du Sud.

Cette charte vise à l'élaboration d'une nouvelle pensée de gestion urbaine, susceptible d'alimenter de façon constructive un véritable débat démocratique intégrant les besoins et les attentes diffé-

\footnotetext{
${ }^{4}$ Déclaration faite à la $4^{\mathrm{e}}$ Conférence mondiale des Nations unies sur les femmes de Beijing, septembre 1995.

${ }^{5}$ Charte européenne des femmes dans la cité, Bruxelles, Unité pour l'égalité des chances de la Commission des Communautés européennes, 1994.
} 
renciées des citoyens et des citoyennes. Comment progresser dans cette voie? Par des pas décisifs vers une citoyenneté active dans une société plus ouverte et libérée des stéréotypes d'infériorité qui condamnent les plus vulnérables, c'est-à-dire les pauvres et les femmes. Des pas importants seraient: favoriser le développement d'une autre philosophie de planification urbaine et de l'aménagement du territoire focalisée sur les valeurs humaines; susciter une nouvelle dynamique de responsabilisation sociale qui engage hommes et femmes à des décisions à la base; impulser une dynamique du changement bénéfique à l'ensemble d'une société devenue plurielle; enrichir la vision masculine par une perception féminine diversifiante et novatrice. Dans une optique globale de revitalisation des villes, il s'agit de reconstruire des lieux et des liens de cohésion sociale où les femmes auront un rôle de responsabilité et d'action.

Dans les contextes de crise, les femmes du Sud ont prouvé leur force et leur détermination. Les femmes seules responsables du ménage sont plus particulièrement touchées. Veuves, abandonnées, divorcées, elles deviennent toujours plus nombreuses: en moyenne, aujourd'hui dans les pays du Sud, les femmes ont la responsabilité d'une famille sur trois. Dans certaines villes d'Amérique latine, ces proportions s'élèvent à $50 \%$ des ménages. Partout la pauvreté se féminise, et pourtant les femmes plus que les hommes combattent la pauvreté quotidiennement. Elles assurent au jour le jour services gratuits et repas même les plus maigres à la famille, et dans le monde, elles sont responsables de la préparation de la nourriture à plus de $90 \%$. Selon différentes études, leurs revenus sont principalement consacrés aux besoins essentiels des enfants et de la famille, ce qui est moins le cas chez les hommes.

Ce sont les femmes aussi qui entreprennent des actions de lutte contre les dégradations environnementales. Elles sont au premier rang avec des idées innovatrices et elles multiplient les actions: ramassages des ordures, coopératives de recyclage des déchets, fours solaires, compostage, groupes de technologies alternatives, parmi d'autres. A l'échelle de la planète, elles ont apporté des solutions positives dans des situations négatives de crise. Elles se sont montrées partout des activistes convaincues et des actrices engagées dans des actions concrètes pour la préservation de l'environnement, parce qu'elles ont le sens du futur pour les générations dont elles se sentent responsables.

\section{LES FEMMES PORTEUSES D’AVENIR}

Le malaise urbain moderne qui a de nombreux visages: pauvreté, pollution, exclusion, mais aussi détérioration de la qualité de vie, déchirure des solidarités, déshumanisation des cités, appelle à l'évidence de nouvelles idées et de nouvelles personnes. Les femmes ont incontestablement un rôle à jouer: promouvoir une participation démocratique des femmes aux prises de décision et favoriser une réflexion sur les programmes d'aménagement à partir des lieux vécus quotidiennement pourraient y contribuer. Une manière de reconnaître que les femmes ne sont pas seulement des utilisatrices des villes, mais qu'elles peuvent concevoir, élaborer et décider de leurs espaces de vie. Les villes d'hier se sont construites sans les femmes. Les villes de demain devraient se construire avec les femmes.

Introduire les femmes dans la gestion urbaine devrait permettre de sortir des normes où l'universel est assimilé au masculin. En effet, une des caractéristiques de notre époque postmoderne est de reconnaitre la diversité des styles de vie et les variations d'âge, de genre, de culture, d'intérêt. Si le projet moderniste des dernières décennies a permis de grands progrès dans une certaine prospérité, un bien-être et des chances offertes aux individus, il a aussi imposé une rationalité économique et concurrentielle dominante qui laisse beaucoup d'exclus, de marginalisés. de pauvres. 
C'est pourquoi faire participer les femmes aux décisions dans la ville ne signifie pas simplement qu'elles doivent rejoindre les hommes dans leur pratique planificatrice, mais qu'elles vont contribuer à transformer le système. C'est un espoir de réaliser une participation démocratique pour une nouvelle gestion urbaine avec les femmes et les hommes qui vivent, utilisent et perçoivent les villes différemment ${ }^{6}$.

Le monde actuel en changement accéléré est marqué par l'incertitude. Comme les institutions et les idéologies qui ont guidé ce dernier demi-siècle sont devenues souvent inappropriées et que les aspirations se modifient rapidement, de nouveaux courants émergent. «Peu de choses sont plus significatives dans le monde que la possibilité d'un nouveau contrat social entre femmes et hommes, parce que la division de genre affecte toutes les autres stratifications dans les sociétés de

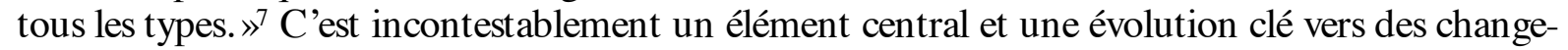
ments positifs dans les relations humaines.

\section{QUEL SCÉNARIO POUR LE FUTUR?}

Les scénarios pour le $\mathrm{XX}^{\mathrm{e}}$ siècle ne manquent pas, mais ils tendent à simplifier le possible: diaboliser les villes comme lieux de toutes les catastrophes ou les idéaliser dans une utopie de félicité et de bien-être. Ainsi s'opposent, stéréotypées, les villes du désespoir caractérisées par chômage, crime, pauvreté, pollution, services urbains dégradés et conflits sociaux, et les villes de l'espoir où dominent emplois, sécurité, prospérité, logements et services pour tous, centres éducatifs et culturels, démocratie participative.

De tout temps, les agglomérations urbaines ont été sources de progrès économique, social et culturel. Par un curieux paradoxe, les villes peuvent jouer des rôles inverses: diffuser créativité et innovation et sécréter en même temps pauvreté et misère. Il est important qu'au-delà de la crise actuelle, les villes retrouvent leur fonction positive comme moteur économique, lieu de communication, milieu créateur de culture, et espace ouvert à toutes les impulsions qui peuvent faire avancer l'histoire des humains. La responsabilité des femmes y sera engagée. Avec le défi de l'urbanisation rapide dans les pays du Sud, cela devrait être une évidence.

\footnotetext{
${ }^{6}$ Healey Patsy, «Integrating the concept of social diversity into public policy» Paris, OECD Conference Women in the city 1994 (paru aussi dans Lesfemmes et la ville, paris, OCDE, 1995)

${ }^{7}$ Giddens Anthony, Affluence, poverty and the idea of a post-scarcity society, Geneva, UNRISD, 1995.
} 


\title{
DU BON GENRE... OU DES RELATIONS DE GENRE APPROPRIEES
}

\author{
CHRISTINE VERSCHUUR
}

Il fut un temps où l'on cherchait dans la "technologie appropriée" un remède à certains problèmes du sous-développement. Malgré tout l'attrait que cette approche pouvait provoquer, une analyse attentive montrait que les effets cachés de cette démarche étaient de renforcer les rapports de production de type domestique et l'exploitation de ceux-ci. Cependant les technologies appropriées pouvaient favoriser les organisations de base et par ce biais introduire des changements sociaux.

Aujourd'hui plus que jamais, les sociétés se trouvent dans une impasse: dégradation de l'environnement, mondialisation d'un modèle économique de plus en plus inégal avec développement de sociétés à deux vitesses, et surtout absence de projets de société. Sera-ce en se retournant vers le "genre approprié" que nous trouverons une alternative à l'absence de perspectives pour les exclus et ceux qui rêvent d'une société plus juste et conviviale?

La femme incarne au mieux les charnières entre les tâches reproductives et les tâches productives, entre le temps libre ou le temps choisi et le temps nécessaire, entre ce qui a valeur d'usage et valeur marchande (il faudrait prendre au sérieux tout ce qui n'est pas estimable en prix d'achat ou de vente, le global par opposition au partiel). Les rapports de production de type domestique s'enchevêtrent avec ceux de type capitaliste. La surexploitation féminine que l'on observe actuellement et qui permet en grande mesure à l'économie mondiale de fonctionner résulte notamment des rapports entre ces deux sphères, la deuxième reposant de façon de plus en plus importante sur la première dans une situation de crise. Les tendances actuelles à privatiser les systèmes publics de prise en charge de la santé en sont une illustration.

Repenser, reconstruire le rapport entre ces deux sphères et entre les genres permettrait-il de bousculer la triste absence actuelle de perspectives de développement?

Les femmes entreprennent des projets alternatifs qui, s'ils forcent notre admiration et nous étonnent par leur créativité, ne doivent pas cacher le fait que cette "débrouille" est dictée par l'impératif de subvenir aux besoins du groupe familial, d'assurer la reproduction sociale dont elles se sentent seules responsables. Ces productions alternatives se réalisent généralement au coût d'une surexploitation du travail féminin. Les capacités de changements visibles dans ces initiatives féminines ne doivent pas faire oublier que les femmes restent absentes des lieux de pouvoir et de décision aussi bien au niveau des organisations de base lançant ces projets qu'à tous les autres niveaux. Mais les organisations de base ont un rôle fondamental à jouer en tant qu'espace de transformations et de luttes pour favoriser les changements de pouvoir entre hommes et femmes 
et rendre ses droits au genre "exproprié"... C'est en renforçant des organisations de base où se développerait une prise de conscience permettant de construire des relations de genre plus "appropriées" que l'on parviendra peut-être à sortir de l'impasse, à définir des projets de sociétés alternatifs pour un développement équitable et durable. 


\title{
PROCESSUS DE DECENTRALISATION MUNICIPALE ET ENVIRONNEMENT URBAIN: QUELLES PERSPECTIVES POUR LES FEMMES?
}

\author{
ISABELLE MILBERT
}

Les politiques de décentralisation, mises en oeuvre dans de nombreux pays en développement avec l'aide de différents bailleurs de fonds, répondent à deux objectifs principaux: d'une part, il s'agit de permettre un accès localisé à la démocratie - et de former les populations locales à celleci - et au fonctionnement du multi-partisme. D'autre part, sur le plan économique, on donne plus de responsabilités aux institutions locales, et les autorités recherchent une plus grande efficacité dans la délivrance des services urbains à l'ensemble de la population, y compris pour les groupes à bas revenus. Donc, dans de nombreux pays du Sud mais aussi du Nord, les années 80 et le début des années 90 ont été marquées par un important processus de décentralisation verticale, visant à déléguer certains des pouvoirs de l'Etat central aux niveaux inférieurs de l'Administration et à des structures représentatives des habitants et élues, c'est-à-dire aux provinces, municipalités, conseils, structures villageoises, et même parfois aux organisations de quartier, comme en Bolivie par exemple.

Sur le terrain, la mise en oeuvre de la décentralisation s'est avérée, dans les faits, plus difficile que prévu, et les lenteurs s'expliquent par la résistance des administrations centrales, la fragilité des démocraties et la faiblesse des délégations financières aux institutions locales. Cependant, chaque fois que ces politiques de décentralisation sont mises en oeuvre, on constate que les habitants des villes s'approprient très rapidement les éléments constitutifs du pouvoir local octroyé avec réticence.

Au coeur de ce processus se trouve d'abord l'enjeu de l'amélioration de l'environnement dans les quartiers pauvres. Une gestion plus locale offre-t-elle une approche plus participative et plus équilibrée de l'environnement? Permet-elle d'établir la liaison tant souhaitée entre les demandes des habitants et les investissements du gouvernement local?

Ensuite, dans le cadre de la décentralisation, les femmes apparaissent non seulement comme citoyennes électrices, mais aussi comme participant au premier chef à la gestion environ-nementale à la base, comme organisatrices de groupes d'action locaux, négociatrices avec les autorités, et, au terme de ce processus, comme actrices de la vie politique, citoyennes briguant un mandat. Peut-on donc affirmer, suivant cette logique, qu'une gestion plus locale des affaires publiques facilite l'accès des femmes à la responsabilité, à la décision et, finalement, au pouvoir politique? 


\section{UNE APPROCHE PLUS PARTICIPATIVE ET PLUS ÉQUILIBRÉE DE L'ENVIRON-NEMENT URBAIN}

Grâce à ce niveau de décision plus proche des habitants, on peut émettre quelques hypothèses:

- Les femmes pourraient, en tant qu'individus, faire connaitre leurs positions sur les problèmes de gestion quotidienne de la communauté. En effet, en tant que responsables de la santé quotidienne, de l'hygiène et de la gestion intérieure de la maison, elles sont les premières concernées par l'accès aux services essentiels. Leur approche est tournée vers les problèmes concrets constitutifs de l'environnement urbain. Une telle démarche rejoint les propositions de Jo Beall et Karen Lévy (1994) sur les impératifs de promotion de la "Gendered City".

- Par ailleurs, les femmes pourraient, en tant que membres des groupes d'action locaux, parvenir à un niveau de négociation avec les élus et avec la bureaucratie qui leur permette un meilleur accès à la prise de décision.

Cependant, même à ce niveau le plus local possible, qui implique surtout la communication, l'accès à l'information beaucoup plus que la prise du pouvoir, on constate d'immenses disparités: alors que certaines formes d'action sont devenues couramment le fait des femmes en Amérique latine (Moser, 1985), elles peuvent s'avérer très difficiles en Asie, où, même dans les programmes d'environnement urbain porteurs d'innovation et décidés à promouvoir la participation communautaire, la participation des femmes peut s'avérer très délicate.

Ainsi, dans le cas de Ghousia Colony, à Karachi, les travaux de Akerboom (1992) montrent que la majorité des femmes ont une opinion claire sur le programme d'assainissement et de réhabilitation, et sont tout à fait conscientes des avantages et désavantages que ce programme implique par rapport à leurs propres besoins en habitat. Par exemple, un certain nombre d'entre elles ont souligné l'inconvénient majeur de l'élargissement des ruelles à l'intérieur du quartier: les véhicules peuvent désormais passer et il est devenu dangereux pour les enfants de jouer dans la rue, laquelle perd son statut d'espace semi-privé pour devenir une voie de passage ouverte à d'autres que les riverains, ce qui présente de nombreux désavantages pour les femmes. Cependant, dans le déroulement du programme, il n'y a pas eu de prise en compte spécifique des remarques ou des besoins concernant les femmes. En fait, la plupart du temps, les hommes ont découragé toute participation aux réunions des femmes de leur famille, considérant cela comme indécent que "les femmes se mêlent des affaires des hommes". Certaines femmes interviewées manifestent une volonté de pouvoir intervenir, dans le futur, sur des sujets qui concernent leur maison et leur quartier, tandis que la plupart des autres adoptent une attitude beaucoup plus traditionnelle. De nombreuses femmes de ce quartier ne possèdent pas d'informations sur le processus électoral et ne manifestent pas le besoin de se regrouper en une association de femmes.

Bien que le cas du Pakistan apparaisse souvent extrême, il rappelle que tout progrès se situe encore dans un contexte de très grande vulnérabilité sociale des femmes: dans les quartiers pauvres de villes du Tiers Monde telles que Karachi ou New Delhi, les histoires de vie des femmes montrent toutes que leur position dépend très fortement de l'attitude et des opinions des membres masculins de la famille et de la communauté. De plus, les enfants apparaissent souvent comme des otages, la belle-famille menaçant de garder les enfants et de renvoyer la femme si celle-ci, par sa volonté de travailler, d'étudier ou de militer, sort par trop des normes correspondant au statut de la femme au foyer (Schuurmans, 1994: 27). Ainsi, dans la plupart des familles 
musulmanes, la position de relative liberté, d'accès au travail ou de participation à la prise de décision au sein de la famille et de la communauté, peut être remise en cause du jour au lendemain par un événement tel que le décès d'un père libéral ou le mariage d'une soeur à un époux traditionaliste.

Il reste que, dans les quartiers pauvres urbains, les femmes apparaissent, avec les enfants, comme les premières victimes de conditions environnementales extrêmement dégradées, qui additionnent les inconvénients courants en zone rurale (absence de réseaux, maladies traditionnelles, animaux vecteurs de maladies) et les problèmes typiques des villes (promiscuité, densité, pollution industrielle et par les transports urbains, accidents). Leur vulnérabilité est augmentée par le fait que, même si elles travaillent, elles passent chaque jour plus de temps que les hommes au sein du bidonville, alors que la plupart des hommes travaillent ou recherchent un emploi hors du quartier. D'autre part, elles sont confrontées directement au problème environnemental dans leurs tâches quotidiennes: trouver de l'eau propre, faire la cuisine au kérosène dans des huttes mal aérées, et bien pire encore, soigner les enfants et les membres de la famille victimes de ces conditions de vie dramatiques.

Ceci amène les femmes des quartiers à se mobiliser, mais en général sur des enjeux ponctuels. Ainsi, à New Delhi, dans le bidonville de Tigri, qui comprend 70'000 habitants (18'000 hommes, $12^{\prime} 000$ femmes et $40^{\prime} 000$ enfants vivant dans $10^{\prime} 000$ huttes), ce sont les femmes qui se battent pour la propreté d'immenses latrines collectives installées en un seul bloc au sein de ce quartier très dense, et pour la réparation des pompes manuelles qui tombent constamment en panne. "Mes deux bébés sont morts, déjà, à cause des mouches et de la saleté de cet endroit, disait l'une d'elles. Nous sommes allées en délégation à plusieurs reprises à la municipalité, sans succès." Dans ce cas, une femme leader, habitante du bidonville, parvient à canaliser la somme de ces souffrances pour faire pression sur les autorités et obtenir un certain nombre d'aménagements, ainsi qu'une mobilisation des habitants, hommes et femmes, autour d'un certain nombre d'enjeux: on note, dans ce quartier, la création d'une école par les habitants eux-mêmes ; l'organisation de ce groupe informel de femmes est reconnu par les autorités, et des bases associatives sont créées. Il convient de souligner le temps nécessaire et la complexité de la construction de ce genre d'organisation. Contrairement à ce que l'on pourrait croire, c'est en effet plus souvent la méfiance et la crainte mutuelle qui prédominent entre membres d'un même quartier, vivant dans une pauvreté et une précarité absolues.

Ce processus d'association et d'organisation de femmes d'un même quartier est exactement celui recherché par l'UNICEF et par la municipalité à travers la création de programmes d'actions privilégiant le rôle de la communauté, de "resident community volunteers" et, de façon centrale, de femmes "organisatrices de la communauté" (community organizers). Un point faible de ce programme, à notre sens, est le fait que les investissements sont extrêmement réduits, et il paraît difficile de maintenir un bon niveau de participation des habitants, sur le moyen terme, sans que des équipements minimums ne soient fournis.

Par ailleurs, dans cette démarche, les autorités indiennes aussi bien que l'UNICEF voient l'intervention d'ONG comme un appui ponctuel permettant d'augmenter le niveau des prestations dans des quartiers où tous les services manquent. Pourtant, nombreuses sont les ONG qui prennent aujourd'hui l'initiative d'impliquer les femmes dans leurs projets de santé, d'adduction d'eau, d'as-sainissement ou d'habitat. Leur engagement va de la fourniture directe des services à la communauté à un rôle de catalyseur entre les autorités et les communautés locales pour permettre une meilleure organisation des services. Pourtant, dans le cadre de ce projet UBSP (Urban Basic Services Program) qui s'est étendu à une majorité des quartiers pauvres des villes de l'Inde, les 
ONG ne sont pas placées au centre du processus. C'est la participation des femmes qui est recherchée.

Ainsi, pour la première fois en Inde, ce programme tente d'établir la liaison entre le processus d'auto-organisation des habitants des bidonvilles, la promotion de la femme et l'accès aux rouages du pouvoir décentralisé. Une telle démarche est sans doute très ambitieuse, mais, bien qu'il soit beaucoup trop tôt pour tirer des conclusions ou crier victoire, il semble qu'elle puisse être amenée à porter ses fruits lors des prochaines élections locales (1995-96), en particulier dans les Etats du Sud de l'Inde, le Karnataka et le Kerala: des sortes de "primaires" sont en effet organisées dans les quartiers pauvres pour installer sur les listes électorales des représentantes de la communauté qui ont activement participé depuis plusieurs années au programme d'accès aux services urbains de base.

Cet exemple nous paraît tout à fait exceptionnel, et le déroulement de ce processus électoral est suivi avec la plus grande attention. En effet, tous les progrès réalisés à la base, en particulier dans la prise en main de la gestion de l'environnement urbain, n'ont pas, pour l'instant, changé le paysage politique et l'équilibre entre hommes et femmes dans le processus de prise de décision, et ceci constitue l'interrogation centrale.

\section{DÉMOCRATIE LOCALE ET PARTICIPATION DES FEMMES}

Dans le cadre de la décentralisation, on peut imaginer que, puisque la collectivité locale est le premier échelon de la démocratie et que les femmes possèdent une solide réputation locale de militantes, de travailleuses sociales ou de leaders de groupes d'action de quartiers, elles pourraient ainsi se former à la vie politique et en gravir ensuite les différentes étapes.

Mais la réalité est bien différente: il y a bien, en effet, accès des populations pauvres à une plus grande démocratie et au processus électoral: on voit aujourd'hui, dans ces quartiers, hommes et femmes recevoir leurs cartes d'électeurs, qui sont souvent, pour les femmes, distribuées au porte à porte, alors que les hommes les reçoivent sur des stands où trône l'homme politique influent de la circonscription.

Les progrès sont donc réels, mais ils n'ont pas encore atteint, ou fort peu, la question des mandats électifs. Dans ce cas, dans toute l'Asie, les femmes sont toujours en position de minorité, et très souvent en situation de caution, captives d'un parti ou plus souvent encore d'un homme politique influent à qui, à tort ou à raison, elles attribuent leur ascension politique. Ce statut de minorité est conforté par le système des quotas, présents en Inde à tous les niveaux d'élection: or, un siège obtenu par quota est automatiquement déconsidéré.

En Inde, le nombre de femmes élues maires reste peu élevé, surtout dans les grandes villes (trois femmes élues maires depuis l'Indépendance), mais certains partis se sont fait une règle de désigner des femmes comme adjointes au maire. Cependant, cette fonction est purement honorifique et ne comporte un minimum de pouvoir qu'en cas de défaillance du premier magistrat.

Comme en Occident, au niveau d'une municipalité une bonne partie du processus de décision est 
initié à l'intérieur des commissions qui préparent les dossiers et les soumettent ensuite au vote du conseil municipal. Dans nos exemples indiens, il est alors intéressant de constater que, dans ces commissions, où il n'est plus question de quotas ou de réservations de sièges en faveur des femmes, leur proportion diminue très nettement, n'atteignant jamais plus de 3 sur 21, et souvent ces commissions ne comprennent pas de femmes du tout. A Bangalore, ville réputée libérale et moderniste, car elle est dotée de la plus importante communauté de cadres chercheurs et ingénieurs du pays, une seule femme a été amenée à présider une commission, en 1963; il s'agissait en l'occurrence de la commission sur l'éducation. Le plus fort pourcentage de femmes peut être trouvé dans les commissions concernant des domaines considérés comme "typiquement féminins", c'est-à-dire les services sociaux, la santé, et parfois l'éducation.

Au niveau local, exactement comme au niveau national, les femmes se voient conférer des portefeuilles correspondant au domaine traditionnel de compétence des femmes, c'est-à-dire principalement l'éducation, l'aide sociale et la santé. Ces tâches traditionnelles correspondent souvent, de la part des candidates, à un passé professionnel ou militant. En effet, on demande aux femmes une prise concrète sur les dossiers, une exigence de technicité, là où l'on ne demandera aux hommes que leur poids politique. Ces derniers reçoivent les portefeuilles qui constituent les enjeux essentiels de la décentralisation sur le plan politique et qui pèsent lourd sur le plan financier: l'ordre public, la police, l'eau et l'assainissement, les bâtiments.

Il est en fait rarissime qu'une femme parvienne, de la base, à grimper les échelons de la vie politique. En général, soit elle est politique et "première dame" d'emblée, pour des raisons familiales, d'équilibre régional, ethnique ou autre, soit elle est en position secondaire et y reste pendant fort longtemps.

L'exemple d'une enquête réalisée à Bangalore par K. Subha (1994) apparait ainsi très éclairant: $80 \%$ des conseillers municipaux viennent de familles qui n'avaient pas d'engagement politique particulier, et ils expriment le sentiment que, pour être un bon politicien, il n'est pas nécessaire d'appartenir à une famille engagée dans l'action publique. Par contre 76\% des élues soulignent l'engagement de membres de leur famille dans des activités publiques et le fait qu'elles ont grandi dans un environnement "éclairé" politiquement et socialement, ce qui les a influencées pour agir ensuite dans le domaine social.

Alors que l'on demande aux élues locales les raisons pour lesquelles elles ont gagné l'élection, presque la moitié d'entre elles (8 sur 17) répond que c'est à cause de leur travail social à l'intérieur de la communauté. Le fait qu'elles aient servi la communauté pendant de nombreuses années apparaît en effet comme un facteur de poids. Deux femmes attribuent leur élection au fait qu'elles ont été élues dans des quartiers où domine leur communauté d'origine, religieuse (musulmane) pour l'une, régionale (Tamoule) pour l'autre.

Une autre raison invoquée est la popularité du mari des conseillères municipales. A cause de la réglementation de réservation des sièges en faveur des femmes, les hommes, ne pouvant se présenter à l'élection dans cette circonscription, en confient la tâche à leur épouse, alors qu'ils disposent eux-mêmes de réseaux d'influence importants, souvent par le biais de leur activité politique ou de leurs activités sociales.

Aucune femme élue conseillère municipale n'invoque comme raison de son succès le fait qu'elle ait reçu l'appui des femmes de son quartier. Elle soutient au contraire que ce sont hommes et femmes ensemble qui participent à son élection. 
La totalité des femmes conseillères municipales sont membres d'une ou plusieurs associations touchant au travail social, et les trois quarts d'entre elles font partie d'organisations visant à résoudre des problèmes ayant trait à la vie quotidienne des familles, mais aussi aux tâches des femmes telles l'entretien des rues, la création de centres de santé et l'offre de nourriture non avariée.

En Asie comme en Amérique latine, on constate que, dans le cadre de la décentralisation, la coexistence entre le pouvoir local et les milieux associatifs des habitants n'est pas toujours facile, et l'accès aux nouveaux représentants de la municipalité n'est pas forcément aisé: d'une part, il y a souvent recrutement des éléments les plus représentatifs de milieux associatifs par les services municipaux eux-mêmes, ce qui peut aboutir à stériliser le militantisme et à transformer en rond de cuir un militant revendicatif; d'autre part, surtout dans le cas d'élections municipales en faveur de mouvements progressistes, le groupe d'action local peut se trouver pris à contre-pied par les politiques menées par la municipalité du même bord qu'eux.

Le dernier problème est sans doute le plus grave: les municipalités du Sud, où la collectivité locale manque en général des moyens financiers pour promouvoir l'environnement dans les parties de la ville qui en ont besoin, se trouvent dès leur élection en état de faillite virtuelle, ce qui les met en position terriblement défensive vis-à-vis des groupes d'action locaux et des femmes qui attendent des résultats concrets et rapides, encore plus que les hommes qui peuvent parfois se satisfaire pendant quelque temps d'une victoire politique. La décentralisation est souvent mise en oeuvre dans un contexte de faillite de l'Etat et de privatisation, ce qui condamne à moyen terme l'image de la démocratie locale, à moins que les municipalités ne réussissent à trouver d'autres formes de travail avec les habitants.

Il reste que les lois de décentralisation constituent une avancée juridique permettant aux groupes d'action locaux, y compris les femmes et les promoteurs de l'environnement de base, de peser plus directement sur la décision locale. Toute avancée juridique dans ce sens constitue, potentiellement, un énorme acquis. Elle s'inscrit alors dans une mouvance générale, au coeur de nombreux combats pour l'égalité et pour le partage de la prise de décision. Elle va de pair avec des avancées dans des domaines aussi sensibles pour la ville que son environnement et pour les femmes que la propriété, le foncier, l'héritage, la tutelle familiale, le droit de vote, le droit d'ester en justice et l'accès à la fonction publique.

\section{ELÉMENTS DE COMPARAISON}

Les exemples que nous avons choisis portent sur l'Inde et sur le Pakistan principalement, pays qui n'ont pas la réputation d'assurer un statut brillant à la femme, et qui, pourtant, ont placé à leur tête, pour des périodes longues, des femmes remarquables. Certes, aussi bien Indira Gandhi que Benazir Bhutto ont d'abord bénéficié du charisme et du soutien de leur père, et d'une éducation au sein de l'élite intellectuelle et politique internationale, mais elles ont l'une et l'autre été rélues sur leur propre image beaucoup plus que sur celle de leur famille, et leur longévité politique reste inégalée en Occident, mis à part Golda Meir et Margaret Thatcher. 
Dans les pays occidentaux, l'indéniable prise en main par les femmes de leur environnement, de leur travail, de leur vie personnelle, au cours des quarante dernières années, n'empêche pas qu'elle se trouvent face au "plafond de verre" au moment où il s'agit d'accéder aux rouages du pouvoir politique et donc à la prise de décision. En France, le nombre de femmes parlementaires est à peu près le même au dernières élections législatives de mars 1993 (35 femmes parlementaires, $6 \%$ des élus) qu'au moment où elles ont eu accès au vote et aux mandats électifs en 1945 (33 élues, 5,6\% des élus). Entre ces deux dates, une longue traversée du désert, entre 1958 et 1978, où les parlementaires femmes ne représentaient que 1,5 à $2 \%$ des élus! D'autre part, le pouvoir municipal des femmes françaises est particulièrement intéressant à analyser dans le détail: souvent élevée dans les organes périphériques, là encore la participation féminine va s'amenuiser à mesure que l'on se rapproche du centre politique, marquant encore une fois la division verticale du pouvoir entre les sexes: la part des femmes passe de $16,5 \%$ dans les postes de conseillers municipaux à moins de $6 \%$ dans les postes de maire. Encore s'agit-il, le plus souvent, de toutes petites communes rurales. En devenant maire de Strasbourg en 1989, Catherine Trautmann est la première à diriger une ville de plus de 100 '000 habitants. Notons néanmoins la meilleure performance des femmes dans la haute administration: $20 \%$ de femmes à la sortie de l'Ecole nationale d'Administration, 20 à $24 \%$ de femmes dans les cabinets ministériels français au cours de ces dernières années. Mais s'agit-il ici d'un réel accès à la prise de décision, ou de se retrouver dans la position si classique (et confortable?) de conseillère du Prince? La réalité oscille constamment entre ces deux interprétations.

De plus, exactement comme dans les pays du Sud, on observe, dans un contexte où l'Etat-providence est toujours puissant, la permanence de la vieille division entre le masculin politique et le féminin social. Les femmes qui accèdent à la scène politique se voient donc confier des mandats correspondant à des questions traditionnellement gérées par elles dans la famille: le social, la santé, le familial, le culturel... Cette division horizontale des rôles se retrouve à tous les niveaux de pouvoir et dans tous les pays, à l'exception de quelques pays scandinaves.

Le nombre de femmes maires constitue donc une infime minorité dans la plupart des pays occidentaux, alors qu'elles sont plus nombreuses comme adjointes au maire. En général, dans ces postes secondaires, elles jouent un rôle central dans la réélection de l'équipe, à cause de leur travail de fond opéré dans les quartiers, en particulier dans le secteur social (cf. Lyon et Bordeaux, au sein d'équipes qui se sont maintenues pendant 15 à 25 ans au pouvoir).

Le cas de l'Espagne, pays en transformation sociale rapide depuis 20 ans, mériterait de faire l'objet d'une analyse approfondie: en effet, il s'agit d'un pays aux rouages politiques et économiques très fortement décentralisés, où le pouvoir local est reconnu pleinement et s'affirme de façon militante. Or, la décentralisation ne semble en rien profiter aux femmes, comme si le local était plus réfractaire à l'image de la femme d'action que le gouvernement central: la participation des femmes dans les assemblées parlementaires (Chambre des Députés et Sénat) est en progression constante; en ce qui concerne la Chambre des Députés, elle est passée de $6 \%$ à $15 \%$ entre les élections de 1979 et celles de 1993. Les chiffres sont comparables en ce qui concerne les assemblées régionales. Par contre, on note la moindre performance des femmes lors des élections municipales, puisque le pourcentage de femmes maires oscille entre $8 \%$ dans des régions telles que le Pays Basque et Madrid, et 2,9\% en Cantabrique ou 1,6\% en Andalousie. 12,6\% des conseillers municipaux espagnols sont des femmes, ce qui laisse à penser qu'une relève est possible, mais qu'elle sera bien lente. Néanmoins, tout comme dans le Sud, la multiplication des femmes dans les équipes locales permet la naissance d'une solidarité et la prise de conscience de nouvelles priorités, d'impératifs de gestion différents, de la part des hommes également. 


\section{CONCLUSION}

Tout comme dans l'entreprise privée, les femmes viennent buter contre ce que le féminisme américain dans les années 80 a appelé le "plafond de verre"; un "glass ceiling" qui leur interdit virtuellement l'accès au cimes hiérarchiques du pouvoir réel. Alors que l'accès aux postes de responsabilité est devenu un phénomène de masse, la question reste posée de l'entrée dans les sphères du pouvoir politique. Une hypothèse est que ce domaine puisse être le prochain que les femmes vont investir, après que la génération précédente a conquis les sphères de responsabilité en une quarantaine d'années, au cours d'une ascension très rapide. On peut également s'interroger sur la réalité du désir des femmes à accéder à ces niveaux de pouvoir qui impliquent la solitude de l'ambition: combats, manoeuvres, mobilité, éloignement de la famille, et parfois détours au détriment de l'action concrète.

Bibliographie:

AKERBOOM A., 1992, Women of Ghousia Colony, Opinion and Action of Women during a Slum Upgrading Programme in Karachi, Urban Research Working Papers, $n^{\circ} 31$, Amsterdam, Free University.

BASU Aparna, 1990, From Silence to Activism, communication to the 11th European Conference on Modern South Asian Studies, Amsterdam, July.

BEALL Jo and LEVY Caren, 1994, Moving Towards the Gendered City, Overview Paper for Habitat II Process, Nairobi, UNCHS (Habitat).

BOSERUP Ester, 1989, Women's Role in Economic Development, London, Earthscan Publications.

CHHACHHI Amrita, 1990, Forced Identities: the State, Communalism, Fundamentalism and Women in India, communication to the 11th European Conference on Modern South Asian Studies, Amsterdam, July.

DANKELMAN I. and DANKELMAN J., 1988, Women and Environment in the Third World: Alliance for the Future, London, Earthscan Publications and IUCN.

GOTHOSKAR Sujata, 1992, Struggles of Women at Work, New Delhi, Vikas Publishing House.

HALIMI, Gisèle, (éd.), 1994, Femmes: moitié de la terre, moitié du pouvoir. Plaidoyer pour une démocratie paritaire, Paris, Gallimard.

JAGER Linda de, 1990, The Impact of Women's Action Forum on Pakistani Society, 1977-1985, communication to the 11th European Conference on Modern South Asian Studies, Amsterdam, July. 
MAHADEVAN K., (ed.), 1989, Women and Population Dynamics: Perspectives from Asian Countries, New Delhi, Sage.

Ministerio de Asuntos Sociales, 1995, Spanish Women on the Threshold of the 21st Century, Madrid.

MOSER C. O., 1985, Women's Needs in the Urban System: training strategies in gender aware planning, London, Development Planning Unit, University College.

PAPANEK H. and MINAUlT G. (eds.), 1982, Separate Worlds; Studies of Purdah in Asia, New Delhi, Chanakya Publications.

SCHUURMANS Akke, 1994, Women and Purdah, The Position of Women involved in a low cost sanita tion project in Quetta, Pakistan, Urban Research Working Papers n 36, Amsterdam, Free University.

SEHGAL N., 1995, Women, Housing and Human Settlements, New Delhi, Ess Publications.

SINEAU M., 1992, "Droit et démocratie", in Duby G., Perrot M., Histoire des femmes en Occident, Tome V, "le XX Siècle", sous la direction de F. Thébaud, Paris, Plon, pp. 471-498.

SUBHA K., 1994, Women in Local Governance, Jaipur, RBSA Pub-lishers.

THORBEK Suzanne, 1994, Gender and Slum Culture in Urban Asia, New Delhi, Vistaar Publications.

WEISS Anita M., 1990, Responding to the State. The Women's Movement in Pakistan, 1979-1989, communication to the 11th European Conference on Modern South Asian Studies, Amsterdam, July. 


\title{
MIGRER C'EST RESISTER: A PROPOS DES FEMMES EN MIGRATION
}

\author{
LAURENT MONNIER
}

Pour Gail Pheterson, les femmes qui migrent le font souvent en tant que groupe systématiquement exploité, dont les conditions économiques sont étroitement liées à l'oppression politique. Cette auteure définit l'oppression sexiste "comme étant l'exploitation par les hommes du travail privé et public des femmes et l'imposition de cette exploitation par la violence ou la menace de la violence de la part des hommes" (Pheterson, 1994: 59). Ainsi, comme la nature de l'oppression sexiste est associée à un mécanisme d'exploitation domestique, il est pertinent, du point de vue des femmes, de considérer que toutes les formes de migration sont des actes signifiants sur le plan politique.

\section{Thèse}

La réflexion de Gail Pheterson s'organise à partir d'un constat: sauf cas exceptionnel, affirme-telle, les gouvernements continuent à refuser de reconnaitre les persécutions fondées sur l'oppression de genre comme un motif légitime pour accorder l'asile, en dépit des prises de position favorables de certaines instances internationales, tels le Parlement européen ou le Comité exécutif du HCR.

Les femmes qui transgressent les codes discriminatoires en matière de genre, et celles qui s'émancipent, économiquement ou sexuellement, sont soumises à des sanctions sociales ou légales; elles ne peuvent espérer aucune protection. L'auteure donne de nombreux exemples où le stigmate lié à l'avortement, à la prostitution ou à l'homosexualité (alors qu'il s'agit pour les femmes de la liberté de disposer de leur corps) est suffisant pour les discriminer ou les criminaliser.

\section{Le statut d'infériorité des femmes}

\section{a) Pratiques sexistes}

En matière d'asile, on refuse aux femmes ce qu'on accorde aux hommes, car les pratiques administratives de la justice et de la police sont sexistes. Ainsi, en Allemagne, il est difficile pour une femme exilée d'être reconnue en tant que militante politique alors qu'on admettra sa qualité d'épouse de militant, comme si toute existence sociale autonome était déniée aux femmes.

\section{b) Pratiques discriminatoires}

Paradoxalement, la discrimation de genre conduit les fonctionnaires des pays occidentaux à rejeter les demandes d'asile de militantes parce que "pas assez politiques" alors que leurs activités dans 
leur pays d'origine sont considérées comme "trop politiques". L'auteure se réfère ici à des femmes du Kurdistan turc, faisant partie d'organisations nationalistes, qui ont été torturées et violées en prison par des militaires pour les punir de leur défaillance à l'égard de leur rôle traditionnel de femmes. Ces femmes, une fois libérées, sont rejetées par leur propre réseau partisan pour le même motif de transgression des impératifs de genre. Ensuite, quand ces mêmes femmes demandent l'asile dans des pays du Nord, elles se voient opposer une fin de non-recevoir, car le viol subi n'est pas considéré comme un cas de violence exceptionnelle et ne justifie donc pas l'octroi de l'asile.

\section{Migrer c'est résister}

La majorité des femmes qui cherchent à entrer dans un pays étranger ne le font pas pour fuir un système de persécution contre lequel elles se sont dressées. La plupart des migrants, hommes ou femmes, sont principalement en quête de travail et de revenus. Cependant, Gail Pheterson relève subtilement que les femmes qui migrent remettent en question plus ou moins implicitement les normes du contrôle patriarcal.

Etant donné le fondement économique - donc politique - de l'oppression de genre, il en découle que toute migration féminine a des implications politiques. Migrer est peut-être alors la forme la plus courante de résistance à une telle exploitation.

A l'échelle mondiale, l'augmentation des migrations féminines est l'amorce d'un processus de remise en question de la domination de genre: celles-ci représenteraient près de la moitié des migrants, alors que la population globale des réfugiés est composée à $75 \%$ de femmes et que plus de $60 \%$ des familles réfugiées ont à leur tête une femme.

Or, comme l'ont relevé Laura Oso et Christine Catarino, la migration féminine est un phénomène invisible. Cette "invisibilité de la migration féminine a été créée par le stéréotype de la femme considérée économiquement inactive et dépendante de l'homme" (Oso \& Catarino, 1994: 1).

La littérature courante et les statistiques déficientes contribuent à cette relégation des femmes dans des rôles passifs, alors qu'elles sont au contraire les véritables actrices de la migration, soit comme femmes qui restent, soit comme femmes qui partent (ibid.).

La thèse de Gail Pheterson met bien en évidence les mécanismes paradoxaux de l'oppression de genre, qui s'expriment "dans des situations de double contrainte inextricables".

Les femmes peuvent être punies pour être parties, alors que dans une large mesure ce départ leur a été souvent imposé. Or, l'autonomie que représentent pour des femmes le voyage ou l'initiative économique - que cette autonomie corresponde à des actes d'obéissance ou au contraire de résistance - implique la transgression des rôles féminins traditionnels. En effet, l'action initie le changement qui est la négation même de la passivité. Les réactions à cette autonomisation des femmes, perçue comme une transgression, tendent à les criminaliser, que cela soit dans leur propre pays, aux frontières ou dans le pays où elles se rendent.

Comme le dit l'auteure, "il y a de fortes chances pour que le stigmate et le chef d'accusation auxquels la femme migrante par besoin économique va être confrontée seront la prostitution" (Pheterson, 1994: 60). 
On pourrait donc articuler la réflexion de Gail Pheterson à des interprétations qui envisagent les migrations comme capables d'affaiblir le rôle traditionnel de l'Etat-nation (Badie \& Withol de Wenden, 1994: 11 ss). De nos jours, l'Etat n'est plus l'acteur unique et dominant des relations internationales. Le monde n'est plus exclusivement celui des Etats, car ce champ est investi de pratiques nouvelles associées aux notions de flux religieux et de flux migratoires impliquant d'autres formes originales de solidarité.

A un monde des Etats vient progressivement se superposer un système de réseaux, indépendant de l'espace territorial national. En d'autres mots, les mig rations remettent en question les règles traditionnelles de conduite inter-étatique.

En effet, par contraste avec l'Etat, sujet des relations internationales, "il est en revanche, pratiquement impossible de traiter avec des flux qui résultent de la seule agrégation de choix individuels ou, en tout cas, de micro-stratégies" (ibid., 13).

Il n'est donc pas indifférent de savoir que ces micro-stratégies sont principalement le fait de femmes. Dès lors, la tension entre logique d'Etat et logique migratoire, qui contribue à dramatiser voire diaboliser cette dernière, s'inscrit dans une perspective de lutte contre l'oppression de genre.

Ne plus miser sur l'Etat en appuyant ces formes de mouvements sociaux en gestation, c'est aussi contribuer à la visibilité des femmes comme nouvelles actrices urbaines qui préparent par un patient travail de fourmis un environnement libéré et solidaire où l'Etat-nation, symbole de la violence "machiste", sera définitivement évacué dans les poubelles de l'histoire.

\section{Références:}

BADIE Bertrand et WITHOL DE WENDEN Catherine (sous la direction de), Le défi migratoire, Paris, Presse de la Fondation nationale des sciences politiques, Questions de relations internationales, 1994, $185 \mathrm{p}$.

OSO Laura et CATARINO Christine, "Femmes-Chefs de ménage et migration", Au Nord et au Sud, les femmes du tiers monde face à la monoparentalité, Paris, Colloque international ORSTOM-AFED, 28-30 novembre 1994, 33 p.

PHETERSON Gail, "Droit d'asile, migration et prostitution", in Caloz-Tschopp Marie-Claire et al. (eds.), Asile, Violence, Exclusion en Europe. Histoire, analyse, prospective, Genève, Cahiers de la Section des Sciences de l'Education de l'Université de Genève et Groupe de Genève "Violence et droit d'asile en Europe", 1994, pp. 57-66. 


\title{
LA VILLE AU FEMININ ET AU MASCULIN
}

\author{
RODRIGO VIDAL ROJAS
}

L'histoire de l'urbain révèle de manière flagrante la permanence d'un rapport de domination de la femme par l'homme. A des degrés différents et dans des modalités qui varient selon l'époque et les cultures, les villes ont été construites et ont vécu jusqu'à aujourd'hui dans un rapport de domination et de soumission plus ou moins cruel pour les femmes. Cela reste un fait incontestable.

Pourtant, les explications données par les sciences sociales, et particulièrement par la sociologie, bien que clarificatrices quant aux mécanismes observables de cette domination, restent insuffisantes pour en expliquer les racines profondes. Cela, tout simplement, parce que ces racines trouvent leurs origines au coeur même des êtres humains. Ces origines, individuelles et collectives, sont explorées, parmi d'autres, par la psychologie, la mythologie et l'anthropologie culturelle.

L'intégration à l'analyse urbaine des concepts et des méthodes scientifiques, nouveaux ou déjà connus, mais qui n'ont pas encore été suffisamment exploités dans la recherche urbaine, peut constituer une véritable contribution au progrès de la connaissance scientifique afin d'expliquer la complexité de la ville en allant au-delà des approches urbaines traditionnelles.

Bien que la science ne soit pas cumulative, les concepts et les méthodes mis à jour par une discipline ne sont jamais perdus pour autant. Ils peuvent en tout temps être "re-visités" scientifiquement, c'est-à-dire actualisés et contextualisés.

A cet égard, l'approche junguienne n'a pas véritablement été l'objet d'une "re-visitation" par des disciplines scientifiques favorisant les études urbaines. Cela s'explique en partie par la faible importance attribuée, à tort, à la psychologie dans la compréhension de l'urbain. L'étude du phénomène urbain, objet par excellence de la sociologie, de l'histoire et de la mythologie collective, a quasiment négligé les racines psychologiques et spirituelles des compor-tements sociaux.

Ce texte n'est pas interdisciplinaire; il n'exclut pas pourtant des explications apportées par d'autres disciplines. Il constitue un effort scientifique pour mieux comprendre la dynamique urbaine dans la perspective du féminin et du masculin.

\section{LA POLARITÉ FÉMININ/MASCULIN EN PSYCHOLOGIE DES PROFONDEURS}

L'approche féminin/masculin nous permet, d'une part, de prendre comme point de départ de cette réflexion l'être humain lui-même en tant qu'objet et sujet de la production de la ville. 
D'autre part, cette approche, avec sa polarité paradoxale et complémentaire à l'intérieur de l'inconscient humain en psychologie des profondeurs, permet de dépasser le conflit culturel homme/femme et de le réinterpréter en le plaçant dans un rapport dialectique de complémentarité.

Selon C.G. Jung, "chaque homme porte en lui une femme"; il l'appellera anima. Il ajoute que l'animus, personnification masculine de l'inconscient chez la femme, se manifeste spontanément à travers les 'convictions' rigides et 'sacrées' ou l'obstination butée de la femme.

Nous pouvons ainsi préciser que l'animus correspond à la part psychique complémentaire masculine dans l'inconscient de la femme et que l'anima correspond à la part psychique complémentaire féminine dans l'inconscient de l'homme, selon la psychologie des profondeurs de Jung.

Toujours selon C.G.Jung, "l'inconscient (individuel) contient tout ce qui a été acquis au cours de l'existence personnelle... par conséquent, ce qui est oublié, refoulé, les perceptions subliminales, les pensées et les sensations"; l'auteur s'éloigne ainsi de la théorie freudienne qui "considère l'inconscient comme étant uniquement le lieu où sont déposées toutes les manifestations désagréables, indésirables ou inutilisables d'une manière quelconque" (Emma Jung et James Hillman, 1981: 65).

L'inconscient collectif est une instance de la psyché commune à tous les individus, faite de la stratification des expériences millénaires de l'humanité. Selon C.G. Jung: "L'être humain est en possession de bien des choses qu'il n'a jamais acquises par lui-même, mais qu'il a héritées de ses ancêtres. Il ne naît pas tabula rasa mais simplement inconscient... Les systèmes hérités correspondent aux situations humaines qui prévalent depuis les temps les plus anciens" (C.G. Jung, 1963: 230, 231).

Toujours selon C.G. Jung, les qualités féminines chez l'homme et masculines chez la femme sont tprésentes en chacun de nous mais ne peuvent pas toujours s'exprimer parce qu'elles gênent notre adaptation à notre milieu ou à l'idéal culturel établi.

Le second message central de la psychologie des profondeurs est que tout ce qui est inconscient en nous peut resurgir par des projections. Les mythes, les légendes, les contes que l'on trouve dans toutes les cultures et à toutes les époques sont "l'expression des réalités psychiques internes" en psychologie des profondeurs. L'analyse de ces représentations culturelles démontre de manière étonnante la présence de la figure de l'anima. Tous ces contes et légendes soulignent l'importance pour l'homme de donner une place au principe féminin qui fait aussi partie de son être.

Le refus ou la non-acceptation/assimilation de cette composante féminine chez l'homme provoque en lui un refus des projections externes de ce principe et donc le refus et le mépris des femmes, de la nature (mère-terre) et de la ville.

C.G. Jung estime que les figures de l'anima, chez l'homme, et de l'animus, chez la femme, "sont des dispositions à la relation avec l'autre sexe qui ont pris forme avec l'humanité elle-même" (Humbert, 1983: 63) et qu'il s'agit donc de dispositions innées chez les individus. 
Nous transposons nos conflits réels internes vers des situations, objets ou personnes réels externes. Les images que nous aurons de notre mère conditionnent nos rapports et nos images des autres femmes et de tout ce qui représente le féminin. Ces projections externes de nos images internes vont finalement marquer nos comportements sociaux et influencer nos perceptions cosmiques.

\section{LE FÉMININ/MASCULIN COMME FORME DE CONCEPTION ET DE PERCEPTION DES VILLES ANTIQUES}

Les historiens des villes ne sont pas toujours d'accord sur l'origine de la ville. Pour les uns, les cavernes pré-typifient la ville. Pour les autres, ce rôle annonciateur est incarné par l'arche de Noé. Cätal Hüyäk, dans l'ancienne Turquie, constitue encore le début de l'ère urbaine pour un bon nombre d'historiens. Il y a les contestataires qui lui préfèrent Ur des Chaldéens.

En ce qui concerne la ville occidentale, la Grèce, et notamment Athènes, est la référence obligatoire bien que Rome soit encore considérée comme le berceau de notre civilisation urbaine.

Pour notre réflexion, l'origine exacte de la civilisation urbaine reste secondaire. Quoi qu'il en soit, l'essentiel est de dévoiler les formes et les récits de cette origine. Un récit est une relation, une narration, une histoire, une fable d'événements réels ou imaginaires dont peu importe la véracité historique. Son but est de nous informer sur les liens (relations) existants entre un acte accompli et l'idée fondatrice de cet acte. Un récit est un enjeu de valeurs et normalise le passé.

L'histoire cherche l'objectivité scientifique dans la relation des faits cités et c'est la raison pour laquelle elle est l'objet d'une mise en question permanente. Le récit, lui, n'est jamais vrai ou faux et son importance, pour notre analyse, tient au fait qu'il n'exprime pas nécessairement les événements qui se sont succédé au cours de l'histoire, mais, et surtout, la manière dont les individus pensent ou veulent que les choses eussent pu se passer. C'est une idéalisation historique.

Les récits de fondation de villes se trouvent à mi-chemin entre l'histoire et la mythologie. Une de leurs caractéristiques essentielles est qu'ils sont post-factum; ils apparaissent après la fondation des villes afin d'expliquer cette fondation. Certains récits nous permettent, d'un côté, de connaitre l'image qu'un groupe social, à un moment donné de son histoire, a de lui-même et la manière dont il conçoit sa reproduction. D'un autre côté, si l'on s'en tient à l'idée selon laquelle l'organisation de l'espace est un reflet de l'organisation d'une société, ces récits reflètent l'image-type que ce groupe se faisait du lieu où il devait résider.

"Aussi la fondation d'une ville était-elle toujours un acte religieux" dans l'Antiquité (Fustel de Coulanges, 1984: 151). La ville constituait le sanctuaire majeur de la réunion éternelle des hommes et de leurs dieux (croyances) sur un même site. Aux origines, un foyer central (une fosse où chacun avait déposé un peu de terre de son lieu d'origine dans la tradition romaine), une enceinte sacrée et, plus tard, les murailles, les portes et le pomoerium constituent la ville. 
La plupart des récits insistent sur les rapports conflictuels entre la ville et son territoire. Dans la tradition étrusque, la cérémonie de fondation d'une ville commençait par la constitution du foyer central autour duquel devait s'élever la ville. Le prêtre, la tête voilée et revêtu du costume sacerdotal, traçait le sillon sacré, enceinte symbolique de la ville qui devient inviolable. Pour ce faire, il se servait d'une charrue tirée par un taureau blanc et une vache blanche. Le prêtre tenait luimême le manche de la charrue qu'il portait à certains intervalles afin d'interrompre le sillon et de créer des portes. Les mottes de terre (sacrée) soulevées par la charrue étaient jetées soigneusement à l'intérieur de l'enceinte. Lorsqu'il avait fini de tracer le contour de la ville, la vache restait à l'intérieur du sillon et le taureau à l'extérieur. Cette enceinte était sacrée; la toucher ou la traverser était considéré comme un sacrilège, un acte d'impiété.

Dans cette procédure d'individualisation d'un lieu sur un territoire, la vache représentait dans l'imaginaire des fondateurs le principe féminin, le taureau symbolisant le principe masculin. L'enceinte créait un intérieur et un extérieur, un familier et un étranger, un local et un global, un connu et un inconnu.

Cet intérieur, familier, local, connu, est sacré et inviolable. C'est là que règne le principe féminin, lequel cherche à "unifier et à unir", comme l'anima chez C.G. Jung (cité par Humbert: 66). Cet extérieur, étranger, global et inconnu, c'est le territoire à conquérir et à dominer. Ici règne le principe masculin ou animus qui cherche à "distinguer et à connaitre" (ibid.). L'impureté habite l'extérieur, la pureté sacrée, l'intérieur.

L'étude de la cité de Catal Hüyäk, en Turquie méridionale, riche en images, montre comment dans d'autres cultures et civilisations le féminin organise symboliquement, spatialement et fonction-nellement les lieux. Ici, le féminin s'exprime à travers l'image de la femme et la présence de la mère-terre. La figure de la femme, symbole de fécondité et de reproduction de la vie, apparaît sous forme d'un corps de déesse, bras et jambes levés en signe d'accouchement, et à travers des images de seins.

"La place du mâle est tenue par des taureaux et des béliers, expressions les plus fortes de la virilité" (Mellart, 1971: 101). Mais le taureau et le boeuf représentent aussi le fort attachement et la dépendance vis-à-vis de la mère-terre. Il est le premier animal apprivoisé pour le travail, notamment pour les activités agricoles dont dépendaient les habitants. En effet, ces derniers avaient de grandes connaissances en agriculture et une bonne technique pour conserver efficacement les aliments.

Mais la terre était aussi leur foyer. Catal Hüyäk est une ville souterraine située dans une colline. Elle existe par et dans la terre. Leur rapport à celle-ci ainsi que leur attachement était si grand que les habitants de Catal Hüyäk enterraient leurs morts dans le sol de leurs maisons.

Dans cette ville il n'y a ni places, ni parcs, ni rues, toutes les maisons étant collées les unes aux autres. L'on sort par des trous pratiqués dans le plafond. L'intérieur des maisons est multifonctionnel: c'est le lieu sacré, le lieu de toutes les activités quotidiennes et du repos éternel. Tout se réalise à l'intérieur, à l'exception des activités de subsistance. L'extérieur est monofonctionnel: c'est le lieu du travail agricole et, dans une moindre mesure, de la chasse d'animaux.

La reproduction est le fruit du sacré familial, intérieur. La préservation est le fruit du travail extérieur. 
Dans beaucoup de récits, la fondation d'une ville est attribuée à une déesse ou à une femme qui deviendra une déesse après la fondation. C'est le cas pour la ville sumérienne d'Akkad dont l'instauration a été prise en charge par la déesse Inanna ou encore pour la grande Carthage dont la fondatrice est Elyssa, vierge de grande beauté, soeur de Pygmalion, roi des Tyriens.

La ville est très souvent associée à la maison, lieu sacré du lien entre les dieux et les hommes. En Mésopotamie, et notamment à Babylone, les ziggourats représentent le trait d'union entre la terre et le ciel. En construisant la tour de Babel, les hommes ont voulu "toucher le ciel" et sortir du cadre terrestre de la ville: le ciel est le sacré suprême. Cela ne leur a pas été permis. C'était l'utopie masculine qui voulait s'imposer au possible féminin du sacré sur terre.

"Militaire ou religieuse, administrative ou marchande, la ville antique est avant tout imprégnée de religiosité, et le sacré imbibe chaque brique de chaque maison, chaque pierre de chaque route" (Paquot, 1990: 23).

Les protagonistes de ces oeuvres étaient-ils conscients de ces symboles et significations? Assurément non. D'abord, pour une raison historique. Un grand nombre de ces significations nous sont dévoilées grâce à la mise en perspective historique (le recul du temps) et aux possibilités actuelles de comparer des situations historiques, possibilités dont les acteurs ne disposaient pas.

Ensuite, pour une raison psychologique. Humbert, en paraphrasant C.G. Jung, explique que "un symbole est (donc) une expérience... le symbole est vivant... D'une façon générale, l'action du symbole est celle d'une représentation qui engendre un sens parce qu'elle fait se rejoindre des termes séparés. Le fait de sens qui accompagne une telle expérience s'impose et, cependant, il échappe à la raison... le symbole est caractérisé, en effet, par un certain rapport à l'inconnu" (Humbert, 1983: 43, 44).

Les peuples anciens avaient ainsi créé des images dont ils n'étaient pas toujours conscients. Aujourd'hui nous interprétons ces images et ces symboles et, bien qu'une bonne partie de leur sens nous échappe, ils nous permettent de saisir le mystère psychologique de la projection sur la réalité externe d'une réalité consciente/in-consciente interne. 


\section{LES CATÉGORIES DU FÉMININ/MASCULIN COMME INTERPRÉTATION}

DE LA DUALITÉ VILLE/TERRITOIRE

Ces récits nous permettent de construire une première catégorisation que nous pouvons par la suite retrouver dans n'importe quel récit:

$\begin{array}{ll}\text { Féminin } & \text { Masculin } \\ \text { Ville } & \text { Territoire } \\ \text { Maison } & \text { Site } \\ \text { Local } & \text { Global } \\ \text { Connu } & \text { Inconnu } \\ \text { Intérieur } & \text { Extérieur } \\ \text { Prochain } & \text { Lointain } \\ \text { Fini } & \text { Infini } \\ & \\ \text { Multifonctionnalité } & \text { Monofonctionnalité } \\ \text { Unir } & \text { Distinguer } \\ & \\ \text { Réel } & \text { Utopique } \\ \text { Possible } & \text { Souhaitable } \\ \text { Mesuré } & \text { Mégalo } \\ \text { Reproduction } & \text { Préservation } \\ \text { Sacré } & \text { Profane } \\ \text { Familier } & \text { Etranger } \\ \end{array}$

Aujourd'hui, dans l'ethnie Toucouleur qui appartient à la communauté linguistique Hal Pulaar, au Sénégal et au Mali, la Djom Soudou est la femme propriétaire de la chambre. Le Djom Gallé est l'homme propriétaire de la maison et de son enclos. Ainsi, avec des siècles de distance et dans des espaces géographiques et culturels différents, nous retrouvons les mêmes catégories intérieur/extérieur, maison/site, ville/territoire.

Le symbole phallique, premier élément de structuration spatiale du territoire (menhir) traduit la domination de l'homme sur la femme et sur la mère-terre; c'est le phallocentrisme ou la primauté du mâle. C'est aussi une volonté de domination sur les forces de la nature (parmi lesquelles, les animaux). 
Cette domination signifie contrôle. Cet imaginaire explique en partie le rôle assigné au mâle et à la femelle dans l'organisation sociale. Ainsi, la femme c'est le local/localisé, du latin locus, qui est aussi la racine latine de lieu, d'une partie déterminée de l'espace, un lieu précis. Le local est un lieu connu. La femelle reste dans un lieu connu, elle est locale. Le mâle, c'est le global, du latin globus la terre, le globe.

Les récits de fondation reprennent cet imaginaire du féminin/masculin, local/global, passivité/activité dans la pro-duction de la ville. De cette manière, dans l'imaginaire ancien, le rapport féminin/masculin définit le rapport ville/territoire dans des sphères bien précises.

Le mâle/masculin est monofonctionnel. Il sort soit pour chasser, soit pour faire la guerre, soit pour travailler, soit pour les trois choses, mais jamais en même temps. La division des fonctions dans les villes, à différentes époques, est masculine: prêtre, militaire, commerçant, producteur, politicien, etc. Le masculin/mâle c'est la spécialisation. Le territoire est divisé avant d'être exploité afin de lui assigner des rôles différents selon sa morpho-géologie.

La femelle/féminin est multifonctionnelle. Elle reste pour nourrir, élever, protéger, guérir, nettoyer, attendre, organiser, etc., tout en même temps. Chez elle, il n'y a pas de spécialisation. La première définition de la ville découle de son caractère principalement multifonctionnel: lieu de relation, d'échange et d'information multiple.

Selon Riccardo Mariani, au Moyen Age, la ville naît en Europe lorsqu'une multiplicité de fonctions ne sont plus exercées par l'Eglise mais autour d'elle. L'Eglise se reproduit et engendre la ville, mais sans éparpillement, comme les poussins autour de la poule. Le village devient ville.

L'intérieur est la ville, le féminin dans les anciens récits de fondation. L'extérieur est le territoire, le masculin selon ces récits. L'extériorité et la globalité du territoire, par rapport à l'intériorité et la localité de la ville, sont idéalisées dans ces récits et elles expriment le besoin de l'homme d'extérioriser son principe féminin.

Relevons aussi que le souhaitable appartient au principe masculin tout comme le possible appartient au principe féminin. On entre ainsi dans l'idée de l'utopie.

L'utopie (du latin $o u=$ non; topos=lieu) c'est le non-lieu. L'utopie est une construction rigoureuse et imaginaire de la société. Toute rigueur renvoie à une méthode, à une manière de faire. L'utopie est la capacité de construire mentalement sur le néant, à partir du néant, du non-lieu. Le possible est raisonnable (non pas rationnel, même si les deux mots ont la même racine étymologique, ratio $=$ raison). Le raisonnable c'est agir conformément au bon sens, à partir d'une connaissance empirique. Le rationnel c'est déduire par le raisonnement et cela n'a rien d'empirique, ne dépend pas de la connaissance pratique du réel.

La Charte d'Athènes a voulu universaliser le masculin/utopique interne dans l'inconscient collectif. Elle a voulu tout mettre en question et recommencer à zéro en faisant table rase de l'existant, une nouvelle création. Ce n'est pas possible. Chaque culture doit en fait s'exprimer, extérioriser de manière spécifique cette polarité paradoxale et complémentaire: utopie/réalité, raison/émotion, souhaitable/possible. 
Les Plans directeurs d'urbanisme, tout comme les récits, expriment post-factum l'idéal de ville à construire, la ville souhaitable; une ville imaginaire conçue sur la table à dessin, hélas trop éloignée de notre malheureuse expérience quotidienne de la ville.

La créativité n'est pas l'imagination effrénée, ni l'aménagement conformiste, mais l'effort de permettre au souhaitable de devenir possible et non l'imposition du possible comme souhaitable. Un des principaux problèmes de la planification urbaine et de la planification tout court est que l'on a voulu organiser le souhaitable sans considération du possible (féminin). En revanche, le pragmatisme actuel veut imposer le possible en négligeant le souhaitable, l'utopique.

L'objectif est d'arriver à trouver une complémentarité accrue entre l'utopique, le rationnel, l'intellectuel et le souhaitable du masculin avec le réaliste, le sentimental, l'émotionnel et le possible du féminin.

\author{
IMPLICATIONS DE L'APPROCHE FÉMININ/MASCULIN \\ DANS LES RAPPORTS HOMME/FEMME EN MILIEU URBAIN
}

Au cours de notre histoire, nous avons à tort associé le principe féminin aux femmes et le principe masculin aux hommes. Il nous parait ainsi impensable de voir une femme prenant la tête du bureau de planification territoriale d'une mégapole latino-américaine. Cette association est erronée.

Le problème est que, en transposant les catégories du féminin et du masculin aux femmes et aux hommes, nous avons créé une nouvelle catégorisation:

$\begin{array}{ll}\text { Féminin } & \text { Masculin } \\ \text { Femme } & \text { Homme } \\ \text { Le foyer } & \text { La politique } \\ \text { La famille } & \text { La société } \\ \text { Elever les enfants } & \text { Faire la guerre } \\ \text { Reproduire } & \text { Conquérir } \\ \text { Rester } & \text { Partir } \\ \text { La communauté } & \text { La société } \\ \text { Le quartier } & \text { La ville-territoire }\end{array}$

Le féminin n'est pas la femme, le masculin n'est pas l'homme. La vie repose sur l'équilibre harmonieux des énergies masculines et féminines présentes dans l'action et dans l'inconscient de chaque être humain. La ville d'aujourd'hui exprime une rupture de cet équilibre (écologique, sociologique, biologique, psychologique et mythologique). 
La ville actuelle, en étendant sans cesse son territoire, afin de le maittriser et de le soumettre à travers un processus couramment appelé "mégapolisation", a généré une dynamique inverse: elle a été modifiée dans sa structure profonde et a provoqué une énorme crise dans son fonctionnement, dans sa structure et dans son écologie.

Le défi écologique, la protection de la mère-terre, est un défi féminin qui doit encore s'ouvrir un chemin à travers la méfiance de la masculinité du savoir scientifique.

Un des grands obstacles à surmonter aujourd'hui est lié à l'image de la ville. La mégapolisation a produit une rupture de la frontière ville-territoire. Le principe masculin exprimé dans le désir de domination, de conquête, de mégalomanie a écrasé le principe féminin de maitrise intérieure, de mesure, de contrôle des frontières du réel.

En se mégapolisant, la ville dévore le territoire, l'urbain envahit la société et les efforts de décentralisation territoriale et de maitrise de la croissance des villes se heurtent à un nouveau paradigme: la défiguration du local et du global. Le local se relocalise à l'intérieur d'une ville atomisée qui devient le global. La ville s'étend et devient territoire, ville diffuse. Le territoire s'urbanise. La ville-féminine se territorialise et donc se masculinise.

Ainsi, la structure territoriale actuelle de la ville, atomisée, multilocalisée, fragmentée, empêche l'expression des valeurs féminines du local, connu, intérieur, mesuré. Avec la mégapolisation et la globalisation, la ville devient ouverte et perméable. La globalisation a cassé l'intérieur, l'intime, le prochain, elle a violé et traversé l'enceinte sacrée du local et profané la ville.

L'étranger devient local, le familier global. Le prochain est profané, le lointain sacralisé. L'association erronée du féminin avec les femmes conduit ces dernières à un confinement vers un rôle jugé péjorativement comme secondaire. Par cette même association erronée, les hommes s'érigent une fois de plus en maîtres de cette violation du sacré intérieur de la ville. Ce sont principalement eux qui construisent une Europe de villes ouvertes et défigurées, centraliste et étrangère aux intérêts des citadins.

En assumant son inconscient masculin, nombre de femmes latino-américaines construisent avec créativité le souhaitable à partir du possible et nourrissent leurs familles dans des contextes de crise économique. En assumant leur inconscient féminin, des hommes de partout reconnaissent l'importance vitale de la protection de la mère-terre souffrante et agonisante et s'engagent dans sa préservation.

Mais en même temps, des millions de femmes en refoulant leur inconscient masculin renoncent à lutter pour conquérir un rôle complémentaire des hommes qui leur permette de sortir de la soumission actuelle. De même, en refoulant leur inconscient féminin, des millions d'hommes continuent à détruire la mère-terre, à profaner la ville par la mise en place de politiques qui n'empêchent pas sa défiguration territoriale ni l'exploitation et la domination des femmes.

En conclusion, la question que l'on peut se poser est: Comment faire en sorte que notre conception de la ville et la ville elle-même devienne plus humaine, c'est-à-dire intégrative de la totalité de l'être humain dans ses composantes féminines et masculines? 
Bibliographie:

AA.VV., 1983, La ville dans le proche-orient ancien, Leuven, Peeters, (Les Cahiers du CEPOA, Université de Genève, Actes du Colloque de Cartigny-1979), 310 p.

ANTOLINI André et BONELLO Yves-Henri, 1994, Les villes du désir, Paris, Galilée (Coll. Débats), $170 \mathrm{p}$.

BENEVOLO Leonardo, 1983, Histoire de la ville, Paris, Parenthèses (pour la version française).

COUSINEAU BRUTSCHE Diane, 1993, Le paradoxe de l'âme: exil et retour d'un archétype, Genève, Georg éditeur (Coll. "Etudes jungiennes"), 161 p.

DAGHINI Giairo, 1994, "Les mots de la ville", Les Cabiers de Philosophie, n 17, "Le philosophe dans la cité", hiver 1993/1994, pp. 25-33.

FOUSTEL de COULANGES, 1984, La cité antique, Paris, Flammarion, 494 p.

HUMBERT Elie G., 1983, C.G. Jung, Paris, Editions universitaires (Coll. Agora; Presse Pocket; 85), $161 \mathrm{p}$

JUNG Carl Gustav, 1963, Psychologie et éducation, Paris, Bouchet-Chastel.

JUNG Emma et HILLMAN James, 1981, Anima et Animus, Paris, Seghers (Coll. "L'esprit jungien" - traduction française), $221 \mathrm{p}$.

LE CORBUSIER, 1957, La Charte d'Athènes, Paris, Minuit (Coll. Points ; 25), 190 p.

MELLART James, 1971, Catal Hüyäk: une des premières cités du monde, Suisse, Jules Tallandier (pour la version française), $232 \mathrm{p}$.

PAQUOT Thierry, 1990, Homo Urbanus: essai sur l'urbanisation du monde et des moeurs, Paris, Edition du Félin (Coll. Essai), 178 p.

RACINE Jean-Bernard, 1993, La ville entre Dieu et les hommes, Genève, Presses Bibliques et Universitaires, $354 \mathrm{p}$.

RONCAYOLO Marcel et PAQUOT Thierry (sous la direction de), 1992, Villes et civilisation urbai ne: XVIIIe - XXe siècle, Paris, Larousse (Coll. Textes essentiels), 688 p.

RONCAYOLO Marcel, 1993, La ville et ses territoires, Paris, Gallimard (Coll. Folio/essais: 139 nouvelle édition), $278 \mathrm{p}$. 


\title{
UNE NOUVELLE APPROCHE DU PARTAGE DE L'EMPLOI: LE ROLE DES FEMMES
}

\author{
ANDRÀS NOVEMBER
}

Avec l'installation durable du chômage et de l'exclusion, le "partage du (temps de) travail" est devenu un enjeu socio-politique considérable. Le débat concernant le partage de l'emploi ne se limite pas à la tentative d'apporter une solution au chômage; il touche, en réalité, aux principes fondamentaux de l'organisation sociale. L'interrogation sur le rôle et le statut des femmes dans ce processus s'inscrit dans cette perspective. Or, le "facteur femme" est singulièrement absent des propositions. Le chômage et le partage de l'emploi sont abordés exclusivement sous l'angle "masculin" et pratiquement jamais au "féminin"1

\section{DE L'EMPLOI AU MASCULIN ET DU TRAVAIL AU FÉMININ}

Il convient de souligner d'emblée que "travail" et "emploi" ne sont pas des notions équivalentes; il ne faut pas les confondre, même si dans le langage courant les deux termes sont utilisés comme des synonymes. L'emploi correspond à une occupation rémunérée qui fait partie de la sphère écono mique. Le travail, en revanche, relève de la sphère sociale et n'est pas nécessairement rétribué. C'est le cas du travail domestique, accompli essentiellement par les femmes.

Avec l'avènement de la société industrielle, l'emploi salarié a progressivement remplacé le travail - dont l'utilité est à la fois économique et sociale - pour devenir le fondement même de la socialisation et de l'insertion des individus dans la société. Le travail non rémunéré, quant à lui, a été purement et simplement évacué du champ économique².

L'écart sémantique entre emploi (marchand) et travail (non marchand) se double d'un autre décalage, celui qui existe entre les valeurs "masculines" et "féminines" attribuées au travail. Cette rupture est illustrée par une étude menée dans les années 30 par Alfred Lotka, démographe américain bien connu. Il a voulu savoir quelle pouvait être la valeur apportée à la collectivité par un groupe, en calculant la somme de revenus accumulée par une cohorte de mille personnes au cours d'une $v^{3} e^{3}$. Le résultat de son enquête est édifiant: la valeur créée par les hommes salariés durant leur vie

1. L'absence du rôle des femmes dans le débat concernant le partage du temps de travail est une observation empirique que l'on peut certes contester. Je n'ai toutefois pas connaissance de contributions qui concernent spécifiquement les femmes quand il s'agit de partage de l'emploi.

2. Il existe pourtant de nombreux domaines où le travail n'est pas rémunéré par un salaire, comme le travail domestique ou l'éducation des enfants qui sont généralement pris en charge par les femmes. Il est vrai que le nombre des "hommes au foyer" est en augmentation, surtout parmi les jeunes. Mais ils sont encore déconsidérés.

3. Le calcul tient compte des revenus cumulés pendant la vie active d'une personne, en soustrayant les coûts occasionnés par son éducation et sa retraite. Cf. JACQUARD Albert, J'accuse l'économie triomphante, Paris, Calmann-Levy, 1995, p. 147. 
monte jusqu'à soixante mille dollars (au prix des années 1935-1940), alors que celle correspondant au travail des femmes est estimée à zéro dollar (sic!). Il n'est pas sans intérêt de se demander comment une étude "scientifique" peut aboutir à une conclusion aussi absurde, qui attribue une valeur nulle au travail des femmes. Un mystère, que seuls quelques économistes (en "mâle" de formules économétriques abstraites) arrivent à expliquer...

Une autre étude en effet, réalisée en Finlande en 1994, estime que la valeur monétaire du travail domestique représente au moins $42 \%$ du PNB. Si l'on calculait la valeur de ce travail au prix du marché, le taux pourrait même être supérieur. Cette étude indique également qu'une famille fournit en moyenne 7,2 heures de travail non rémunéré par jour, l'équivalent de 50,4 heures par semaine. Sur ce total, la part des femmes est de 5 heures par jour et celle des filles de 1 à 2 heures. Celle des garçons est inférieure à une heure par jour ${ }^{4}$.

L'attribution d'une valeur monétaire au travail domestique est une idée encore très discutée, mais qui fait cependant son chemin. La modification apportée par la 10e révision de l'AVS en Suisse, en 1994, reconnait par exemple au "salaire ménager" une valeur - fictive - de 33'000 francs suisses par an pour le travail domestique accompli par les femmes mariées.

\section{DIVISION SEXUELLE DU TRAVAIL}

Il existe une autre rupture paradigmatique entre travail et emploi: elle découle de la division sexuelle du travail. Notre vision du monde du travail est encore ancrée dans une conception patriarcale de l'organisation de la société, qui réserve aux hommes un accès prioritaire aux emplois salariés, alors que l'éducation des enfants et l'organisation du ménage incombe plutôt aux femmes.

Suite aux revendications des mouvements féministes, la législation concernant la division sexuelle du travail a été modifiée dans plusieurs pays occidentaux. Malgré ces changements, la répartition inégalitaire du travail et de l'emploi reste toujours profondément enracinée dans les mentalités et dans la réalité quotidienne de la condition féminine.

Le tableau suivant résume les multiples champs de tension qui traversent la problématique: emploi/travail, marchand/non marchand, formel/informel, salarié/non rémunéré.

\begin{tabular}{l|c|c|}
\multicolumn{1}{c}{} & \multicolumn{1}{c}{ Marchand } & Non marchand \\
\cline { 2 - 3 } Masculin & Emploi & Informel \\
\cline { 2 - 3 } Féminin & Temps partiel & Travail \\
\cline { 2 - 3 } & & \\
\hline
\end{tabular}

4. Cf. UMA DEVI S., Women, Work, Development and Ecology, New Delhi, Har-anand Publications, 1994, pp. 17-18. 


\section{DISCRIMINATION PROFESSIONNELLE, CHÔMAGE, EXCLUSION DES FEMMES}

Le troisième ordre de rupture entre le travail masculin et féminin est lié à la "configuration" interne du marché de l'emploi.

Le marché de l'emploi et l'organisation du travail dans les entreprises continuent à fonctionner selon des valeurs et des règles de comportements masculins. "Pensé par des hommes et pour des hommes", le monde du travail oblige les femmes qui s'y s'insèrent à se conformer à des principes masculins, au détriment d'un mode d'organisation qui tiendrait compte des valeurs féminines. De nombreuses enquêtes, effectuées aux Etats-Unis, mettent par exemple l'accent sur les difficultés rencontrées par les femmes diplômées en matière de reconnaissance de leurs compétences dans un univers masculin ${ }^{5}$.

Malgré l'augmentation de la main-d'oeuvre féminine dans les secteurs administratifs (aux EtatsUnis, 78,7\% des travaux administratifs sont accomplis par des femmes), de la santé et de l'éducation, les conditions de travail des femmes ne s'améliorent pas. L'inégalité de traitement entre hommes et femmes en matière de formation et de perfectionnement professionnel, ainsi que le poids des obligations familiales, constituent toujours des obstacles à l'émancipation professionnelle des femmes. Les tâches qui leur sont confiées restent le plus souvent routinières, subalternes et exigeant peu de qualifications professionnelles ${ }^{6}$. Ainsi, l'industrie micro-électronique dans les pays du Sud-Est asiatique fait massivement appel au travail féminin pour les postes peu qualifiés et mal rétribués (assemblage des modules, soudure des circuits électroniques ou contrôle de qualité).

Comment s'étonner dès lors que les emplois féminins soient les premiers marqués par la crise et les plus vulnérables lorsque se produit un changement de conjoncture? ${ }^{7}$ Selon l'OFIAMT, en Suisse et jusqu'en 1993, les hommes et les femmes ont été touchés par le chômage dans des proportions similaires (environ 4,5\%). Depuis 1994 en revanche, le taux de chômage des femmes est passé à 5,2\%, alors que celui des hommes est resté stable. En 1993, les femmes représentaient $41 \%$ des chômeurs, et cette proportion s'est élevée à 43\% en 1994. Les femmes sont également plus durement touchées que les hommes par le chômage de longue durée ${ }^{8}$.

La marginalisation sociale et son corollaire la pauvreté sont des phénomènes sociaux complexes qui accompagnent inévitablement le chômage. Leur évaluation - qualitative comme quantitative - varie en fonction du groupe social qui le vit. Il n'en reste pas moins que, derrière la relativité du concept de pauvreté, se cache la condition objectivement "sub-humaine" de la personne exclue?.

5. CORDERO Christiane, Le travail des femmes, Paris, Le Monde-Editions, 1994, p. 52.

6. HIRSCHHORN Larry \& WESTIN Alan, The employment effects of micro-electronics in the office and service sec tor in the United States, Geneva, ILO, 1986 (World Employment Programme Research, Working Papers). Par ailleurs, en Suisse, la Commission fédérale pour les questions féminines a mené une étude dans trois grandes entreprises pour analyser les effets de l'innovation technologique sur les postes de travail des femmes.

7. GREVE Rose Marie, The effects of technological change on women workers, International Industrial Relations Association, Seventh World Congress (Hamburg, 1-4 September 1986), 17 p.

8. Cf. "Les femmes lourdement pénalisées", La lutte syndicale (Revue des syndicats suisses), janvier 1995. 9. L'expression est de Fabrizio Sabelli qui parle de "la relativité d'un concept et de l'objectivité d'une condition". 
Avec l'exclusion correspondant souvent au cumul de facteurs socio-économiques et psychologiques (faiblesse de revenu, chômage, cadre familial instable ou éclaté, manque de qualifications professionnelles, difficultés relationnelles et/ou malchance), on peut aussi observer une tendance à la "féminisation" de la pauvreté. Les femmes seules ou cheffes de familles monoparentales constituent un groupe dont le risque de franchir le seuil de la marginalisation est élevé.

Avant d'aborder le partage du travail pour les femmes, il serait donc souhaitable que les partenaires sociaux et les pouvoirs publics se préoccupent d'abord sérieusement du travail des femmes et puissent répondre aux revendications suivantes: égalité de salaire entre les femmes et les hommes et accès des femmes aux possibilités de promotion, de formation et de recyclage.

\section{PARTAGE DE L'EMPLOI OU PARTAGE DU TRAVAIL?}

La montée endémique du chômage fait apparaitre l'idée du partage de l'emploi et du travail. Mais quel est le véritable objet de ce partage? A l'heure actuelle, l'objectif est de mieux répartir le nombre d'emplois entre le plus grand nombre de personnes actives. Une meilleure répartition des emplois et, partant, la réduction du chômage, pourraient être réalisées soit par la réduction du temps de travail, soit par l'introduction de l'emploi à temps partiel. Mais cette dernière forme ne concerne que très indirectement l'emploi féminin, le travail à temps partiel étant déjà largement adopté par les femmes.

Le temps partiel, "une forme d'emploi spécifique, au statut particulier, un mode d'emploi en tant que tel", pose en réalité de nombreux problèmes ${ }^{10}$. Il faut savoir en effet que dans les pratiques actuelles des entreprises - publiques ou privées - le statut des travailleurs (travailleuses) à temps partiel reste déprécié. L'introduction du partage de l'emploi à grande échelle, qu'accompagnerait sans aucun doute une féminisation accrue du temps partiel, comporte le risque d'augmenter la discrimination à l'égard des femmes, et de confirmer la dévalorisation de leur travail en termes de qualification, de rémunération, de profil de carrière et de stabilité de l'emploi ${ }^{11}$.

Par ailleurs, les femmes, du fait de la nature multiple de leurs tâches, choisissent généralement une méthode "frontale" pour les accomplir. "Les femmes ont une vision globale du monde, elles pratiquent une action non sectorielle et se sentent plus concernées par le vécu quotidien"12. Quand elles doivent assumer "de front" plusieurs activités comme le travail ménager, l'éducation des enfants, l'emploi professionnel et parfois certaines activités communautaires, elles se partagent. $\mathrm{Ne}$ pouvant compter sur le partage du travail domestique entre hommes et femmes, elles effectuent ainsi souvent de doubles ou triples (quand une responsabilité socio-politique s'y ajoute) journées de travail.

En conclusion, il y a donc bien une distinction à faire entre "partage de l'emploi", réservé aux salariés (au masculin) et "partage du travail" (au féminin). Pour développer le concept de partage de

10. MESSANT-LAURENT Françoise, "Travailler au féminin? Certes, mais à quel prix.," Campus (Magazine de l'Université de Genève), nº 27, mars/avril 1995, p. 21.

11. GAUVIN Anne, "Le travail professionnel des femmes. Vers d'autres modèles d'emploi?", Projet, n 236, hiver 1993-1994, p. 63; et BECHEREL Béatrice, "Temps partiel, mode d'emploi", Projet, n 236, hiver 1993-1994, pp. 67-74.

12. "Femmes et pouvoir chez les Verts", Le Bulletin Vert (Journal romand des Verts), no 2/95, p. 5. 
l'emploi et du travail tel que nous l'entendons ici, c'est un changement de paradigme qu'il faut envisager. On ne peut pas se contenter d'une réforme (réformette?) de l'aménagement du temps de travail qui s'inscrirait dans la continuité et changerait à peine les conditions de l'emploi existantes.

Afin de mieux répartir le travail féminin/masculin, des formes de travail innovatives doivent être imaginées. Il faudra prendre conscience qu'un véritable partage de l'emploi et du travail représente une rupture socio-économique, et devrait être inscrit dans un pacte de solidarité entre femmes et hommes, les générations actuelles et les générations à venir. Il s'agit de prévoir, dans un contrat volontariste et audacieux, une répartition plus équitable du temps social et professionnel, du travail et de l'emploi, des charges et des revenus ${ }^{13}$.

13. EUZEBY Chantal, "Les femmes et la protection sociale en Europe", Informations et commentaires (Salaire, Protection sociale, Exclusion, Emploi), no 89, octobre-décembre 1994, p. 38. 


\section{LES APPROCHES EMPIRIQUES AU FIL DES CONTINENTS: AFRIQUE, AMERIQUE LATINE ET CENTRALE, INDE}

Tenter de cerner la manière dont les femmes gèrent leurs problèmes d'environnement et de vie quotidienne dans les grands centres urbains, mène nécessairement à une multitude de pratiques si diverses qu'il est simplement impos sible d'en dégager des grandes lignes franches. En effet, au nom des différences interculturelles, il serait irrespec tueux de rapprocher les difficultés des femmes dans la guerre, par exemple, lorsqu'elles expriment des réalités aussi différentes que celles du Nicaragua ou celles du Mozambique. Les processus d'éducation ou d'accompagnements éducatifs de l'Inde et de l'Afrique sont tout aussi divergents comme le sont les densités de population de Bombay et $d^{\prime}$ Alger, sans parler des difficiles rapports hommes-femmes qui sous-tendent pratiquement toutes les situations.

Même si les stades de développement et les problèmes ponctuels peuvent être extrêmes à l'intérieur d'un même conti nent, il nous a semblé plus réaliste de regrouper des sociocultures et leurs écosystèmes par unité continentale. On nous pardonnera ces découpages sommaires puisque la richesse des différentes expériences nationales permettra au lecteur de tirer lui-même les enseignements qui l'intéressent. 


\title{
LES FEMMES ALGERIENNES: SPECTATRICES OU ACTRICES DANS LA VILLE?
}

\author{
FADELA CHAIB
}

Aborder le thème de la participation des femmes algériennes à la gestion de la ville et de l'environnement dans le contexte actuel n'est pas chose aisée. En effet, la crise multiforme qui secoue l'Algérie actuellement interpelle les Algériens sur les multiples raisons qui ont conduit leur pays à cette impasse. La situation des femmes depuis l'indépendance en 1962, est sans nul doute un de ses éléments clés.

\section{ESPACE PUBLIC ESPACE PRIVÉ: UNE COHABITATION CONFLICTUELLE}

Depuis les émeutes d'octobre 1988, date fondatrice de l'ouverture de l'Algérie au pluralisme politique, les femmes ont investi la scène politique en dénonçant leur mise à l'écart politique et sociale durant trois décennies sous le régime du parti unique, le FLN. Les plus militantes d'entre elles redoutent également de voir la mouvance islamiste gagner du terrain et imposer un projet de société qui repose essentiellement sur la négation de leurs droits.

L'Algérie s'est rapidement engagée sur la voie de la modernisation, pressée de rejoindre les autres nations après un siècle et trente ans de colonisation. La transition d'un modèle de société traditionnel à un autre moderne ne s'est pas fait sans tensions. En effet, la division spatiale des territoires entre les espaces privé et domestique traditionnellement dévolus aux femmes et l'espace public réservé aux hommes ne tient plus à cause notamment de la forte scolarisation des filles et leur accès au marché du travail. La rapide urbanisation du pays a élargi les opportunités du passage de l'élément féminin dans la ville. Cela ne s'est pas fait sans heurts. La violence contre les femmes prend de multiples visages: violence verbale et physique, injures, obstacles institutionnels et administratifs. Leur présence dans l'espace public a engendré des résistances au sein de la société.

Ces changements intervenus en l'espace d'une génération ont été d'autant plus rapides qu'ils ont provoqué des résistances. La rupture de l'équilibre de cette organisation spatiale entre l'espace privé et l'espace public a mis à nu des tensions sociales qui se sont manifestées en particulier par une violence à la fois diffuse et explicite. La rue est devenue pour les femmes un lieu de transit qu'il faut traverser à la hâte sans s'y attarder. Elles sont souvent l'objet d'injures, de harcèlements et même de violence physique. Il est courant que les auteurs de ces actes justifient de tels comportements en disant qu'une fille de bonne famille n'a rien à faire dehors, celles qui s y hasardent en assumant donc les conséquences. 
Le contrôle social et le cantonnement des femmes dans la sphère domestique et privée traduisent la peur de la fitna, ce fameux désordre sexuel et cette perturbation de l'ordre social que véhicule la présence des femmes dans les territoires masculins. Notre propos consiste à débattre des conséquences de cette transition vers la modernisation sur les représentations et les pratiques des femmes et des hommes quant à la gestion de la ville et de l'environnement. Indiscutablement le fait urbain a bouleversé le mode d'organisation de l'espace en faveur des femmes. L'espace domestique n'est plus l'unique univers pour des millions de femmes. Mais nous verrons que la rue - l'extérieur - reste toujours un espace "utilitaire" qu'il faut traverser sans s'y attarder.

En fait, l'Algérie fonctionne selon un double schéma: d'un côté elle aspire à la modernité sur le plan économique par le biais du développement et le passage vers une société de consommation, de l'autre la prégnance d'un système de type patriarcal et religieux, en définitive les résistances mentales traditionnelles à l'autonomisation des femmes, fragilisent la société et paralysent les efforts faits en amont pour arrimer le pays à la modernité. Le code de la famille est une illustration éclatante de cette schizophrénie.

\section{LE CODE DE LA FAMILLE: L'INSTITUTION DE L'INFÉRIORITÉ DES FEMMES}

Voté en 1984 par une assemblée conservatrice malgré la lutte difficile - en ces temps de pensée unique, d'absence de démocratie et de liberté de la presse - de quelques collectifs de femmes montre la victoire du courant traditionaliste. Ce code, anticonstitutionnel, est largement inspiré de la charîa. Il institutionnalise le statut inférieur de la femme et formalise la domination masculine sur les femmes dans pratiquement tous les domaines.

Une femme, qu'elle ait 18 ans ou 40 ans, ne peut se marier sans l'accord d'un tuteur, père, frère, oncle ou à défaut un juge. La polygamie, la répudiation, le divorce et l'héritage sont en faveur des hommes. La femme a seulement des devoirs, comme celui d'avoir des égards pour son mari en tant que chef de famille et pour les parents de celui-ci, la réciproque n'étant pas exigée. Le choix d'un travail salarié est soumis à l'autorisation du mari. La bataille autour de l'abrogation du code de la famille est le souci majeur des femmes aujourd'hui. Elles demandent le retour à des droits civils. Ce code fait des femmes des citoyennes de seconde zone. Comment peuvent-elles légitimement revendiquer le droit de participer à la gestion de la cité alors qu'elles n'ont que deux droits aux termes de cette loi: celui de gérer leurs biens et de rendre visite à leurs parents?

Les années 60 et 70 qui ont suivi l'euphorie de l'indépendance ont permis l'émergence des femmes sur la scène sociale et professionnelle. La situation a commencé à se dégrader au début des années 80 à cause du retour en force du discours religieux et des concessions des autorités de l'époque en manque de légitimité. Le cadeau royal offert aux islamistes a été le code de la famille. La crise économique qui a suivi la chute de la rente pétrolière et son corollaire le chômage ont constitué un cheval de bataille pour le Front islamique du salut (FIS). Ce dernier a en effet préconisé de renvoyer les femmes à leurs fourneaux et de libérer ainsi des postes de travail.

Malgré la scolarisation massive des filles, les femmes ne représentent que $8 \%$ de la population active du pays et 30\% d'entre elles sont de niveau universitaire. Les secteurs de l'éducation et de la santé seraient pourtant complètement paralysés si elles ne travaillaient plus car elles représentent plus de $80 \%$ des effectifs de ces secteurs. 
Le discours des islamistes a trouvé un écho favorable auprès de la société conservatrice qui reste sensible au propos religieux et démagogique. Certes, la présence féminine a opéré des fissures dans l'espace public; mais la femme a-t-elle pour autant acquis le droit de dire son mot et de participer à la gestion de son cadre de vie ou est-ce un domaine exclusivement masculin? Nous verrons que, contrairement à l'idée qui parait à première vue évidente, la question n'est pas tranchée. Cela tient aux pesanteurs des traditions mais aussi aux politiques de l'après-indépendance.

\section{LE VOILE ISLAMIQUE: UN AUTRE RAPPORT À L'URBANITÉ?}

Les femmes qui optent pour le voile ou le hidjab sont, à notre avis, au-delà de significations religieuses, révélatrices d'un autre rapport à l'extérieur. Elles clament leur droit d'accéder à l'espace public. En endossant cet habit, elles endossent aussi la foi et la vertu. Il leur sert de mur de protection. Elles érigent ainsi une barrière entre elles et les hommes. Elles prolongent l'espace domestique et veulent forcer le respect et renforcer l'acceptation de leur présence à l'extérieur des murs de la maison. Ce serait un sacrilège de manquer de respect à une "soeur musulmane". Certes, la première fonction de la femme dans l'esprit et la lettre de l'islamisme est d'être une épouse et une mère - cette exigence est aussi celle de la société traditionnelle - mais elle a également le droit et le devoir de s'instruire afin de transmettre le message religieux. Elle se doit donc pour ce faire de mener des activités sociales, politiques et professionnelles, ce qui n'est pas prévu dans le contrat sociétal traditionnel. Elle a accès à la modernité par le biais de la religion dans sa version engagée. Pour paraphraser L. Bucaille: en se voilant, elle a payé une sorte de droit d'entrée à la vie extérieure et débouche sur une émancipation relative'.

Le voile permet un autre rapport à la société patriarcale. En fait, ces femmes clament "que leur soumission à Dieu est plus forte que la soumission au père". C'est aussi une forme de contestation, la remise en cause de la toute-puissance parentale et la production de nouvelles valeurs. Grâce au voile, elles renversent les rapports de force et de domination. Ainsi habillées, possédant la connaissance coranique, soumises à Dieu et moralement irréprochables, elles deviennent des interlocutrices incontournables et écoutées. La recherche de l'autonomie est concomitante avec cette affirmation identitaire. La femme ou la fille n'est plus cette personne éternellement soumise au contrôle de la famille. L'islamisation bouscule les réseaux de solidarité traditionnelle au profit de structures de nouvelles communautés: celles des frères et des soeurs.

En fait elles deviennent quelque part responsables d'elles-mêmes et de l'honneur de la famille. Elles réactivent cette obéissance aux lois et valeurs sociales en lui donnant un contenu islamique et individualisé. Les parents et les frères relâchent sensiblement la surveillance qu'ils exercent sur elles. La hiérarchie s'inverse dans la cellule familiale Ce sera la fille - être minorisé et dominé - qui va édicter un certain nombre de règles de conduite. Dans ce cas, le voile sert à renverser la hiérarchie des âges et à donner une légitimité morale aux filles par rapport aux mâles de la maison.

Les femmes n'adoptent pas toutes les mêmes stratégies de contournement Elles rusent avec la société pour acquérir le droit à la ville et à l'extérieur. Celles qui se voilent acquièrent ainsi un

1. BUCAILLE L., "L’engagement islamiste des femmes en Algérie", Magbreb-Machrek, nº 144, 1994, p. 108. 
droit de passage dans le territoire hostile des hommes. Celles qui refusent le port du voile clament au contraire qu'elles n'ont pas besoin de s'entourer des pans de leur voile pour se faire accepter. C'est à la société de s'adapter à leur présence et de les accepter comme êtres à part entière. La féminité n'est pas une tare qu'il faudrait voiler pour la neutraliser et éviter la fitna, estiment-elles.

\section{LA BATAILLE AUTOUR DE LA MIXITÉ}

L'exigence de la non-mixité est très présente dans la société algérienne. Les écolières et les écoliers sont séparés sur les bancs de l'école et dans les cantines. Les administrations, les salles d'attente des hôpitaux ou des cabinets médicaux ne sont pas communes. Aux bureaux de poste ou aux guichets des services municipaux, il y a des files pour chacun des sexes. Les transports en commun restent le lieu de côtoiement par excellence. Les femmes obligées d'emprunter les bus deviennent les victimes expiatoires des frustrations sexuelles de leurs compagnons de voyage. Pincements et attouchements, favorisés par la proximité, sont le lot quotidien de milliers de femmes. Celles qui osent se rebiffer sont traitées de tous les noms avec l'assentiment quasi général de toute l'assistance masculine. Les hommes et les femmes se rencontrent et se côtoient dans des rapports de violence et de domination.

\section{LA NAISSANCE DU PHÉNOMÈNE ASSOCIATIF}

Si, à l'évidence, les hommes sont souverains dans la ville, ils ont été tenus à l'écart par l'Etat et ne participent pas à la gestion de leur ville. Avant 1988, aucune association politique ou sociale qui n'émanait pas des instances du FLN n'était autorisée à être active. Les autorités algériennes se sont toujours méfiées du fait associatif. La société était encadrée et contrôlée. La brèche est survenue avec les événements d'octobre 1988. On assiste alors à un extraordinaire foisonnement d'associations, de comités et d'organisations. Une véritable remise en cause des rapports entre l'Etat et la société s'instaure et englobe un débat plus large: celui des libertés publiques et de la démocratie.

En quelques mois, plusieurs centaines d'associations ont vu le jour. Cette explosion du phénomène associatif a abouti à la prise en charge par la société de plusieurs créneaux qui étaient soit maintenus tabous par les autorités soit mal représentés: les mères célibataires, la condition féminine, les droits de l'homme, l'enfance abandonnée, l'éducation et les questions d'environnement. Ce phénomène est l'illustration de la faillite du système d'assistanat et de l'Etat-providence.

Toutefois, la palme revient aux comités de quartiers qui ont fleuri en particulier dans les cités et les ensembles d'immeubles, le but étant l'amélioration du cadre de vie, le règlement de certains problèmes, en fait remédier à la démission des services publics et de la voirie. Ce sont souvent les femmes qui poussent les hommes de la famille à s'investir dans de telles actions. Ce sont elles qui s'inquiètent pour la santé et la sécurité des enfants. Il n'est pas rare de les voir leur donner des instructions du haut de leurs balcons.

Curieusement, les voisins ont commencé à se connaître et à se côtoyer à cause des antennes paraboliques pour capter les télévisions étrangères. En effet, la mobilisation des habitants pour l'achat et l'installation de ces antennes collectives, afin de rompre le conformisme de l'unique 
chaîne nationale, est à l'origine de la création de plusieurs syndicats d'immeubles. Cet achat important effectué, les voisins ont commencé à s'intéresser à d'autres problèmes tels que la création d'aires de jeux, les campagnes de nettoyage, et les cotisations pour payer des gardiens d'immeubles ou de parking. Les femmes ont commencé à se connaître et celles qui ne travaillent pas gardent les enfants de celles qui ont une activité au dehors. Cette aide est la bienvenue en l'absence de structures d'accompagnement comme les crèches et les jardins d'enfants.

La prise de conscience de la société quant à l'importance de son implication dans la gestion de la ville est récente. Avant cela, on s'en remettait aux autorités publiques pour tout. Aujourd'hui, l'argent manquant, ces dernières sont contentes de se décharger de plusieurs tâches. Il faut dire que le pays ne possède pas des traditions de travail associatif et communautaire.

La campagne fait exception parce que les intérêts y sont communs et les gens de la même origine tribale et géographique. L'entraide est indispensable pour effectuer certains travaux lourds comme les moissons, la construction de puits, de hangars ou de maisons.

Les femmes algériennes s'investissent plus dans la création d'associations féminines et de protection de l'enfance. Les femmes islamistes ont créé des associations caritatives et font un travail de proximité. Elles luttent contre l'analphabétisme et proposent des formations aux jeunes filles dans la couture ou d'autres travaux. Elles préfèrent l'engagement culturel et social à la lutte politique.

\section{LE MOUVEMENT FÉMININ ET LA LUTTE POUR LES DROITS POLITIQUES}

La première manifestation des femmes le 8 mars 1989 après l'ouverture du pays au pluralisme politique a agi comme un électrochoc. Pour la première fois depuis l'indépendance, des milliers de femmes ont pris possession de la ville pour quelques heures. Elles ont exprimé leurs revendications comme la promotion de la femme, l'abrogation du code de la famille, la citoyenneté totale et la lutte contre les discriminations. Les hommes, étonnés de voir autant de femmes dans leur espace, ont découvert à cette occasion que la moitié de la population était mécontente de son sort.

Depuis lors, les femmes dans la rue sont une réalité permanente. Aujourd'hui elles sont à l'origine des plus grands rassemblements sur la place publique malgré le climat de terreur et de violence qui règne. Elles manifestent contre le terrorisme, la violence, et n'hésitent pas à descendre dans la rue chaque fois que la nécessité de combat se fait pressante. Les hommes ont changé d'attitude à leur égard et reconnaissent volontiers que, si le pays n'a pas encore totalement sombré, c'est grâce à la résistance quotidienne des Algériennes. Elles savent qu'elles ont tout à gagner et plus rien à perdre. "Ce sont elles les hommes de l'Algérie", "femmes, on vous aime" titrent certains journaux indépendants.

Les femmes algériennes ont conscience aujourd'hui que les droits politiques sont la pierre angulaire de toute émancipation. Elles savent que l'instauration d'un Etat islamique représente un danger évident pour l'acquisition du statut de citoyenne. Les islamistes n'ont jamais caché le sort et la place qu'ils leur réservent dans leur proiet de société. C'est la lutte principale des Algériennes à l'heure actuelle puisque la plupart combattent le discours islamiste et ne font plus confiance au pouvoir politique en place. 
Il est évident que le mouvement des femmes est devenu incontournable sur la scène politique. Les femmes, de la génération de l'après-indépendance, veulent être les dignes filles des figures emblématiques de l'Algérie, celle de la Kahina, de Fatma n’Soumer, de Hassiba Ben-Bouali. Elles luttent pour que l'avenir ne se dessine pas sans elles et surtout qu 'il ne se fasse pas à leur détriment. Les exemples historiques sur l'utilisation des femmes en temps de crise et la négation de leurs droits une fois la paix revenue ne manquent pas. L'Algérie, et par extension le monde arabo-musulman, doit régler le contentieux de la condition féminine qui étouffe la société entière. Les femmes ne se battent pas seulement pour elles, ni contre les hommes, mais pour une vie saine et policée. A l'heure où les murs et l'apartheid tombent, il devient indécent de nager à contre-courant de l'histoire et de la nature. 


\title{
Women in Conflict Situation :
}

\section{Gender IsSues in Transition FROM War to Democracy IN NAMIBIA AND MoZAMBIQUE}

\author{
MARNi PIgOTT
}

The title refers to three complex areas - conflict, gender and transition politics. Each of these areas could provide a focus for a lengthy paper. This brief exposé attempts to explore some of the interrelationships between the three areas in the light of the overall thematic concern "women in dramatic change".

In order to better understand these relationships, it is necessary to make some broad observations around conflict and gender. From the perspective of gender, conflicts can be identified at two different levels, on one hand at the individual or individual household level, in the so-called private sphere, and on the other hand at the level of the community, in the public sphere. Conflicts in the public sphere can involve neighbourhoods, clans, ethnic groups, communities. They can assume local, national or even international dimensions as the crisis in former Yugoslavia illustrates. It is almost a truism to say that the impact of conflict at community level can be very dramatic at the household and individual level. It is certainly true to state that there is a gendered experience of conflict at community level. "Ethnic cleansing" for instance involved the large scale and systematic use of rape as an explicit strategy in country contexts as different as former Yugoslavia and Rwanda.

Apart from these extreme cases of gendered conflict, other conflicts are resulting from the impact of global processes such as economic restructuring, political transformation, market integration, rising demographic imbalances between rich and poor countries, unemployment, diminishing resources and environmental degradation among others. Increasing disparities and social differentiation along the lines of race or gender are contributing to the enormous stresses being experienced by particular groups of women and men in many different contexts, either at the level of the individual of individual household, or at the community level, or both.

The reference to women and men is deliberate. To speak in terms of gender is not only useful - it is necessary. Gender as a social relation embraces both women and men. Women and men are involved in conflict situations, both as actors and as victims. And women and men are involved in transitional periods following conflicts. In the process of transition from war to democracy, for instance, women and men are actors - both contributing to social transformation. Conceptually speaking, gender as a social relation expresses the socially constructed roles of women and men in the private and public spheres. The particular gender roles of women and men are not restricted 
to production and reproduction of the household but also encompass decision-making and political activities in the wider community. The gender division of labour is in turn influenced or reinforced by the relations of access to and control over different resources, which women and men may have in a specific society of community. For instance, women farmers may have access to land for food production, but do they have control over any surplus or access to cash from the sale of surplus? A similar question can be posed concerning access to urban land. Is it only through relationships to men that women as wives of "dependents" can get titles to land? In this sense, structures of access of exclusion may be seen to operate through customary or legislative practice, creating and sustaining gender differentials, and not infrequently giving rise to "gendered" social conflicts.

It is therefore important to recognise four points concerning gender relations:

Gender intersects with other social relations or social identities such as class, ethnicity, religion, rural and urban, age, etc. It is not therefore a question of all women or all men, but multiple and sometimes contradictory identities. This means in conceptual and practical terms that women cannot be seen as a unitary category. Women are not a homogeneous group. Neither are men.

Gender relations are context specific . Even within the same country there may be significant differences in gender relations. In Mozambique, a matrilineal society in the north of the country contrasts with different gender relations in the south.

Gender relations can and do change. Under the impact of colonialism, for instance, gender roles have clearly changed in many countries in Africa. An example is the case of Mozambican men being recruited for work on the mines in South Africa and the impact of this exodus on rural production relations with the increasing role for women in agriculture. But in addition, change is a response - often a pragmatic response - to conditions of scarcity, of part of a survival strategy in situations of conflict. And these changes can and do take place both at the level of the household and the community.

Like other social relations, which find expression in socio-economic and political institutions as well as in societal processes, gender relations also resist change. This resistance is frequently expressed in attitudes, for example, towards women in politics and leadership positions; or in assumptions about "the family" and stereotype perceptions of men and women's work. Extreme resistance expresses itself as conflict of violence, either domestic or communal violence, and often directed against women.

Theses four related observations together provide a kind of lens or perspective through which issues in conflict situations and transition processes can be examined. The cases of Mozambique and Namibia can illustrate some of the implications for gender relations which are suggested in the process of democratisation.

Democratisation is clearly a global political theme, and throughout the world democratic forms of political constituents of societies are under discussion. Women and men are actors in the process, but it appears that their roles in shaping social transformation differ in some significant respects. In some cases, political observers refer to gendered perceptions of democracy and to the gendered impact of the outcome of democratic transition processes. Moreover, and this needs to 
be restated, the experiences and impacts of wars or conflicts which precede transition processes are themselves gendered in several ways.

In Mozambique, for example, there is no question about the devastating impact of the 15 year war during which more than one million people were killed and more than four million people were made refugees inside and outside the country. Women made up the bulk of the adult population of the refugee camps, often up to 70 per cent. Within Mozambique, women in communities of displaced population lost many of the social support mechanisms, which had previously provided a minimum of security. Widows and women abandoned by or separated from husbands were particularly disadvantaged in their ongoing struggle for their own and their children's survival. The experience of Mozambican women, often very young, who were seized vy RENAMO soldiers to provide domestic, agricultural and sexual services is another specifically gendered aspect of the war.

Tracing the process from this devastating war to the peace accords, which were signed in Rome in October 1992, and the process from a single party state to the holding of multi-party presidential and parliamentary elections in October 1994 is extremely complex. The accords committed FRELIMO and RENAMO ${ }^{1}$ to the demobilisation of their soldiers, to assisting the process of reintegration and resettlement of millions of people, the registration of over eight million of Mozambican women and men for elections, and the transformation of RENAMO from an armed organisation to a political party. It is important to note, however, as pointed out by Jacobson that the "principal dynamic behind the decision to hold multiparty elections in Mozambique was not, as with Malawi and Zambia, a sustained process involving a wide range of representation from civil society. Instead, Mozambique has been much more specifically affected by external global forces. ${ }^{2}$ "The emphasis on peace and reconciliation in turn imposed specific conditions on the transition process, as the peace process had become equated with the achievement of multiparty elections. According to Jacobson, "what might in other contests have been an authentic and healthy political conflict became a potential threat to the fragile equilibrium reached since the peace accord in $1992 \ldots$ Women, who had borne the brunt of the suffering, were particularly bound up in this, as some aspects of their experiences, specially that of sexual violence, are particularly difficult to voice." "But what of some of the other gender issues evident in Mozambique's transition process? And what are some of the implications?

Firstly, it is important to recognise the nature of gender constraints on the ability of women to participate at the formal political level. The divisions between the public and private spheres are deeply entrenched in Mozambique, with women clearly restricted in large part to the private/domestic domain, apart from their important public role in some community rituals. But at the same time, women have substantial experience in community organisation at the more informal political level. Thus, taking the overall electoral process as an example, while women were largely exclude from the public sphere by i.a. their inability to speak Portuguese which is

\footnotetext{
${ }^{1}$ FRELIMO, the Front for the Liberation of Mozambique, conducted the armed struggle for independence from 1964. With independence achieved FRELIMO converted itself into a Marxist Leninist party. RENAMO, Resistencia Nacional Moçambicana, was created by the then Rhodesian Central Intelligence Office following the Mozambique government's compliance with United Nations sanctions against Rhodesia. With Zimbabwean independence in 1980, South African Military Intelligence took over the training, arming and management of this force.

${ }^{2}$ Jacobson R., "Dancing towards a better future? Gender and the 1994 Mozambican Elections". Report prepared for NORAD, November 1994, p. 10.
}

${ }^{3}$ Ibid., p. 11. 
Mozambique's official language ${ }^{4}$, women's organisations of various types were crucial for the success of the civic education programmes. This is because their existing community based structures provided access to the non-literate sectors of the urban and rural population. Nonetheless, according to some observers, women's organisations found themselves marginalized in terms of resources such as transport around the rural districts, and as a result would have been less visible in an important public sphere.

Secondly, it is important to examine the gender profile of political parties and the position of women and men candidates on party lists. Here FRELIMO adopted an explicit policy decision on affirmative action - one third of places on party lists were allocated to women and another third to "young people". This quota system for candidates was similar to the one used by the ANC in the South African elections earlier in April 1994. According to FRELIMO's statements, the affirmative action strategy resulted from their recognition of the demographic structure of the population plus the vital role which women have always played in the party, in government and the nation. In fact, 37 per cent of the candidates presented by FRELIMO were women whereas RENAMO had only 9 per cent women candidates. The final results, announced on 19 November 1994, revealed that women had obtained 24.4 per cent of the seats in the Assembly with 61 out of a total of 250 deputies.

There is an important gender issue here and an ongoing debate concerning representatives whether women or men - and representation of gender interests. Clearly what is specially relevant is how representatives contribute to the process of defining gender responsive policy and appropriate programmes which an redress existing inequities and gender differentials as part of the overall process of reconstruction. In the case of Mozambique, one critical issue will be the question of land, and how existing constraints on access for women in many contexts can be overcome. This crucial debate, however, was completely absent from the political platforms of either of the major political parties in the electoral campaign. There is another related issue concerning the renewed interest in reaffirming the right of traditional leaders to represent their communities. This aspect has become linked with the role of decentralisation and the democratisation debate in several African countries. In Mozambique's case, it also stems from the need to consolidate the process of reconciliation and also FRELIMO's desire to win over segments of Mozambican society, which had been alienated as a result of the earlier policies against traditional authorities. However, there are gender implications, as traditional authority structures are often based on notions which preclude equality of participation by men and women, despite the fact that some women are present in theses structures.

In the case of Namibia, the process of transition involved a lengthy struggle for independence, in which women were active as PLAN fighters, and a protracted involvement by the international community. The process culminated in the first democratic elections for a Constituent Assembly held in November 1989. There has therefore been a five year period for the consolidation of institutional democracy. Clearly, there are many differences in the experience of the two transition processes, not least because of the size of the populations involved whereby many more women of

\footnotetext{
${ }^{4}$ In 1991, women represented $75 \%$ of the rural population aged 20-24 who could not speak Portuguese and $80 \%$ of the urban population. Swedish International Development Authority (SIDA), MozambiqueCountry Gender Analysis, 1993.

${ }^{5}$ This information comes from the analysis of local government elections undertaken by Hubbard D. and Kaveri K., "Affirmative Action for Women in Local Government in Namibia", Legal Assistance Centre, Namibia, June 1993.
} 
Mozambique's 16.5 million inhabitants had been affected by the war than in Namibia with an estimated overall population of 1.7 million people.

In the first election for Namibia's Constituent Assembly in 1989, 5 out of 72 elected members were women, which represents 7 per cent. This percentage at the national level compares with 24.4 per cent in the Mozambican parliament. The local government elections held in Namibia in November 1992, however, provided very interesting results concerning women's participation at the local level ${ }^{5}$. There were two types of elections, local authority elections for municipal councils, town councils and village councils on one hand, and regional council elections on the other. In the case of the former, it is important to note that the Local Authority Act of 1992 made provision for affirmative action. Each party list had to contain two or three women as candidates. Elections for regional councils made no such provisions, and women comprised only 12 per cent out of 195 candidates. Three were elected, giving a 3.16 percentage representation to women on regional councils. By contrast, women constituted 38 per cent of the total candidates for local authority elections, and the results gave 26.4 per cent in town councils and almost 34 per cent of seats in village councils to women. Four village councils have women in the majority.

The affirmative action provision may indeed have contributed to the higher participation of women in local elections as compared with regional and national elections. But there is another important aspect to the Namibian example which relates to an earlier point, namely the possibility of gendered perceptions of institutional democracy. In this regard, despite the narrowness of the concept "institutional democracy", it is possible to distinguish between the types of issues dealt with by institutional structures at different levels. Thus, there is the perception that critical areas of community interest such as water, education, health sectors - which are of direct and specific concern to women because of their gender roles - can and should be lobbied for at the local level. In the case of Namibia, these are the village, town and municipal councils, which reflect an active participation by women in the electoral process.

This participation, however, is also the formal political expression of a long history of Namibian women organising in various community based organisations at the community level. Women have experience, and it is now becoming visible in the formal political arena particularly at the local level. Mozambique is scheduled to hold elections for local government in the future, and it will be very important to monitor the gender dimensions of that process.

Il seems probable, due to various types of gender constraints, that it is easier in some respects for women to participate politically at the local level. (This is borne out by recent experiences in many European countries.) But clearly, major resource allocations and the establishment of policy and legislative frameworks are being debated at a higher level and frequently without informed discussion on the potential gender implications of such decisions these implications having their impact at the local level. Thus there is a need for strategies to be designed in an integrated way, to take account of the interrelationships between institutional structures at different levels and with various actors and groups in civic society.

The process of consolidation of democratisation, leading to more equitable systems of access to resources and opportunities, clearly requires going beyond narrow definitions of institutional democracy. Only a gendered approach to these questions is likely to ensure an effective transition to effective democracy. 


\title{
REPARTITION DES ROLES ENTRE HOMMES ET FEMMES EN ZONE PERIURBAINE AU MOZAMBIQUE
}

\author{
MARGARITA MEJIA
}

$\mathrm{Au}$ sein de notre institution ${ }^{1}$, les enjeux concernant les questions liant genre et environnement sont traités au sein d'un programme intégré sur le développement durable, commencé en 1992. Dans ce cadre, des consultations ont été réalisées, en particulier une étude d'impact social et environnemental dans le cadre du Plan national de Reconstruction. Nous avons également réalisé des évaluations portant sur les infrastructures, les services urbains et la dimension de genre, dans des zones périurbaines.

Notre Département a aussi été impliqué dans des missions de formation sur les méthodes de planification de genre, d'abord à Maputo puis dans les Provinces du nord et du centre. Ces cessions ont mêlé apprentissage théorique et travaux de terrain, pour élaborer des diagnostics sur la situation des femmes, en particulier en ce qui concerne l'accès aux ressources.

Notre dernière recherche en date porte sur "Prognostic on environmental managment in intermediate cities, public policies and local dynamics". Ce projet fait partie d'une recherche plus large sur "Villes en développement" de l'Institut universitaire d'études du développement à Genève, financée par le Fonds national suisse de la recherche scientifique (FNRS). Un certain nombre de résultats montrent, en zone périurbaine, la modification des rôles des hommes et des femmes et les liens entre zones rurales et urbaines, maintenus surtout à travers l'agriculture de subsistance des femmes. Il ne s'agit pas ici d'en faire une description détaillée ou chiffrée.

\section{FEMMES ET ENVIRONNEMENT AU MOZAMBIQUE}

Nous aimerions décrire les efforts de femmes et d'hommes pour survivre et assurer un développement minimum en zone périurbaine, dans l'un des pays les plus pauvres du monde. Le Mozambique est en effet un pays en transition, passé du statut de colonie à celui de pays indépendant, d'un système socialiste et centralisé à une économie de marché, au multipartisme et à la décentralisation, de la guerre à la paix. Nous voudrions analyser comment les rôles et les relations entre femmes et hommes ont évolué dans ce processus, et en particulier ce qui s'est passé au sein de la population la plus vulnérable, dans ces espaces particulièrement fragiles que sont les zones périurbaines.

1. Le Centre d'Etudes Africaines de l'Université E. Mondlane poursuit des activités de recherche depuis 1977. Le département d'études sur les femmes et les questions de genre a été créé en 1987. Depuis 1992, les questions de genre et d'environnement et leurs enjeux sont intégrés au sein d'un programme de recherche sur l'environnement durable. 
Pendant la colonisation portugaise qui s'est terminée en 1975, les caractéristiques des relations de genre se présentaient de façon différente dans des zones géographiques où ressources humaines et naturelles étaient exploitées de façon variable:

- dans le nord, les femmes coloniales recrutaient la force de travail masculine, et parfois les femmes devaient produire les cultures intensives (coton, noix de cajou, thé pour l'exportation) à l'intérieur du champ familial;

- dans le centre du pays, le recrutement de la force de travail masculine était effectué par les multinationales agroindustrielles, par exemple dans les exploitations de canne à sucre, ce qui obligeait les familles à déménager dans des camps isolés. A la même époque fut créée la "Compagnie du Mozambique" qui devint une très grande entreprise. Son activité principale était le transport, de l'intérieur vers la province de Beira, et elle avait son propre territoire, son économie, ses lois, en concurrence avec le pouvoir portugais;

- au sud, la force de travail masculine était vendue par le gouvernement portugais, presque à la façon des esclaves; les hommes allaient ainsi travailler dans les mines d'or sud-africaines.

Tous ces faits conduisirent à une évolution des relations de genre par rapport à la société africaine. Dans presque tous les cas, les femmes durent étendre leurs responsabilités à l'ensemble des tâches concernant la famille, tandis que le rôle des hommes était cantonné à la production.

L'indépendance, dans un contexte d'influence soviétique, apporta l'émancipation des femmes. A vrai dire, les femmes avaient largement participé au combat pour l'indépendance non seulement sur le plan logistique et de la nourriture mais aussi en combattant aux côtés des hommes.

Le mouvement de libération mozambicain (FRELIMO), créa l'Organisation des Femmes Mozambicaines (OMM) qui assura la promotion de la participation des femmes au niveau politique et communautaire. Les objectifs de l'OMM étaient de permettre aux femmes de s'émanciper de pratiques traditionnelles telles que les rituels d'initiation et la dot (lobolo), et d'obtenir un accès égalitaire à l'éducation et la reconnaissance constitutionnelle de droits égaux pour les hommes et les femmes.

La guerre civile, qui a commencé en 1980, était à l'origine une lutte pour le pouvoir et devint, à cause des intérêts extérieurs, une guerre de déstabilisation. Son objectif principal était la destruction des infrastructures économiques et sociales en zone rurale. Cela entraîna un exode dans des zones plus sûres autour des villes: les zones périurbaines s'étendirent donc de façon incontrôlée, occupant en général des terrains impropres à la construction. Il en résulta une profonde cassure des structures familiales et du tissu social. L'état d'urgence fut proclamé et de nombreux camps de réfugiés furent créés. Hommes et femmes devinrent dépendants de l'aide humanitaire pour leur survie.

Finalement le FRELIMO, désirant la paix, a renoncé à sa philosophie et à sa ligne politique en acceptant les principes du multipartisme, le programme d'ajustement structurel, l'économie de marché et la décentralisation. Le rôle social de l'Etat a ainsi perdu de son importance et la priorité est maintenant à l'efficacité dans le remboursement de la dette. 
L'éducation, la santé et les services urbains doivent être privatisés. Au niveau de la communauté en périphérie urbaine, ils sont encore sous la responsabilité de la structure communautaire qui a été créée dans un contexte socialiste par le FRELIMO, lequel a désormais perdu son statut avec l'introduction du multipartisme. Du fait que les services sociaux sont considérés comme non productifs (parts d'une fonction "reproductive"), ce sont les femmes qui en assument la responsabilité.

Le Plan d'ajustement structurel, dans son effort pour créer un système économique efficace, a une ligne de conduite visant à utiliser aussi peu de force de travail que possible, sans alternative face à une situation de chômage qui affecte principalement les hommes et qui est encore amplifiée par le processus de démobilisation. Les femmes sont tout aussi affectées mais moins directement puisqu'elles doivent assumer leur rôle productif à travers le marché informel.

Cette situation tend à se généraliser au Mozambique avec les caractéristiques suivantes: la relation de parenté, le système matrilinéaire ou patrilinéaire et cette tendance nouvelle, "post-moderniste", à vouloir retourner au système traditionnel. Ce dernier comprenait une bonne dose de discriminations de genre, qu'il s'agisse de la participation des femmes dans le processus de décision ou de l'utilisation et de l'accès aux ressources (travail productif, crédit, éducation et technologie).

La question de l'égalité des hommes et des femmes est abordée dans la Constitution mozambicaine mais, dans la pratique, au moins $80 \%$ de la population mozambicaine ignore ce fait et agit selon la loi coutumière.

Les zones périurbaines présentent une situation bien particulière du fait des influences que le contexte politique volatile entraîne dans un espace sensible et vulnérable; il s'agit d'une population de migrants des origines régionales et des traditions diverses, avec des conditions environnementales spéciales, des rôles et des relations de genre différents qui créent leurs propres dynamiques de survie et mécanismes de sécurité.

Nous désirons présenter ici trois cas montrant trois zones périurbaines, au nord, au centre et au sud du pays.

\section{OBISPOIS}

Obispois est un district à la limite de la ville de Nacala, dans la province de Nampula au nord du Mozambique. Le système est matrilinéaire mais il faut souligner que matrilinéaire ne signifie pas matriarchie: les décisions sont toujours prises par les hommes. C'est plutôt une relation de genre différente avec moins de discriminations pour les femmes, en particulier en ce qui concerne l'accès à la terre, et une meilleure relation entre hommes et femmes, faits qui donnent l'impression, vu d'autres régions, que les femmes ne travaillent pas à la maison selon la coutume.

Nacala dispose des meilleures conditions naturelles pour un port, sur l'ensemble de la côte est de l'Océan indien. Par contre, la ville n'offre pas de bonnes conditions pour la construction de logements, les terrains étant très pentus tout autour des installations portuaires.

Les familles des travailleurs et tous ceux qui se trouvaient associés au trafic portuaire ont construit leurs logements dans des zones peu propres à la construction, une forte érosion ayant 
provoqué un désastre pendant l'hiver 1988. Ces événements, de même que le cyclone "Nadia" en 1994, ont obligé les femmes à s'impliquer dans les activités de construction qui relevaient, traditionnellement, des hommes. Une autre activité touchant à la gestion de l'environnement, assumée à la fois par les femmes et par les hommes, est la participation toujours bénévole au programme de contrôle de l'érosion des sols.

\section{BEIRA}

Au centre du pays, Beira est la capitale de la province de Sofala, dont certaines zones sont endessous du niveau de la mer. Elle a été créée à cause de l'importance stratégique du port et du corridor qui relie le Zimbabwe à l'Océan indien. Pour cette raison, pendant la guerre, ce corridor était protégé par une force spéciale de sécurité, ce qui constituait un élément très attractif pour des populations civiles fuyant des territoires ravagés par les combats.

Les moyens de transport, principalement le chemin de fer, rendaient relativement facile l'arrivée de ces populations déplacées à Beira, et plus spécifiquement, à sa périphérie. Il faut comprendre que ces familles déplacées n'étaient en général pas au complet. Les groupes arrivaient de la même région, mais les familles se trouvaient écartelées, certains de leurs membres étant captifs, perdus ou morts.

Cette situation sociale très complexe a été le lot commun des habitants de la périphérie de Beira; mais quelles sont les caractéristiques physiques de ces zones d'habitat? Mis à part l'absence d'infrastructures et l'occupation non contrôlée, les zones périphériques de Beira city ne sont pas propres à la construction de logements car l'eau affleure partout. La "ville en dur (ville de ciment)" a pu être construite, à l'époque coloniale, grâce à des travaux de remblaiement; mais les zones périurbaines sont trop basses pour qu'on puisse envisager de construire la moindre infrastructure, et même des latrines de fortune contamineraient l'ensemble de la nappe.

Le quartier de Munhava constitue un exemple typique dont il est important de considérer l'impact sur les relations hommes-femmes. Comme nous l'avons vu plus haut, la composition de la population rendait difficile l'adaptation à ce contexte nouveau et une telle adaptation n'apparaissait pas possible sans une modification des rôles traditionnels des hommes et des femmes.

A cause de la guerre, l'homme de la famille était le plus souvent absent et la femme devait assumer des rôles qui dans la tradition n'étaient pas les siens, tels que construire la nouvelle maison ou assumer les dépenses quotidiennes. Cela venait en plus des devoirs habituels: chercher l'eau, trouver et préparer la nourriture. Même ces tâches ont été rendues très difficiles à cause de la contamination de l'eau, de la difficulté de trouver un champ et de la nécessité de tout apprendre d'un environnement nouveau.

La seule façon de survivre était d'entrer dans l'économie informelle. Cette stratégie du minimum vital consiste à vendre un peu de bois ou de charbon ou des pierres pour la construction, revendre des produits agricoles achetés au marché de la ville, etc., presque toutes ces activités étant assurées par des femmes. Dans cette lutte pour la survie, les gens ont recherché des quartiers plus proches des marchés, tel que Gotto. Il s'agit d'un quartier périphérique impropre à la construction car situé sur des basses terres, mais proche du centre de la ville, de ses services et des opportunités d'emploi pour les hommes et des marchés informels pour les femmes. 
Dans la plupart des maisons que nous avons visitées dans le cadre de notre recherche, nous avons trouvé des hommes au chômage, prenant soin de la maison tandis que les femmes étaient parties chercher de l'eau ou s'occupaient au chingamolo (nom du marché informel en langage local). L'état d'esprit à Gotto était et reste très agressif dans un contexte de lutte pour la survie. Ce fait inquiète le voisinage huppé car, ironiquement, Gotto est situé à proximité immédiate de l'une des banlieues les plus chics de la ville. Il en est résulté de nombreuses pressions auprès de la Municipalité pour obtenir l'évacuation du quartier. Si nous analysons les différentes raisons données par les habitants de Gotto pour demeurer dans un quartier où il semble que tout se ligue contre eux, les hommes parlent de possibilités d'emplois et les femmes des services d'éducation et de santé qu'elles trouvent dans le voisinage.

De toute façon, les fortes tensions subies par les habitants les obligent à travailler ensemble pour surmonter leurs problèmes, en particulier par rapport à l'environnement. Il y a donc solidarité et partage des rôles pour trouver des solutions, par exemple combler les parties les plus basses du quartier en vahies par l'eau ou gérer les ordures.

\section{Mavalane et Hulene}

Cette zone suburbaine de Maputo est bien sûr très surpeuplée du fait qu'il s'agit de la capitale d'un système centralisé. Elle n'a pas les problèmes environnementaux habituels que rencontrent les autres régions, mais elle doit surmonter les conséquences de la surpopulation et de la croissance rapide, en l'absence d'infra-structures et de services urbains et sociaux.

Nous avons été en contact avec les quartiers de Malavane et Hulene qui présentent une caractéristique commune dans la région sud du Mozambique: dans les familles à bas revenu, rurales ou périurbaines, une bonne proportion d'entre elles voit la femme devenir chef de famille en l'absence de l'homme parti travailler en Afrique du Sud. Cependant la contrainte principale réside dans la non-reconnaissance de ce fait; les femmes ont donc la responsabilité de la maintenance sans l'autonomie de la prise de décision et sans accès aux ressources.

Les femmes seules, veuves ou mères célibataires, participent volontiers aux projets communautaires pour améliorer les conditions de vie, ceci sur une base bénévole, après leur journée de travail.

Certains enjeux sont communs aux trois régions énumérées:

- Les femmes apportent des solutions quotidiennes et créent des liens entre zones rurales et périurbaines à travers la production agricole de subsistance, qui constitue $70 \%$ de la production agricole totale du pays. Ceci n'est pas reconnu officiellement comme une production féminine, mais globalement comme "un secteur de production familiale".

- Les hommes et les femmes des zones périurbaines sont les victimes les plus affectées par le plan d'ajustement structurel. Ils se trouvent dans des situations de vulnérabilité, telles la violence et l'insécurité générale, qui aboutissent à des phénomènes sociaux de marginalité.

- Parallèlement, pour la même raison de lutte pour la survie, des réseaux de solidarité entre groupes de la même région apparaissent comme une stratégie sociale et introduisent des changements dans les relations entre hommes et femmes. 
Avec ces réflexions, formulées à un niveau encore bien superficiel, nous espérons avoir contribué à une meilleure compréhension de la modification des rôles de genre dans des situations de transition. Cela pourra peut-être contribuer à l'évolution des relations de genre et à une nouvelle approche politique du développement alors que jusqu'ici celui-ci est imposé à des pays d'une profonde diversité, sans prendre en compte leurs approches, leurs connaissances et leurs expériences. 


\title{
DIVISION SEXUELLE DU TRAVAIL ET GESTION DE L'ENVIRONNEMENT: LA VALORISATION DE LA FEMME ${ }^{1}$
}

\author{
KADIDIA TALL
}

\section{INTRODUCTION}

"Femmes, Villes et Environnement" constitue un vaste sujet, surtout lorsqu'on l'examine dans le contexte de Ouagadougou. En effet, les domaines suivants font partie intégrante de l'environnement urbain conditionnant la vie des citadins hommes aussi bien que femmes:

- la santé et l'hygiène avec l'approvisionnement en eau potable, la lutte contre les insectes dont les anophèles vecteurs du paludisme, l'équipement en latrines;

- l'assainissement par la collecte des déchets solides des ménages et des industries, par l'évacuation des eaux de pluies et des eaux usées;

- la circulation routière avec surtout un très grand nombre de cyclomoteurs se déplaçant trop librement et à l'origine de nombreux accidents;

- le chômage de très nombreux jeunes, anciens élèves et étudiants, la délinquance juvénile accentuée par la drogue, la prostitution;

- la pollution par les gaz d'échappement des véhicules divers et des usines, la pourriture des matières organiques et des eaux sales;

- la végétation assurant à la fois la purification de l'air, l'embellissement des maisons et des rues.

Parmi ces domaines nous allons en choisir un certain nombre sur lesquels les femmes, en tant qu'entité sociale, agissent quotidiennement pour sauvegarder et améliorer la qualité de la vie, ceux au niveau desquels on peut distinguer le rôle et le travail des femmes et des hommes. Pour que l'image soit aussi représentative que possible, nous avons choisi trois exemples qui nous paraissent des échantillons valables:

1. Nous voudrions adresser nos remerciements aux personnes et institutions de Ouagadougou pour leur gracieux concours, plus particulièrement à Mme Joséphine OUEDRAOGO du Bureau ARC et à M. Dieudonné Francis DISIBE de l'ONAT. 
- l'Association "Lagem Yam" qui utilise des charrettes à traction asine pour la collecte des ordures ménagères à très bas coût;

- les animatrices du secteur 7 qui développent la construction de latrines, tant au niveau familial que collectif;

- l'amicale de la SOCOGIB qui fait face aux dangers de la circulation, au besoin d'espace vert pour les loisirs et à la nécessité de solidarité entre voisins devant constituer une communauté unie.

Après une brève description de la ville de Ouagadougou nous traiterons du travail et du rôle des femmes et des hommes, puis de l'enjeu que constitue l'environnement pour valoriser la femme; enfin des conclusions et propositions seront données en guise de thème de recherches-actions.

\section{LA VILLE DE OUAGADOUGOU}

Ouagadougou, capitale du Burkina Faso, est située en zone sahélo-soudanienne, au coeur de l'Afrique de l'Ouest. Son climat est caractérisé par une longue saison sèche de sept mois et une saison pluvieuse s'étalant de mi-mai à mi-octobre. Les températures moyennes varient selon les saisons de 17 à $39^{\circ} \mathrm{C}$, la pluviométrie moyenne est de $700 \mathrm{~mm}$.

Ouagadougou est une des plus anciennes villes du Burkina Faso. Bien avant la conquête française (1896), elle était la capitale du royaume mossé de Wogodogo; en 1892 Binger estimait sa population à 5000 habitants. C'est surtout après l'indépendance du pays que la ville connut une explosion démographique étonnante: de 60'000 habitants en 1960, elle a passé à 634'479 soit 322'125 hommes et 312'354 femmes en 1991. Son taux de croissance est estimé à 9,3\% par an en 1992.

La population du point de vue de l'emploi mène des activités très variées dans les secteurs publics et privés. Ouagadougou étant la capitale du pays, les fonctionnaires sont nombreux $(41 \%)$.

Avec l'explosion démographique, l'Etat, les institutions privées et les services municipaux sont incapables de faire face aux besoins de la population tels que l'assainissement, le logement, les loisirs, la sécurité routière.

La ville de Ouagadougou, commune de plein exercice, est administrativement découpée en cinq arrondissements composés de 30 secteurs.

Les quartiers du centre ville (arrondissement de Baskuy) abritent les ministères et les services centraux, les ambassades, les maisons de commerce, les établissements financiers, les hôtels et restaurants "chics", les habitations de gens de toutes conditions sociales. C'est là qu'on trouve des quartiers plus structurés où vivent de hauts cadres et des personnes riches; ces quartiers sont beaux et ont peu de problèmes d'environnement dans l'ensemble.

En effet, l'arrondissement de Baskuy dispose de 115 bacs à ordures régulièrement enlevés par des camions qui opèrent automatiquement; de même, certaines parties de cet arrondissement connaissent pendant les périodes chaudes de l'année (mars, avril) des sensations de micro-climat plus frais à cause des nombreux arbres, des fleurs, du style de construction, et on y respire mieux. 
Ailleurs, et plus particulièrement dans les zones périphériques, les hommes et surtout les femmes connaissent de réelles difficultés pour avoir un cadre de vie agréable. Aussi les habitants plus ou moins aidés des autorités publiques et des services privés recherchent-ils des solutions pour faire face aux contraintes qui se présentent à eux.

Ces contraintes ou obstacles sont:

- L'absence ou le mauvais état de latrines faisant que certaines rues ou espaces verts sont devenus des lieux d'aisance soumettant ces quartiers au péril fécal (avec risque de propagation de l'amibiase, l'ankylostomiase, la fièvre typhö̈de, le choléra...).

Une enquête récente a permis d'établir les statistiques suivantes de répartition des ménages selon les types de lieux d'aisance à Ouagadougou:

- WC avec chasse d'eau

- Latrine privée

- Latrine commune

- Autres
$3,4 \%$

$55,5 \%$

$35,2 \%$

$5,9 \%$

- A certains endroits de la ville, le manque d'assainissement et l'absence de puisards et de caniveaux font que les eaux usées sont déversées dans les rues; pendant la saison des pluies de nombreuses maisons et des rues sont inondées; il n'est pas rare de voir des maisons construites en simples briques de terre s'effondrer.

- Les dépôts d'ordures ménagères dans les rues ou dans les cours rendent l'environnement désagréable pour les habitants.

- Les maisons d'habitation, selon les revenus des habitants, sont généralement regroupées en quatre catégories : les maisons en "banco" ou maisons en briques de terre, les maisons en "banco amélioré" qui utilisent des couvertures de tôles ondulées et dont les sols sont cimentés, les maisons en dur ou en matériaux définitifs très durables qui utilisent du ciment, des briques cuites, des carreaux en faïence, et les semi-dur dont les murs sont en "banco", les fondations en dur et le revêtement en ciment.

Les catégories de maisons en "banco" et "banco amélioré" correspondent aux habitations de ménages à bas revenus; aussi conçoit-on aisément que ce sont elles qui connaissent les plus grandes difficultés en matière d'assainissement.

Les stastistiques en la matière sont les suivantes:

- maisons en "banco"

- maisons en "banco amélioré"

- maisons en dur

- semi-dur

- maisons en paille

- autres
$35,2 \%$

$30,8 \%$

$20,0 \%$

$12,7 \%$

$0,5 \%$

$0,8 \%$

- Les espaces verts (ou vides) non encore aménagés qui sont réservés lors des lotissements ont été prévus pour recevoir des constructions d'utilité collective soit sociales (école, dispensaires, maternité, jardins publics, etc.) soit économiques (marchés, boutiques, services divers). 
Cependant, alors que les parcelles sont mises en valeur par la construction de maisons d'habitation, ces espaces réservés faute de moyens financiers collectifs attendent et constituent des parcelles vides ça et là au sein des quartiers, devenues progressivement des dépotoirs d'ordures, lieux d'aisance, garages.

En plus de ces contraintes ou obstacles appartenant au domaine de l'assainissement, il convient de mentionner le comportement destructeur vis-à-vis des arbres dont on coupe les branches pour les feuilles ayant des utilisations diverses; les fruits sont cueillis alors qu'ils ne sont pas mûrs. Ce sont le plus souvent les arbres du domaine public qui sont victimes de ces pratiques, bien que cela soit interdit. Les acteurs sont bien sûr généralement des enfants.

Dans cette ville aux graves problèmes d'environnement, il conviendrait de voir quels sont le travail et le rôle des hommes et des femmes.

\section{LE TRAVAIL ET LE RÔLE DES FEMMES ET DES HOMMES}

\section{Le rôle de la femme}

En mooré (langue des mossé) il est dit: "Pag la yiri": le femme c'est le foyer. La société lui assigne les rôles suivants: alimentation et entretien de la famille, éducation et santé de l'enfant, création et maintien d'un environnement sain et agréable.

Ainsi la femme est chargée de l'environnement dans lequel pratiquement tous les travaux sont effectués par elle:

- nettoyage des ustensiles de cuisine, lavage des habits, balayage de la maison, de la cour et du périmètre de la concession bordant la rue;

- collecte et vidange des déchets solides (ordures ménagères) et liquides (eaux usées);

- éducation des enfants à l'assainissement et à l'entretien de l'environnement: cette éducation est surtout contraignante pour les filles qui aident leur mère et apprennent ainsi tout ce qu'elle fait. Ces dernières années, dans quelques familles, les garçons bénéficient de la même éducation; ils pourront aussi contribuer à améliorer l'environnement.

\section{Le rôle de l'homme}

Il est le chef de famille; quand il le peut il procure l'argent nécessaire pour l'acquisition des biens servant à la femme : eau, savon, balais, ustensiles divers et de quoi payer les taxes de collecte des ordures. Signalons cependant que 11,1\% des femmes sont chefs de ménage.

Le creusement de fosses pour la construction de latrines ou de puisards est fait par les hommes. Les femmes et les enfants (garçons et filles) les aident. Lorsqu'ils en ont les moyens, ils payent une main-d'oeuvre spécialisée pour faire ces constructions. 
Un des rôles qui revient également à l'homme est la plantation d'arbre; en effet, en tant que propriétaire de la concession, il choisit l'essence et l'emplacement, creuse et plante. Ensuite, l'entretien de l'arbre, notamment sa surveillance et son ar rosage, revient à la femme.

\section{L'environnement collectif}

Ce domaine est constitué par les rues, les marchés, les dispensaires, les écoles, les "espaces vides", etc. Dans la plupart des cas, ce sont les hommes qui assurent l'assainissement de ces lieux en tant qu'employés de la municipalité, de l'Etat ou d'entreprises privées.

La femme est impliquée mais moins que l'homme; comme le travail se trouve valorisé par un revenu monétaire, ce sont surtout les hommes qui bénéficient des emplois pour l'assainissement, l'embellissement et la sensibilisation pour un cadre de vie meilleure.

A titre d'exemple, à l'ONASENE (Office National de Nettoyage et d'Embellissement), le service de nettoyage comptait 62 femmes et 90 hommes en janvier 1995. La présence des hommes semble se justifier par la nécessité de bras forts pour les travaux durs; c'est également ce qui est avancé pour exclure les femmes du service de collecte des ordures qui n'en compte aucune; en effet il faut conduire les machines et soulever les barriques remplies d'ordures, et cela ne conviendrait pas à leur condition féminine.

Les responsabilités dans les travaux liés à l'environnement au niveau familial et collectif relèvent des hommes. Ainsi en janvier 1995, sur 13 marchés de nettoyage, soit 13 équipes de travail, l'ONASENE comptait 3 femmes contre 10 hommes chefs d'équipe, bien que les femmes soient beaucoup plus nombreuses dans les équipes.

Malgré le travail et le rôle joué par les femmes et les hommes, les besoins en matière d'assainissement et d'amélioration du cadre de vie sont très loin d'être satisfaits. Face à cette situation, des initiatives communautaires ont vu le jour dans les secteurs 7, 10 et 13; leur incontestable succès en fait un modèle pour les autres secteurs de Ouagadougou. Fait remarquable, ce sont les femmes qui constituent la base de ces initiatives.

\section{LA COLLECTE DES ORDURES MÉNAGÈRES}

PAR LES COMMUNAUTÉS DE BASE À WOGODOGO (quartier du secteur 10)

Il s'agit d'un projet-pilote du Centre Régional pour l'Eau Potable et l'Assainissement à faible coût (CREPA) et de l'Institut Africain de Gestion Urbaine (IAGU). Les institutions sont parties du principe que si les populations sont organisées et appuyées, elles peuvent trouver des solutions à leur problème d'environnement. La collecte des ordures est faite avec des moyens non conventionnels; la finalité est l'amélioration de la santé des populations par la prise en charge de la salubrité et de l'hygiène du milieu par les habitants eux-mêmes.

Wogodogo a été choisi en raison du faible revenu de la population ; le quartier compte 3000 ménages et une population totale de $25^{\prime} 000$ habitants. 
La collecte des ordures est assurée par les femmes de l'Association "Lagem Yam" (Unissons nos intelligences) qui compte 12 membres. Des dispositions ont été prises dans les domaines de la communication, de la gestion financière et des réalisations techniques pour rendre efficace l'entreprise: salaires, location d'un local, engagement de personnel subalterne, recensement des abonnés, formation en technologies appropriées, en gestion financière et en éducation pour la santé; celles-ci respectent le niveau économique et culturel de la communauté de base constituée par les ménages. Actuellement 850 ménages sont abonnés à l'Association.

Ainsi les moyens de transport des ordures ménagères sont des charrettes tractées par des ânes; le matériel de collecte des déchets solides est constitué par des râteaux, fourches, pelles, seaux, balais, etc. Les femmes revêtent des équipements de protection: blouses, gants, bottes, masques à gaz.

Après douze mois, on constate que la rentabilité économique est atteinte et que les femmes ne ressentent aucune gêne à enlever des ordures qui ne sont pas les leurs. L'Association "Lagem yam" administre donc la preuve qu'avec une gestion saine et rigoureuse, une "micro-entreprise" peut être rentable.

L'exemple du succès de l'Association féminine "Lagem yam" est maintenant bien connu et suivi par d'autres quartiers de Ouagadougou.

\section{PARTICIPATION COMMUNAUTAIRE À L'AMÉLIORATION DE L'ASSAINISSEMENT \\ (secteur 7 de Ouagadougou)}

Ce projet pilote s'inscrit dans le cadre de la préparation du troisième projet de développement urbain intitulé "Projet d'Amélioration des Conditions de Vie Urbaine", financé par l'UNICEF et la Banque Mondiale. Le projet couvre les différentes composantes de l'assainissement relatives aux ménages: eaux usées et excréta, ordures ménagères, eaux pluviales.

Les animatrices constituent la cheville ouvrière de la sensibilisation, de la mobilisation de la population, hommes et femmes, de la formation en assainissement et de l'octroi aux femmes de crédit pour la construction des latrines.

Une équipe de quatre femmes, dont le chef est ingénieur sanitaire, assure la responsabilité de l'assainissement réalisé par la population, grâce aux animatrices du secteur formées par l'équipe appuyée par deux agents féminins du service de l'Action Sociale.

Les animatrices ont constitué des groupes de 10 à 12 personnes; cette organisation leur permet de préparer ensemble les messages qu'elles vont transmettre, chacune à son tour, à 15 autres femmes du secteur. Chaque groupe d'animatrices est supervisé par l'une d'entre elles sachant lire et écrire en français ou en langue nationale (mooré). Présentement, 180 animatrices ont été formées.

\section{Volet ordures ménagères}

$11 \%$ seulement des ménages évacuent leurs ordures en payant (charretiers, entreprises). La plupart des autres s'en débarrassent comme ils le peuvent: rues, espaces verts, parcelles vides servent de dépotoirs d'ordures ménagères; quelques-uns enfouissent ou brûlent les ordures. 
Des enquêtes sur le mode d'évacuation des ordures ménagères ont permis d'établir les stastistiques suivantes:

- utilisent une poubelle

- jettent sur un tas d'immondices

- jettent dans une fosse

- utilisent un bac à ordures

- jettent dans une décharge publique

- autres

$$
\begin{array}{r}
26,6 \% \\
46,3 \% \\
6,8 \% \\
1,8 \% \\
9,8 \% \\
8,7 \%
\end{array}
$$

Dans le cadre du projet, l'assainissement des lieux (concessions et rues) est réalisé par les femmes par le balayage et le ramassage des ordures qui sont ensuite soit vidées dans des bacs dont l'enlèvement est assuré par la Division Economique du Haut Commissariat, soit directement enlevées par Express Coopérative du Faso (ECOFA).

L'enlèvement des ordures est payé $500 \mathrm{~F}$ CFA par mois lorsqu'il a lieu à la porte de la concession ou 350 F CFA lorsque les ordures sont déposées au bac. Ce sont les femmes ou leur mari qui payent cette redevance.

\section{Volet crédit latrines}

Celui-ci est accordé exclusivement aux femmes formées qui désirent en construire à domicile. Aussi, sur les 180 femmes, 67 ont entrepris d'avoir des latrines décentes et fonctionnelles, car en matière d'évacuation des excréta $14 \%$ des concessions sont dépourvues de latrines et $80 \%$ ont des latrines traditionnelles sans système de ventilation et connaissent des problèmes de vidange.

Ces femmes ont amené les hommes à accepter que ces latrines "modernisées" soient construites dans leurs cours (ils en sont responsables), qu'ils les accompagnent auprès de l'équipe d'animation pour les inscriptions et s'engagent à rembourser totalité ou partie du crédit de 19'500 F CFA payable en dix mois.

Le projet pilote, fondé sur la mobilisation des femmes par les femmes (animatrices), dirigé par une équipe de femmes, est une réussite qui pourra servir d'exemple à d'autres secteurs de Ouagadougou. Aussi les efforts déployés méritent-ils d'être poursuivis.

\section{L'AMicAle Des Femmes de la SOCOGIB}

La cité de la SOCOGIB Zone du Bois constitue un quartier moderne du secteur 13; toutes les maisons sont en dur et les rues bitumées; de ce fait, il y a très peu de problèmes d'assainissement; par contre l'embellisement, les espaces verts, les loisirs, la sécurité des enfants, la vie sociale constituent les préoccupations des habitants.

L'Amicale qui regroupe une centaine de femmmes a à son actif:

- l'aménagement d'un espace collectif avec des banquettes, des aires de jeux, un hangar, qui sert aussi bien aux enfants pour s'amuser en toute sécurité qu'aux hommes et aux femmes pour leurs réunions et leurs distractions; des kermesses y sont organisées; 
- la mise en place de panneaux de circulation routière qui assurent une plus grande sécurité pour les enfants ;

- les manifestations de solidarité à l'occasion des évènements de la vie, qu'ils soient heureux (baptêmes, mariages) ou malheureux (maladies, décès);

- la plantation et l'entretien d'arbres par les femmes et les enfants du quartier: les cotisations permettent de payer des manoeuvres pour creuser et planter; la surveillance et l'ar rosage sont assurés par les enfants et les femmes.

Ainsi les femmes font face aux besoins de la collectivité: sécurité des enfants, loisirs des adultes, convivialité entre les habitants d'un même quartier. C'est là une autre facette importante de la relation femmes-villes-environnement.

En conclusion de cette deuxième partie nous disons que l'assainissement et l'organisation des espaces collectifs relèvent de la compétence des communautés de base, des secteurs et des arrondissements qui doivent mobiliser les moyens pour permettre à la femme de jouer son rôle et d'améliorer son cadre de vie au profit de toute la collectivité urbaine.

La mise en valeur et la modernisation de la ville de Ouagadougou nécessitent de gros moyens financiers et humains, face à une croissance démographique galopante. Ces moyens sans l'aide de la communauté internationale ne peuvent être mobilisés par les collectivités locales décentralisées. C'est pourquoi le problème demeure complexe.

\section{L'ENVIRONNEMENT URBAIN, ENJEU ET BASE FONDAMENTALE POUR VALORISER LA FEMME}

Dans ce qui précède on constate le rôle éminent des femmes tant au niveau du ménage que de la collectivité.

Pour la collectivité, l'environnement est un enjeu des plus important pour le développement économique et social. En effet, sans un environnement sain et agréable il ne peut y avoir de développement durable.

Le rôle et le travail si importants des femmes doivent constituer une base fondamentale et un tremplin pour leur valorisation. Aussi, il conviendrait d'amener la collectivité à reconnaitre la juste valeur du travail de la femme en matière d'environnement par une sensibilisation voire une éducation surtout au niveau de la conception traditionnelle du peu de valeur des activités d'assainissement; il faudrait également que la femme soit associée au lotissement des quartiers, au plan de construction de la maison familiale.

En ce qui concerne plus particulièrement l'assainissement, dont la femme a la charge, il faudrait remédier au fait qu'elle n'a ni pouvoir, ni moyens pour organiser les espaces collectifs dans l'intérêt des populations et particulièrement des enfants. 
Pour illustrer cette nécessité de la valorisation des activités d'assainissement, deux anecdotes racontées par les femmes qui collectent les ordures ménagères sont à citer tant elles sont éloquentes:

- une femme collectant des ordures a été reconnue par une de ses copines qui la connaissait comme marchande de beignets; elle lui a demandé si elle ramassait les ordures parce que son commerce avait périclité. Cette copine ne pouvait pas donner plus de valeur au ramassage des ordures qu'à la vente des beignets;

- une autre femme collectant des ordures ayant rencontré une de ses copines, celle-ci comme par compassion a dit que "Dieu nous aide à bien finir". Sous-entendant que collecter des ordures ménagères était la pire tâche qu'on pouvait avoir dans la vie.

Il conviendrait donc de sensibiliser les populations par une éducation civique véritable afin qu'elles valorisent ces travaux d'assainissement de l'environnement et, partant, les femmes qui les assurent en leur donnant une formation adéquate et une meilleure rémunération.

Au niveau des entreprises de la place assurant l'assainissement, ce serait rendre justice aux femmes que de leur donner la priorité de recrutement et une place de choix au sein de l'entreprise plutôt que de les marginaliser.

\section{CONCLUSIONS ET PROPOSITIONS}

Il est utile de faire valoir ici les idées forces, un ensemble de propositions, les plus simples et les plus pragmatiques possibles.

"Femmes, Villes et Environnement" dans le contexte de Ouagadougou a été examiné sous l'angle socio-économique, les aspects politiques et culturels ayant été laissés de côté. Trois exemples ont été donnés où les femmes ont pris en charge les actions nécessaires à l'amélioration du cadre de vie pour le rendre plus sain, plus sécurisant et plus agréable:

- Lagem yam dans le secteur 10 assure une grande partie de l'assainissement du quartier (Wogodogo) par le ramassage des ordures ménagères avec des moyens non conventionnels (charrettes tractées par des ânes).

- Le Projet Pilote de Participation Communautaire à l'Amélioration de l'Assainissement dans le secteur 7 , fondé sur les animatrices, arrive à mobiliser les femmes du secteur pour assurer l'enlèvement des ordures ménagères et l'évacuation des excréta par la construction de latrines.

- L'Amicale des Femmes de la SOCOGIB du secteur 13, où les femmes ont initié et réalisé des aménagements au profit de toute la communauté, crée ainsi une plus grande solidarité entre les habitants.

Dans ces trois cas il ressort que les femmes assurent le rôle que leur attribue la société. N'ayant pas beaucoup de pouvoir - la loi fait de l'homme le chef de famille et c'est lui qui est propriétaire de la maison familiale - ni autorité politique, administrative, coutumière ou religieuse, elles ont dû user de leur rôle et de leur travail pour obtenir la contribution des hommes en les amenant à prendre les décisions nécessaires, à débourser l'argent indispensable pour réaliser ce qu'elles ont fait, avec parfois la contribution des services de l'Etat ou de projets. 


\section{Propositions}

Les propositions qui suivent visent à l'amélioration de l'environnement au niveau de la ville de Ouagadougou; elles se fondent sur les femmes en leur faisant une plus juste "place au soleil" en rapport avec leur éminente contribution à rendre le cadre de vie plus sain et plus agréable pour faciliter l'épanouissement des enfants et de l'ensemble de la famille.

Ces propositions constituent une esquisse de programme de Recherches-Actions composé de projets dont les coûts ne sont pas estimés. Ce programme comprend quatre volets intégrés:

- assainissement,

- ville verte,

- écoute, échanges, loisirs,

- emplois.

Ces quatre volets coexisteront toujours, l'importance de chacun d'eux variant selon les besoins réels du milieu déterminés par la Recherche-Action qui devrait être participative et donner une place de choix aux femmes.

Le programme couvrirait toute la ville de Ouagadougou, c'est-à-dire les cinq arrondissements divisés en 30 secteurs, chacun des secteurs étant constitué par des quartiers qui souvent plongent des racines profondes dans l'histoire plus que séculaire de la ville.

Il faut agir au niveau du quartier, donc de la communauté de base. Des recherches-développements pourraient être réalisées en étroite relation avec les associations féminines et déterminer les actions à mener dans les quatre domaines retenus.

\section{Projet du volet assainissement}

Les femmes seraient sollicitées à toutes les phases du projet: élaboration, réalisation, suivi-évaluation. Le projet s'intéresserait aux aspects construction de latrines et de puisards, enlèvement des ordures ménagères. Les voies et moyens de réalisation feraient une place importante aux femmes en tenant compte de leurs conditions socio-culturelles: niveau de revenus et d'instruction notamment. Toutes les expériences actuelles seraient prises en compte, notamment l'utilisation de moyens non conventionnels.

La construction de latrines et de puisards devrait offrir une gamme variée en fonction des possibilités financières des ménages qui pour raient bénéficier de crédits à des conditions souples, voire des subventions partielles. De plus, pour les personnes ne pouvant disposer d'un domicile, il conviendrait de prévoir des latrines publiques utilisées à des prix sociaux.

Les matières organiques contenues dans les ordures ménagères et les excréta devraient, si possible, être transformées en engrais. La production maraîchère autour de Ouagadougou, où les femmes sont actives, pourrait les utiliser sous forme de compost.

\section{Projet du volet ville verte}

Ce projet pour rait mobiliser les femmes pour planter et entretenir le maximum de végétaux. Cela nécessite de multiples actions, notamment la sensibilisation et la formation sur l'importance des plantes pour l'homme: 
- elles protègent le sol contre l'érosion, certaines (les légumineuses) le fertilisent;

- les arbres donnent de l'ombre, une ombre si précieuse dans le contexte très ensoleillé et chaud de Ouagadougou qui abriterait de nombreuses activités: travaux domestiques (cuisine, lessive, pilage du mil), réunion sous l'arbre à palabre, petit commerce;

- les plantes fourragères permettent de produire les denrées alimentaires essentielles telles que la viande (lapin, chèvre, mouton, etc.) et le lait (chèvre, vache);

- les plantes médicinales telles le néré, le tamarinier, l'eucalyptus, etc. sont très utiles, surtout que la grande majorité de la population a un revenu très faible;

- les plantes embellissent l'environnement, essentiellement par leurs fleurs.

Pour chaque catégorie ci-dessus citée, on pourrait choisir des essences adaptées aux conditions climatiques de Ouagadougou, surtout celles qui sont peu exigentes en eau.

Une recherche-développement conduirait à estimer les besoins de plants aussi bien pour les concessions que pour les places publiques et les rues. Ainsi, des pépinières seraient créées, entretenues, gérées par les femmes.

La recherche-action permettrait de déterminer, en étroite relation avec les femmes, la taille de chaque projet, les voies et moyens de sa réalisation, notamment le coût et le mode de financement ainsi que les lieux de plantation et le mode de financement.

\section{Projet du volet écoute, échanges, loisirs}

Ce volet, bien qu'intéressant l'ensemble de la communauté de base, serait davantage destiné aux femmes et aux jeunes.

Dans le cadre de l'IEC (Information, Education, Communication), des lieux seraient aménagés, équipés pour faciliter l'écoute et l'échange, entre les jeunes surtout afin qu'ils puissent aisément s'informer sur de multiples sujets, l'environnement en particulier.

Loisirs: il s'agit ici d'espaces de jeux ouverts ou fermés, sportifs ou non. Les jeux peu exigeants en espace, praticables par les deux sexes, seraient préférés (basket-ball, volley-ball, etc.). De plus, des jeux de société seraient offerts: cartes, jeux de dames.

La recherche-développement définirait les besoins réels de la population en tenant surtout compte du contexte culturel de la communauté de base. Rappelons qu'en tout temps l'homme a accepté de dépenser pour s'amuser.

\section{Projet création d'emplois}

Il ne s'agit pas d'un volet particulier mais d'une conséquence des autres volets (assainissement, ville verte, écoute-échanges-loisirs).

Ces volets devraient être conçus de manière à générer des emplois pour les femmes. On trouve au niveau des communautés de base plusieurs possibilités pour réaliser des activités, notamment le bénévolat et les emplois salariés. Ces derniers devraient si possible être permanents et destinés aux femmes. 
Les avantages de la création d'emplois pour les femmes sont essentiellement de deux ordres:

- une formation professionnelle qui leur permet de faire un travail dont la qualité est nettement supérieure à celle produite par le bénévolat;

- un apport de revenus qui leur donnent une certaine aisance et surtout une relative indépendance financière.

Bibliographie:

Assemblée générale des villes jumelées du Burkina Faso, du 28 au 30 juillet 1993 à Kaya.

Thème: "Jumelage, coopération et envi-ronnement".

Atlas Jeune Afrique (Burkina Faso), Paris, Editions Jeune Afrique, 1993.

BARO Joséphine, BAKYONO Valentine, KERE Aminata, ZAGRE Rosine, Projet pilote de partici pation communautaire à l'amélioration de l'assainissement secteur 7, Onagadougou. Bilan et perspectives, Ouagadougou, Ministère des travaux publics, de l'habitat et de l'urbanisme du Burkina Faso/UNICEF, juin 1994.

Femmes et santé $\mathrm{n}^{\circ}$ 5, novembre 1993 (Bulletin semestriel d'information sur la santé des femmes en Afrique sub-saharienne: "La division sexuelle des tâches et des responsabilités et son impact sur la santé des femmes"), BP 724, Yaoundé.

Haut Commissariat du Kadiogo Ouagadougou, Carte d'identité de la province du Kadiogo (Réactualisation 1992), Programme Mieux Vivre à Ouagadougou, 1994.

Info CREPA n ${ }^{\circ} 6$ octobre, novembre, décembre 1994 (Revue trimestrielle du Centre Régional pour l'Eau Potable et l'Assainissement à faible coût - dossiers: Collecte des ordures par les communautés de base de Wogodogo), 03 BP 7112, Ouagadougou.

INSD, Enquête démographique 1991: Etat de la population, Habitat et ménage, Ouagadougou, INSD, 1991. 


\title{
DE LA NECESSITE DE REINVENTER DE NOUVEAUX RAPPORTS HOMMES-FEMMES EN AFRIQUE AUJOURD'HUI
}

\author{
AMINATA TRAORE \\ FATIMA MEITE
}

Ce libellé - Ville, genre et environnement - renvoie à l'un des défis majeurs auxquels le Tiers Monde est actuellement confronté. Tentons d'en cerner les contours en Afrique subsaharienne.

Cette région du monde est caractérisée, en cette aube du XXIe siècle, par une crise sans précédent, aux causes multiples, dont les principaux aspects se laissent saisir d'emblée dans les villes: chômage et sous-emploi malgré un secteur informel particulièrement dynamique, une population jeune, l'insuffisance des infrastructures et équipements de base, le manque d'eau potable, de source d'énergie domestique et les difficultés d'évacuation des ordures ménagères. Les femmes y sont particulièrement confrontées à une pauvreté croissante.

Il en est ainsi parce que, contrairement à l'Europe où l'histoire de l'urbanisation se confond avec celle de l'industrialisation, les villes africaines, qui sont de création récente, ne sont pas des pôles de développement, créateurs et promoteurs d'emplois.

Elles absorbent, saisonnièrement ou définitivement, l'essentiel des forces vives de la campagne et hypothèquent ainsi, dans une large mesure, les chances de développement de l'arrière-pays et surtout de l'agriculture, qui constitue l'épine dorsale des économies nationales. Mais les villes africaines n'en sont pas moins dynamiques parce que tirées par des acteurs sociaux qui y développent chaque jour leurs propres stratégies et, en fait, réinventent les villes à leur manière. Les ménages en sont devenus des microcosmes où hommes, femmes adultes et jeunes développent de nouveaux types de comportements et des rapports interpersonnels qui sont autant de réponses à un projet de société improvisé, non concerté et non explicité.

Cette situation revêt des dimensions qui ne se prêtent pas facilement à l'analyse, tant les changements sont rapides et complexes. Les variables telles que l'âg e, l'ethnie, la religion, l'origine géographique et le niveau d'instruction influent sur la manière dont les rapports hommes-femmes se structurent.

\section{HOMMES ET FEMMES EN VILLE}

Les termes de la problématique de l'urbanisation ont considérablement changé en Afrique subsaharienne ces dix dernières années. Dans les années 60 et 70 , on déplorait le caractère déséquilibré des investissements entre villes et villages, l'exode rural qui en résultait, la délinquance, etc. 
La situation s'est détériorée davantage tout le long des années 80 avec la mise en oeuvre des Programmes d'ajustement structurel. La compression de l'emploi salarié, le gonflement du secteur informel, la détérioration des infrastructures et équipements de base, voire leur inexistence, le développement de l'habitat spontané en zone périurbaine... sont autant de caractéristiques des villes de la fin des années 90 en Afrique. Cet ensemble de facteurs a agi sur les rapports hommes-femmes, pas nécessairement dans le sens d'une plus grande exclusion des femmes mais aussi par leur entrée dans des secteurs nouveaux et parfois le renforcement des liens de solidarité entre conjoints. En réalité, et on ne le souligne pas assez, la pauvreté urbaine touche autant les chefs de famille, qui assistent à une baisse de leur pouvoir d'achat (synonyme d'autorité), voire à la perte pure et simple de leur emploi. Les femmes sont alors condamnées à subir seules les contrecoups des réformes économiques en termes de participation financière au coût des services urbains: approvisionnement en eau potable, enlèvement des ordures ménagères, soins de santé.

Les explosions socio-politiques qui embrasèrent la plupart des capitales africaines entre 1990 et 1993, et qui débouchèrent sur une certaine ouverture politique, appelée démocratie, étaient d'abord l'expression du mécontentement des gens de la ville, hommes, femmes et jeunes. La majorité des manifestants étaient des citadins et surtout des citadines excédées par trente années d'essais du développement, dont dix années d'ajustement, sans résultats probants. Ce sont, en plus des syndicalistes, des mères de famille et des élèves qui acculèrent les régimes politiques en place et prirent d'assaut les symboles du pouvoir: bâtiments publics et autres biens matériels mais aussi des personnes physiques.

La situation ne s'est pourtant pas améliorée avec l'aide du pluralisme politique. Au contraire, les 14 pays de la zone franc ont assisté à la détérioration de 50\% de leur pouvoir d'achat avec le changement de parité survenu en janvier 1994.

L'un des traits particuliers de la situation des villes africaines et des rapports homme et femme qui y ont prévalu est la prise en charge du coût social des réformes économiques et monétaires par les femmes dans les couches sociales défavorisées. Les dynamiques urbaines qui s'instaurent révèlent, d'une part que les rôles féminins et masculins ne sont pas systématiquement conflictuels dans les sociétés non industrialisées d'Afrique, et d'autre part que les femmes jouissent, dans le contexte de crise qui prévaut, d'une marge de manoeuvre importante.

Elles ont la latitude d'exercer leurs talents et leur créativité surtout dans le secteur informel qui échappe pour l'instant à la comptabilité nationale. Et quand elles parviennent à s'insérer dans des secteurs initialement contrôlés par les hommes, elles sont confrontées à des contraintes qui sont plus liées à l'incapacité des Etats à repenser les politiques urbaines qu'à une inégalité de chance entre homme et femme, comme l'atteste l'expérience d'un groupe de femmes qui sont exploitantes de matériaux de construction.

\section{DÉFIS NOUVEAUX ET CHANGEMENTS DE RÔLES SOCIO-ÉCONOMIQUES}

Il convient d'étayer les points de vue ci-dessus mentionnés à la lumière de faits tels que les femmes qui sont en situation l'interprètent elles-mêmes. Le cas des exploitantes de matériaux de construction, mentionné plus haut, est particulièrement riche d'enseignements que nous résumons ainsi: 


\section{La pauvreté}

"Je me suis lancée en 1971 dans ce secteur initialement réservé aux hommes à cause de la pauvreté. Par la suite trois autres femmes m'ont emboité le pas. Mais nous avons été trahies par les politiciens qui se sont servis de nous en exhibant notre cas lors des cérémonies officielles sans jamais rien faire pour nous. A présent, nous sommes décidées à nous organiser sans rien attendre d'eux."

\section{Rapports hommes-femmes}

"Nous avons affaire, en plus de nos maris, aux hommes qui travaillent dans le même secteur que nous. S'agissant des premiers, disons que la plupart d'entre nous sommes chefs de famille parce que veuves ou divorcées. Les autres ont des maris chômeurs qui ne leur posent pas de problèmes particuliers même si parfois ils déplorent le fait qu'on ait à côtoyer d'autres hommes à longueur de journée. Mais ces hommes que nous côtoyons dans le cadre de notre travail ne nous regardent pas. Parce que nous regardons tous dans la direction de l'argent... Au début nous avions même créé une coopérative avec les hommes. Mais les tensions commencèrent à se multiplier entre eux et nous à cause de la crise: il y a moins de travail. Alors certains nous rappellent que nous n'avons pas à nous mesurer à eux et que notre place est dans nos foyers."

\section{Besoins ressentis}

"Aucune d'entre nous n'est instruite. Nous avons appris, pendant près de vingt ans, à nous battre seules; mais nous avons besoin d'être éclairées sur bien des aspects de ce secteur qui nous échappent."

\section{Contraintes familiales}

"Nous partons très tôt le matin en confiant la garde des enfants à une bonne qui repart le soir sans attendre notre retour. Il arrive qu'en rentrant nous trouvions les enfants sortis, sans trop savoir où ils sont passés."

Perception des rapports entre hommes et femmes

"Ils (les exploitants masculins) sont des hommes et nous des femmes et notre pays a ses particularités. Si nous travaillons ensemble, hommes et femmes, nous trébucherons mais nous ne tomberons pas. Nous devons leur prouver l'envie de coopérer avec eux et non de les combattre."

Ces propos sont suffisamment clairs quant à la nature des défis nouveaux que les nouvelles catégories socio-professionnelles offrent et que les femmes urbaines se proposent de relever.

\section{LES EFFETS DE LA CRISE DU DÉVELOPPEMENT ET DE L'ENVIRONNEMENT}

Le développement, qui n'a pas tenu ses promesses en Afrique, a par contre réussi à démanteler les structures familiales et à détériorer considérablement l'environnement. La plupart des chefs de ménage sont des salariés de la Fonction publique, des ouvriers licenciés de quelques entre- 
prises publiques restructurées, de petits commerçants et des artisans plus ou moins ruinés par la crise de la dette.

Les systèmes de production dans lesquels ils s'étaient relativement bien intégrés avant la crise, ont donné lieu à une division sexuelle du travail qui est en pleine mutation.

En effet, en l'absence des opportunités que le milieu rural offre ou offrait (ressources forestières, terres, eau, bois de feu...), les rapports entre citadins et citadines ont été restructurés sur une base selon laquelle le chef de famille s'acquitte des responsabilités qui lui incombent dans les économies agraires mais en achetant, par exemple, du riz et du mil. Pendant ce temps, sa femme est déchargée de la fourniture des condiments et autres denrées qu'elle achète sur le marché avec la part du revenu de son conjoint qui est consacré à ce poste de dépense. Si bien qu'en ville, les hommes utilisent généralement l'expression "courir derrière le prix du condiment" pour traduire l'effort qu'ils doivent fournir pour assumer pleinement leur rôle de chef de ménage. Telle était la situation avant la crise des années 80.

En modifiant cette répartition des rôles entre hommes et femmes, les Programmes d'ajustement structurel fragilisent l'équilibre des familles. Un nombre croissant de femmes urbaines sont devenues des chefs de famille qui assument les rôles de l'un et de l'autre sexe.

Mais même dans les unions stables où les maris sont compressés ou partants volontaires de la Fonction publique, ce sont les femmes qui s'attellent, de plus en plus, à toutes les activités qui peuvent permettre de colmater les brèches. Elle supportent ainsi le coût social des réformes économiques et monétaires imposées par les institutions internationales de financement aux pays surendettés d'Afrique.

Les cadres analytiques, les méthodes et les outils de la recherche-action gagneraient à être repensés à la lumière de ces faits nouveaux. 


\title{
LES ACTIVITES FEMININES DANS L'ENVIRONNEMENT URBAIN
}

\author{
EMMANUEL NDIONE
}

\section{HisTORIQUE}

Dans notre institution (voir annexe), notre analyse a été de privilégier l'action en faveur des femmes, essentiellement à travers l'organisation familiale et, dans ce cadre, la maternité. Nos actions par rapport à la maternité ont comme objectif essentiel d'appuyer ces fonctions sociales à travers des interventions touchant l'économie familiale, le suivi de la croissance pondérale de l'enfant, la récupération nutritionnelle.

Disons tout de suite que nous avons projeté sur le fonctionnement familial nos propres clivages parce que nous nous sommes déterminés à partir d'une fonction et non de l'ensemble des fonctions jouées par les femmes au sein de la famille et dans le quartier.

Ces actions n'ont pas nécessairement abouti parce que l'objectif attendu consistait essentiellement à créer un trait d'union entre les femmes organisées dans les quartiers et les services officiels de santé, à travers la mise en place d'un réseau d'auxiliaires de santé. Ces auxiliaires devaiten assurer à terme, par le bénéfice du transfert des fonctions qui étaient les nôtres, le suivi des actions au niveau du quartier et assurer la liaison avec les postes de santé.

Conscients des limites de cette action, nous avons cherché à greffer aux activités de santé des projets générateurs de revenus. C'est un moyen d'intéresser la femme car, dans les quartiers populaires, c'est elle qui est au centre de la gestion de la famille. L'homme part toute la journée à la chasse et ne revient que dans la soirée. Quel que soit "le gibier" qu'il rapporte ou ne rapporte pas, la responsabilité concrète de l'entretien du foyer incombe totalement à la femme.

Nous nous sommes donc naturellement intéressés à ces activités et nous avons engagé des actions devant générer des revenus et développer une autonomie personnelle et familiale. Tout l'argent dont nous disposions avait pour but d'aider les femmes à mettre en place des activités économiques. Pour réussir, il nous fallait aider les femmes à prendre en compte une logique économique et comptable. L'assimilation des techniques comptables était vite apparue comme une nécessité.

Parallèlement, nous avons créé des systèmes institutionnels parce que les systèmes organisationnels locaux nous paraissaient fonctionner de façon moralement inacceptable. La position centrale de la présidente qui transposait dans le groupe sa fonction sociale nous gênait beaucoup. Cette présidente n'avait pas intégré la "culture démocratique". Elle était et voulait rester l'aînée sociale. Ses partenaires n'attendaient rien d'autre que cela.

Nous avons marqué notre réticence à appuyer cette fonction sociale, ce qui nous a amenés à détourner notre attention des associations en place et à encourager la constitution de nouvelles associations, plus aptes à assimiler "la culture du développement". 
En mettant les femmes en condition d'appliquer des techniques comptables et de fonctionner dans de nouveaux "enclos" sociaux, nous avons largement contribué à leur déstabilisation et favorisé l'insécurité de la famille. Rompre avec leur stratégie de polyactivités et se consacrer à une seule activité impliquait" pour les femmes de renoncer aux "socialités" coutumières.

Nous nous sommes rendu compte qu'à notre stratégie soi-disant d'autonomisation s'opposait celle des femmes qui consistait à développer des démarches de rattachement. Ce qui les intéressait dans leur rapport avec nous, c'était moins l'activité économique que l'opportunité d'une alliance avec chacun de nous et avec notre institution.

\section{L'APPUI AUX ORGANISATIONS EXISTANTES}

Dans un deuxième temps, au lieu de faire de la création institutionnelle, il nous a semblé plus important de légitimer les organisations existantes et de les appuyer. Cela supposait un autre pari: il fallait accepter le système de fonctionnement interne des groupes déjà existants. La mère et/ou la présidente n'étaient pas simplement celles qui devaient gérer une activité selon des principes démocratiques et égalitaires. La mère - ou la présidente - est l'aînée sociale accumulatrice et redistributrice. Son groupe c'est sa famille, les membres ses cadets. Dès lors l'important est de renforcer le système d'accumulation, de circulation de ressources et d'extension des réseaux relationnels. A l'opposé de notre démarche favorisant l'immobilisation d'importantes sommes d'argent et la mise hors des circuits sociaux, les femmes cherchent à accroître la vitesse et l'aire de circulation des ressources financières, relationnelles... Pour elles, la sécurité passe par la mise en perspective de toutes les ressources disponibles.

Une fois que cette démarche a été mise en évidence, ce qui nous paraissait essentiel ce n'était plus d'encourager un type d'activité quelconque mais d'appuyer la fonction de la circulation de l'argent, des ressources en nature, des relations humaines, favoriser des placements diversifiés en terme d'activités, de relations sociales. L'extension du réseau de relations est à la base de la sécurité recherchée par tous. Il ne s'agit plus d'autonomie isolée mais "d'allonomie", c'est-à-dire d'une autonomie fondée sur une relation de réciprocité.

On s'est ainsi mis en condition de rendre l'argent disponible pour une multiplicité de placements de toute nature, dans des domaines jadis sans lien aucun, l'économique, le sacrificiel. Beaucoup de personnes qui ont emprunté de l'argent ont financé des actes sacrificiels parce que leur rattachement aux forces divines et la recherche de complicité avec diverses composantes connues pour leurs vertus valorisantes pour l'environnement étaient devenus une condition de réussite de toute activité ou entreprise humaine. Lorsque les femmes entreprennent une activité de commerce, par exemple, elles placent dans leur étal des petits citrons qui ont pour fonction de valoriser la marchandise aux yeux de la clientèle.

Forts de tous ces enseignements, nous avons décidé d'encourager les activités initiées par les femmes elles-mêmes. Le décloison-nement des activités et des groupes identifiés a permis de dégager d'importantes sommes d'argent apportées par les différents sociétaires. 


\section{LA VALORISATION DES ACTIVITÉS FÉMININES}

Désormais les tiroirs ne sont plus étanches puisqu'un facteur économique comme l'argent peut financer une activité sacrificielle. Libérées de toute conditionnalité, les femmes sont devenues plus dynamiques et plus créatrices. Des réseaux d'échange se sont mis en place permettant la diffusion de toutes les expériences et la valorisation des expertises populaires féminines.

L'action sociale n'est donc plus le monopole d'organisations masculines et différentes questions touchant tout ou partie de la communauté sont traitées entre femmes. Un centre d'expertise populaire féminine a été créé et permet la mobilisation réciproque des compétences et la prise en charge des questions liées à la valorisation du point de vue féminin.

Mais, pour en arriver là, il faut modifier nos fonctionnements institutionnels. Il nous parait capital de réfléchir sur notre rôle et sur les discriminations et les hiérarchisations entre les points de vue masculins et féminins. Les lieux attribués ou réservés aux femmes et socialement reconnus ou admis par toutes et tous sont toujours définis en termes inessentiels ou périphériques.

Désormais, l'angle de lecture femme constitue pour nous une perspective de groupe social dont la prise en compte est indispensable pour notre société. Il faut donc que notre action puisse aider les femmes à s'assumer pleinement comme sujets historiques pour elles-mêmes, pour leur quartier, pour leur village...

comme c'est le cas pour de nombreuses employées de maison vis-à-vis de leur groupe ou communauté villageoise où elles se rendent régulièrement pour mener des actions.

\section{ANNEXE}

\section{HistoriquE DE L'ORGANISATION ENDA/TM}

ENDA/TIERS MONDE (Environnement et Développement du Tiers Monde) est une organisation à caractère associatif et à but non lucratif.

ENDA a fonctionné à partir de 1972, notamment avec l'appui du PNUE, comme programme de formation pour l'environnement, est devenu une association en novembre 1977, s'est transforrmé en juin 1978, à la suite d'une convention d'établissement signée avec la République du Sénégal, en une organisation internationale dont le siège est à Dakar.

ENDA/TM est l'un des tous premiers groupes qui, en Afrique, ont tracé très tôt la relation Environnement/Développement et parlé d'écosystème dans la perspective de la construction du futur.

\section{DESCRIPTION DES ORIENTATIONS ET DES ACTIVITÉS ENDA}

\section{Les objectifs entre autres}

- appuyer la recherche ainsi que les types de formation qui permettront de parvenir à un développement alternatif; 
- contribuer à l'engagement des intellectuels et des cadres dans la définition et la mise en oeuvre d'un développement au service du plus grand nombre.

\section{Les méthodes de travail entre autres}

En s'inspirant des actions de développement des groupes de base et de la lutte des peuples du Tiers Monde, ENDA recherche en permanence une méthodologie qui puisse satisfaire leurs besoins et leurs désirs d'indépendance:

- maintien d'un lien étroit entre action, réflexion et formation;

- échanges intensifs d'expérience et de techniques entre les différentes régions du Tiers Monde. ENDA, avec de nombreux partenaires, anime ainsi un réseau d'échanges et de réflexion dans l'ensemble du Tiers Monde. ENDA envisage une auto-planification des groupes de base en liaison avec des organisations régionales, nationales et internationales de déve-loppement.

\section{Les axes de travail prioritaire d'ENDA}

- repérage et réduction des disparités socio-spaciales, notamment des inégalités entre régions et à l'intérieur de celles-ci, ainsi que dans les milieux urbains;

- développement de la communication au niveau des groupes de base: relance des échanges dans les quartiers et villages, transmission des acquis culturels et des expériences, concertation sur les actions à entreprendre;

- aide aux peuples culturellement les plus menacés: transformation de la perception qu'en ont les autres peuples et appui à un développement qui respecte leur originalité; combat pour les droits des peuples et de l'Homme.

\section{QU'EST-CE QUE ENDA GRAF/SAHEL?}

ENDA GRAF/Sahel et Afrique de l'Ouest (Groupes Recherche-Action-Formation) est une antenne, dotée d'une autonomie juridique, de l'organisation internationale ENDA/Tiers Monde.

ENDA GRAF/SAHEL développe une méthodologie dénommée recherche-action-formation qui a pour but de renforcer les démarches populaires et de permettre aux populations d'améliorer l'analyse qu'elles font des situations, de rechercher des solutions appropriées, de mobiliser d'abord leurs propres ressources pour l'action, de négocier ou de rechercher les moyens de réaliser des actions, de s'organiser pour l'action, d'évaluer leur propre démarche et leurs résultats.

C'est ce processus qui est formateur. La recherche paysanne et populaire est au ras du sol et utilise essentiellement les moyens de commu-nication et de langage des populations.

ENDA GRAF/SAHEL couvre les régions du Sahel et de l'Afrique de l'Ouest et poursuit des objectifs à plusieurs niveaux qui sont:

- appuyer les démarches d'appropriation par les populations;

- favoriser l'émergence d'une expertise populaire et faciliter son emploi et son extension;

- mettre en relation les "groupes innovateurs" et les "experts" urbains ou ruraux en Afrique et entre l'Afrique et d'autres tiers-mondes, notamment l'Amérique latine, l'Asie et les Caraïbes ; - favoriser la mise en place de réseaux d'experts populaires et renforcer leurs pouvoirs de négociation et d'action. 
Des démarches privilégient trois dispositifs de communication interactifs:

La Recherche comme outil d'évaluation permettant aux projets d'améliorer leur approche des situations et l'appropriation des objectifs visés par les populations; analyse et évaluation des pratiques par les intéressé(e)s eux-(elles)mêmes; identification des personnes-ressources en vue de valoriser leurs capacités de recherche; échanges à partir d'évaluations d'actions, de confrontations d'expériences, de consultations réciproques ; expérimentation des solutions dans l'aménagement des terroirs et la gestion de l'environnement rural et urbain.

L'A ction comme dispositif d'expérimentation des hypothèses de la recherche: actions de revalorisation de l'environnement dans les terroirs (reboisement, lutte contre l'érosion, aménagement des bas-fonds...); caisses d'associations populaires et villageoises pour financer des micro-réalisations productives; aménagements hydro-agricoles; appui méthodologique à diverses actions menées par les populations; appui financier et technique à des projets.

La Formation à partir de dispositifs de communication horizontale favorisant les auto-apprentissages; formation de paysan(ne)s à des pratiques nouvelles (embouche-fertilité, production de plants...); formation d'animateur(trice)s pour appui à l'auto-formation villageoise; formation des cadres à une approche globale concrète de la gestion des terroirs; formation des responsables de projets à l'évaluation de leurs approches en vue d'améliorer l'appropriation des objectifs par les populations; appui à la formation et à l'auto-formation de forgerons et menuisiers; actions de formation négociées à la demande des partenaires en fonction de l'évaluation des situations; systématisation de la démarche recherche-action-formation par la formation de facilitateur(trice)s populaires; préparations de manuels et de publications pour la diffusion d'outils visant la mise en oeuvre de la recherche-action-formation.

Les objectifs de rapprochement et de facilitation de la coopération entre les peuples et, à l'intérieur de chaque peuple, entre des groupes à sensibilités différentes et diversifiées dans la solidarité complémentaire, que se fixe et concrétise ENDA/GRAF/SAHEL ont rendu aisé l'amour de communauté de perspectives, qui le lie à ECECA et en fait sa "fille naturelle". 


\title{
RÉSEAUX ET ACCÈS À LA DÉCISION: L'EXEMPLE DES GROUPEMENTS FÉMININS AU SÉNÉGAL
}

\author{
Cheikh Badiane
}

\section{La problématique}

Si près de dix ans après la déclaration de la Décennie de la Femme par les Nations Unies, la problématique de la participation des femmes au développement est toujours d'actualité, les termes du débat ont, quant à eux, connu de profondes modifications. L'introduction ces dernières années de l'analyse de genre et la mise en exergue de la question de l'accès des femmes à la décision constituent des données «récentes» qui apportent une tournure nouvelle au débat. Il est apparu en effet que l'intégration de la femme dans le développement, l'un des principaux objectifs de la décennie, ne s'est en définitive matérialisée que par de biens timides actions et une approche stratégique qui n'ont pas été à même de modifier la situation de dépendance des femmes sur les plans économique et social.

Avec le recul, et pour autant qu'on puisse apprécier de manière globale les actions initiées en faveur des femmes, il apparait que la problématique de l'intégration des femmes dans le développement n'est pas une simple question de participation passive à des initiatives conçues ailleurs. L'on admet de plus en plus qu'elle relève davantage du domaine de l'accès à la décision et de l'instauration d'autre formes de rapports sociaux entre les hommes et les femmes. Or, très souvent, ces questions sont liées aux domaines du pouvoir et du changement des mentalités où les réticences sont encore vivaces.

Au Sénégal, c'est à travers l'organisation de leurs groupements en réseaux, dont la mise en place a été facilitée par l'appui de l'Etat, que les femmes rurales tentent d'impulser le changement. Quelle est l'origine de ces groupements et quelles fonctions remplissent-ils? En quoi ces réseaux permettent-ils aux femmes de mieux négocier leur accès à la décision? Les lignes qui suivent, fondées pour l'essentiel sur des données d'une recherche en cours, tentent d'apporter quelques éléments d'appréciation d'une réalité au demeurant fort mouvante et complexe.

\section{Les groupements de promotion féminine}

Née dans le sillage de la Décennie de la Femme, l'organisation des femmes en groupements de promotion féminine est apparue comme une innovation majeure dans les stratégies jusqu'alors poursuivies au Sénégal en matière d'intégration des femmes dans le développement. Faute d'une prise en compte de leurs préoccupations dans les plans nationaux de développement, les autorités ont préconisé l'organisation des femmes en groupements de promotion féminine (GPF) en liaison avec les autorités socio-économiques. L'idée était qu'il serait plus facile pour elles de développer et de gérer des activités communes, de trouver des financements en groupe qu'individuellement. 
Les GPF ont été constitués le plus souvent à partir d'organisations traditionnelles des femmes préexistantes dans les villages. Celles-ci remplissaient diverses fonctions : sociales, culturelles, religieuses, d'entraide financière, matérielle et morale. Mais, contrairement à ces organisations traditionnelles dont la finalité ne semblait pas s'attacher à la réalisation d'activités collectives de développement économique, la spécificité des GPF réside dans le fait que c'est à travers eux que les femmes rurales sont entrées en contact avec les structures d'aide et qu'elles ambitionnent de promouvoir leur développement, particulièrement en milieu rural.

Avec l'émergence des groupements féminins, l'allégement des travaux domestiques est apparue comme une condition préalable à la mise en oeuvre de toute action de développement. Les organismes d'aide l'ont ainsi adopté comme stratégie dans le but de permettre aux femmes de gagner du temps sur les travaux domestiques. Depuis lors, les groupements féminins réalisent en effet une gamme élargie d'activités qui vont de la pratique du crédit à l'alphabétisation, en passant par la mise en place d'infrastructures collectives (boutique communautaire, banque de céréales villageoise, case de santé), l'exploitation de champs collectifs et de jardins maraîchers qui sont autant d'activités qui contribuent, somme toute, à l'amélioration des conditions d'existence des femmes et de leur famille.

Réalisées au coup par coup, et au gré des financements, les actions en direction des femmes n'ont cependant presque jamais fait l'objet d'une planification. La plupart des actions semblaient viser chez la femme non pas l'actrice de développement, mais plutôt la mère/ménagère responsable des activités domestiques.

Or, du fait de leur diversité et de leur imbrication dans l'économique et le social, ces activités réalisées par les femmes se situent dans un champ qui dépasse largement la sphère domestique; en outre, ces dernières années, devant la crise financière et institutionnelle que traverse le pays et face à l'incapacité de l'Etat à satisfaire les demandes sociales, les femmes ont franchi un palier supplémentaire en inscrivant de plus en plus leurs actions dans un domaine qui relève du service public. C'est notamment le cas lorsque, par leurs initiatives, elle suppléent aux carences des pouvoirs publics et des collectivités locales, en mettant en place au niveau communautaire des structures éducatives, sanitaires, sociales, etc. A travers ces actions, elles affirment davantage leur rôle dans le développement et renforcent en même temps leur légitimité. Contrairement à la sphère domestique où leurs actions n'ont pas besoin d'être extériorisées pour leur conférer du pouvoir, dans le contexte des réseaux officiels c'est à partir de la visibilité de leurs actions que les femmes se donnent les moyens de se faire entendre.

Actuellement, il existe plus de 3600 groupements officiellement reconnus, qui mobilisent plus de 400'000 adhérentes sur l'ensemble du pays. Ces groupements ont été au cours de ces dernières années et demeurent à l'heure actuelle les structures qui cristallisent l'essentiel des interventions des organisations gouvernementales et non gouvernementales. Compte tenu de leur importance à l'échelle du pays et de leurs capacités de mobilisation, ils constituent plus que jamais un réel enjeu. C'est sans doute ce qui a motivé les pouvoirs publics à les structurer sous forme d'unions de groupements à travers le pays.

L'objectif officiel est de mieux les appuyer et permettre leur renforcement. D'un point de vue stratégique et politique cependant, le but peut être d'exercer un contrôle sur ce réseau et le cas échéant de l'utiliser, d'autant que les groupements qui le composent sont pour l'essentiel d'origine et d'utilisation gouvernementales. Quelles que soient les intentions des pouvoirs publics, le 
réseau des groupements féminins est un ressort important que les femmes font jouer, contre toute attente, pour négocier leur accès à la décision.

\section{Le réseau des groupements: un enjeu de pouvoir multi-dimensionnel}

En 1987, avec l'appui des services de l'administration, une structure fédérative (la Fédération Nationale des Groupements de Promotion Féminine) a été mise sur pied avec pour objectif de:

permettre aux GPF de se doter d'une instance dirigeante à même de les représenter et de défendre leurs intérêts;

apporter un appui multiforme aux GPF, et jouer si nécessaire un rôle de relais entre les groupements de base et les autres acteurs (Etat, bailleurs de fonds, ONG, etc.).

Cette fédération constitue à ce jour le plus vaste réseau d'organisations de femmes au Sénégal qui met en liaison des femmes rurales à des femmes du milieu urbain. Organisé en unions suivant le découpage administratif du Sénégal, le réseau des groupements féminins a donné naissance à une structure pyramidale qui va des unions locales (situées au niveau des arrondissements) à la fédération nationale, en passant par les départements et régions.

Par exemple, en région de Louga, il existe 593 GPF dont 475 sont affiliés à la fédération nationale. La structuration de ces groupements a permis la mise sur pied à l'échelle de la région de onze unions d'arrondissement, trois unions départementales et une union régionale. Ainsi, la fédération nationale, conçue à partir des unions des différentes régions, est une structure faittière qui remplit diverses fonctions: représentation, négociation, prestation de services. L'instauration de la fédération des groupements féminins a permis dores et déjà de:

rompre l'isolement des groupements féminins;

élargir leur horizon et leur rayon d'action;

accroitre dans une certaine mesure leurs moyens financiers et/ou leurs possibilités d'accès aux moyens financiers et matériels.

C'est à travers la fédération que les femmes rurales et leurs leaders négocient désormais leur accès aux décisions, en particulier dans les domaines politique, économique et social, avec des résultats variables.

Sur le plan politique

Il existe à ce niveau une sorte de contradiction entre le rôle actif et décisif que jouent les femmes sur l'échiquier politique (au Sénégal les femmes ont toujours joué un rôle déterminant dans la désignation des responsables politiques) et la faiblesse de leur influence sur les décisions politiques, une fois le pouvoir en place. Cette faiblesse apparait d'une part dans leur représentation 
minoritaire au sein des instances de prise de décision, ainsi que les statistiques en annexe le font apparaitre; elle se manifeste d'autre part dans l'insuffisante prise en compte de leurs rôles dans le développement (accès aux ressources et aux moyens de production, contraintes, etc.).

Ainsi l'accès à la décision, notamment sur le plan politique, est assez timide au regard du rôle et de l'importance des femmes sur la scène politique. Au niveau rural, le non-accès des femmes au conseil rural est une illustration éloquente de ce déphasage, quand on sait à quel point leur contribution est importante dans le développement rural.

Cependant, c'est à travers le réseau des groupements féminins que les femmes tentent actuellement de faire introduire le système de quota dans les instances de décision, de même qu'elles exercent une pression pour une révision des modes électoraux au niveau des conseils ruraux de manière à faire émerger les femmes.

Il faut noter également que les représentantes des GPF participent aux instances des conseils locaux, départementaux et régionaux de développement (CLD, CDD et CRD). Plus que l'efficacité de cette participation qui n'a pour l'instant aucune influence sur les grandes décisions, il convient de considérer ce principe comme une avancée née de la revendication des femmes et aussi comme une formidable opportunité d'apprentissage et de formation qui sont de étapes nécessaires vers la maitrise des voies d'accès à la décision.

\section{Sur le plan économique}

L'absence d'une politique volontariste de prise en compte des préoccupations des femmes en matière de développement a pour conséquence une difficulté pour celles-ci d'accéder aux ressources, à la technologie, à la formation et au crédit, qui passent notamment par le canal des pouvoirs publics. Aussi la FNGPF devenue ONG permet-elle d'élargir le champ d'action des femmes qui cherchent désormais à se connecter sur le réseau international en sollicitant notamment le financement d'organismes comme la Banque mondiale et le FIDA par exemple.

Sur le plan national, la seule mobilisation des droits d'adhésion des groupements de base à la fédération a permis de disposer de moyens suffisamment importants pour déposer un fonds de garantie à la banque (la Caisse Nationale de Crédit Agricole du Sénégal), et mettre en place au niveau de chaque région un fonds géré directement par les responsables locaux des GPF. Ce fonds vise essentiellement à autofinancer des micro-réalisations.

Au-delà des aspects financiers, la gestion décentralisée du fonds donne confiance et légitimité aux leaders locaux et contribue à l'émergence ou au renforcement d'un espace social orienté vers la solidarité, les alliances et les innovations.

Quant à l'impact sur les femmes membres à proprement parler, il peut s'illustrer à travers les possibilités d'accès au crédit offertes aux femmes rurales grâce au réseau des groupements féminins. En effet, l'organisation collective des femmes rurales est un moyen d'obtenir des crédits auprès des institutions de développement. Tandis que la femme en tant que productrice individuelle n'est pas encore reconnue comme bénéficiaire potentielle en raison de son manque de garantie, les groupements sont des partenaires reconnus. Aussi, c'est grâce à eux que les femmes parvien- 
nent à obtenir des moyens auprès des $\mathrm{ONG}$ ou des projets qui interviennent en milieu rural. Tout cela fait partie des potentialités économiques des groupements de femmes.

En outre, les changements sur le plan économique en entraînent d'autres sur le plan social, qui concernent, d'une part, les femmes elles-mêmes, et d'autre part, les rapports sociaux entre les hommes et les femmes au sein des villages.

\section{Sur le plan social}

En tant que lieu d'apprentissage, de formation et de conscientisation, le réseau des GPF est avant tout un cadre d'affirmation de la personnalité. En effet, à travers lui, les femmes augmentent leurs possibilités de communiquer ensemble et d'échanger des idées, de trouver des solutions, de réaliser des projets, etc. Par ailleurs, le degré d'ouverture sur l'extérieur semble plus élevé chez les femmes membres des groupements féminins affiliés au réseau que chez les non-membres. C'est que ces groupement offrent de nombreuses possibilités de contacts et de collaboration, en particulier avec des acteurs sociaux et institutionnels extérieurs au village, ce qui fait avancer les savoirs et savoir-faire des femmes et renforce leur confiance en elles-mêmes. Grâce à la médiation de la fédération, des opportunités naguère inexistantes s'offrent maintenant. Tandis qu'il y a quelques années encore, aller au marché hebdomadaire du village voisin pour y vendre quelques produits constituait pour nombre de femmes rurales une des rares possibilités de quitter le village et d'entrer en contact avec d'autres femmes (et ceci juste le temps d'un échange marchand), l'avènement des GPF a bousculé les traditions et ouvert les femmes sur l'extérieur. De nouvelles attitudes et pratiques, difficiles à réaliser naguère, sont dorénavant courantes: absence des femmes du village pendant quelques jours pour assister à un séminaire (portant de surcroit sur la planification familiale par exemple), gestion par les femmes d'activités communautaires génératrices de revenus, prise de parole publique dans des assemblées mixtes, etc. sont autant de choses rendues possibles par les GPF qui disposent de plus en plus de légitimité et agissent comme force de négociation au profit des femmes.

Lors de la dernière conférence africaine sur les femmes (novembre 1994), une dizaine de représentations des groupements féminins de la région de Louga se sont rendues à Dakar. Le fait d'avoir contribué pour l'équivalent de 20 dollars chacune afin de pouvoir participer au forum des ONG n'est pas sans intérêt. Il traduit la prise de conscience des femmes et leur disposition à payer au besoin certains services qu'elles considèrent comme utiles pour leur accès au processus de décision.

\section{Conclusion}

Les réseaux des groupement féminins sont donc à l'origine d'une nouvelle dynamique sociale. Ils contribuent aux changements dans les rapports sociaux qui s'opèrent progressivement au sein des villages. Ceux-ci sont certes plus difficiles à percevoir que les changements économiques qui sont davantage de l'ordre du mesurable. Les changements sociaux transparaissent dans les discours et les attitudes. Cependant ces changements dans les rapports entre les hommes et les femmes ont été rendus possibles, dans la plupart des cas, par les performances économiques des femmes et leur appartenance à un réseau de solidarité qui leur confère plus de poids. 


\section{Documents de référence:}

DIOUF M., SOW F., Femmes sénégalaises à l'horizon 2015, Population Council, Ministère de la Femme, de l'Enfant et de la Famille, Dakar, 1993.

MIGNOT LEFEBVRE Y., «Les femmes dans l'économie, de l'invincibilité à de nouveaux modes d'organisation», Revue Tiers-Monde, t. XXVI, n¹02, avril-juin 1985.

Ministère du Développement social, Etude sur l'intégration de la femme dans les plans et programmes de Développement: cas du Sénégal, Dakar, 1988.

Ministère de la Femme, de l'Enfant et de la Famille, Rapport national sur les femme. Lutte pour l'égalité, le développement et la paix, Dakar, avril 1994.

REVEYRAND O., «Les associations féminines en Afrique noire: l'exemple de la Casamance», Le mois en Afrique, Etudes politiques, économiques et sociologiques africaines, 1987.

SAVANE M.A., Les projets pour les femmes en milieu rural au Sénégal, Genève, BIT, 1983.

SOW F., Enquête sociologique sur les groupements féminins autour de Nadadji-Civol (Département de Matam-Sénégal), janvier 1990. 


\begin{abstract}
ANNEXE
Quelques statistiques sur les femmes au Sénégal

\section{Nombre de femmes élues aux conseils municipaux}

$1984: 117$

1990 : 354; soit un triplement du nombre de femmes sur une législature. Mais en valeur relative, on constate que le nombre de femme ne représente en 1990 qu'environ 15\% du nombre total des conseillers municipaux dans les 40 communes du Sénégal.
\end{abstract}

\title{
Nombre de femmes élues aux conseils ruraux
}

$1984: 155$

1990 : 425; ce chiffre ne représente que 5,6\% de l'effectif total des conseillers ruraux dans les 317 communautés rurales du Sénégal.

Ainsi, le pourcentage des femmes au sein des conseils municipaux et ruraux a marqué un certain progrès, passant en 6 ans de 272 à 779. Mais en réalité, la représentation des femmes à l'issue des dernières élections de 1990 demeure tout à fait dérisoire: 7,92\% sur l'ensemble des élus du Sénégal.

\section{Par ailleurs, le Sénégal compte:}

nombre de ministres femmes: 2 sur 29

nombre de femmes au Parlement (7e législature: 7 femmes pour 113 hommes)

nombre de femmes au Parlement (8e législature: 11 femmes pour 109 hommes)

une seule femme maire

Corps des magistrats: 27 femmes sur 218

Ordre des avocats: 10 femmes sur 256 avocats (3,9\%)

Source: Ministère de la Femme, de l'Enfant et de la Famille, Rapport national sur les femmes. Lutte pour l'égalité, le développement et la paix, Dakar, avril 1994. 


\title{
L'EDUCATION SOCIO-FAMILIALE: LA PLACE DU COUPLE
}

\author{
PAPE DIOUF
}

\section{RAPPEL MÉTHODOLOGIQUE}

Il est convenu, de nos jours, de retenir la catégorie "femmes" en la rattachant souvent au concept "développement". Mais il reste entendu que, du point de vue sociologique, on ne peut pas se limiter à cette catégorie trop vaste, molle et peu analytique. En particulier, en milieu urbain, il est important de garder à l'esprit la grande disparité parmi cette catégorie des femmes. Par exemple à Dakar, Harare, Abidjan, Nairobi ou dans d'autres grandes villes des pays en développement, il existe généralement une large différence dans le comportement des femmes mais aussi dans leurs rapports avec les hommes, suivant des critères comme l'âge, le niveau d'instruction, le type d'activité professionnelle, la religion, etc. Toutefois, nous utiliserons cette catégorie par commodité. De même utiliserons-nous la notion de ville, distinctive du monde rural, sans ignorer qu'il s'agit là de notions dont l'analyse montre la complexité des interrelations et flux de tous ordres, y compris sur le plan environnemental mais également culturel.

Le séminaire ayant retenu ces catégories, nous allons les employer dans cette modeste contribution à la réflexion.

\section{DE LA PERTINENCE DU COUPLE FEMMES-ENVIRONNEMENT}

L'observation et l'analyse des activités des femmes dans toutes les sociétés rurales débouchent clairement sur les interactions permanentes entre les femmes et les ressources naturelles et l'environnemment, cela de manière générale. Elles jouent un rôle de premier plan dans la gestion de l'eau et de la forêt et sont chargées de la salubrité de l'environnement immédiat. Même si cette fonction n'est pas valorisée dans tous les groupes sociaux, elle est reconnue et procède même de la division sexuelle du travail. Les relations de ces femmes avec leur environnement dépassent largement la dimension utilitaire et relèvent des domaines de l'affectif et du symbolique. Ce rapport à l'environnement est transmis par l'éducation socio-familiale traditionnelle, intégrant à la fois les savoirs et les comportements nécessaires à la bonne gestion de l'environnement dont les femmes sont en fin de compte les gardiennes et les protectrices. C'est ainsi qu'elles définissent par des règles précises l'utilisation des ressources entre elles, y compris des sanctions sévères aux contrevenantes. Par exemple on retrouve les mêmes formes de sanctions dans des contrées aussi éloignées que le Sénégal et le Tchad, qui consistent pour le groupe de femmes à mettre en quarantaine toute femme ne respectant pas les règles établies pour la gestion collective d'un puits. Une telle punition est un des pires sévices qui puissent exister et décourage valablement toute velléité de ne pas observer le règlement établi. 
Les espaces humains en question sont d'échelle réduite, facilitant le contrôle social, avec des rapports sociaux plus disciplinés que dans des établissements humains de plus grande envergure. La responsabilité des femmes dans le domaine de l'environnement est claire et acceptée par le reste de la société, dans la plupart des sociétés rurales africaines. Quel que soit le système de propriété foncière, l'accès et le contrôle des ressources de l'environnement sont assurés par les femmes qui jouent un rôle essentiel dans l'éducation à l'environnement aussi bien des filles que des garçons, voire des adultes. Selon les classes d'âge et le sexe, il s'agit de connaissances précises et de comportements véhiculés, notamment par les femmes, au plan familial comme sociétal. C'est ainsi que l'on retrouve les dimensions environnementales physiques et spirituelles dans la plupart des rites de passages chez les garçons comme chez les filles.

\section{L'URBANISATION COMME REMISE EN CAUSE DU PARADIGME}

Les processus d'urbanisation, principalement en Afrique, ont complètement bouleversé le paradigme des relations entre femmes et environnement analysé ci-dessus. Les transformations se sont opérées suivant deux phénomènes essentiels dans le développement des villes, à savoir:

- la massification qui accompagne l'urbanisation,

- la scolarisation.

Avec la massification, le couple femmes-environnement n'a pas pu résister. L'augmentation drastique des besoins dans la cité (contrairement au village) a provoqué un accroissement trop rapide de l'exploitation des ressources naturelles, et donc une augmentation des besoins de salubrité. Avec une telle évolution, les limites des femmes à assurer leur rôle traditionnel ont été vite atteintes. Les bouleversements importants nés de cette évolution ont conduit à des mutations profondes des modes de production et de consommation dans les villes. Les relations avec l'environnement en particulier connaissent des changements profonds.

La scolarisation, devenue mode d'éducation et de formation dominant, n'intègre que très peu les dimensions environne-mentales, ni pour les filles, ni pour les garçons.

Dans ce contexte les femmes ont perdu à la fois l'accès et la reconnaissance de leur rôle sur les ressources naturelles. De nouveaux acteurs se sont positionnés pour l'exploitation tous azimuts de ces ressources que l'urbanisation-massification a transformée en biens d'échange, contrairement à leur statut de biens d'usage qu'elles ont en milieu rural. Dès lors la gestion et le contrôle des ressources de l'environnement sont devenus un enjeu de taille dont les conséquences ne se limitent pas seulement aux villes. L'exemple de la forêt est typique quant on sait la pression et la dégradation dont elle fait souvent l'objet de la part d'exploitants, généralement installés en ville et dont les revenus profitent presque exclusivement au milieu urbain au détriment du monde rural environnant, et de la société dans son ensemble. Les femmes rurales paient un lourd tribut à ce sujet, car elles doivent souvent parcourir de longues distances pour chercher le bois de chauffe devenu très rare dans certains pays comme en ceux de la zone sahélienne.

Dans le modèle rural, c'est au moins la moitié de la société qui est responsable des problèmes d'environnement, en l'occurrence les femmes. En ville, cette responsabilité s'estompe et la régulation entre la société et l'environnemnt, au sens large, n'est pratiquement plus assurée. D'autres types de rapports sociaux se sont tissés dans le cadre de l'exploitation et de la protection de l'en- 
vironnement. La structure de ces nouveaux rapports n'intègre pas le rôle traditionnel des femmes dans les sociétés rurales, et tend à renforcer l'intervention de l'Etat. Comme c'est souvent le cas dans les pays en développement, l'Etat est mal préparé à cette fonction et les politiques et stratégies qu'il met en place sont peu efficaces.

L'urbanisation dans les pays en développement rime souvent avec insalubrité, désertification et problème d'eau, autant de domaines où les femmes sont directement concernées, mais ne sont plus responsabilisées, ni par le biais de la scolarisation, ni par les stuctures sociales en place.

\section{DEUX PISTES D'ANALYSE SE DÉGAGENT}

Pour récapituler sur cette brève intervention tendant à susciter la réflexion quant aux effets de l'urbanisation sur le couple femmes-environnement, nous allons dégager deux pistes qui nous paraissent intéressantes à continuer d'explorer :

- Le couple femmes-environnement a fonctionné et a fait ses preuves en milieu rural grâce notamment à l'éducation socio-familiale traditionnelle permettant une responsabilité particulière des femmes dans le domaine de l'environnement. Il est essentiellement fondé sur une logique non marchande et les rapports entre société et environnement sont régis par des valeurs d'usage, favorables à l'équilibre de ces relations.

- L'urbanisation accélérée dans les pays en développement a provoqué une massification qui a conduit à une dérespon-sabilisation des femmes sur les questions d'environnement et une sorte de démission de la société, fléaux contre lesquels la scolarisation apporte peu de solutions. Ni l'Etat, ni la société n'ont pu donner de réponse efficace à cette situation qui a complètement bouleversé les rapports à l'environnement; ceux-ci sont fondés en ville surtout sur une logique marchande marquée par des valeurs d'échanges provoquant des déséquilibres inquiétants qui ne se limitent pas aux frontières de la ville mais affectent l'ensemble des pays concernés.

Enfin la réflexion menée ici ne se réclame ni du dualisme théorique ville-campagne, ni d'un pas séisme forcené. Elle s'inspire de quelques années d'expérience vécue dans de nombreux pays d'Afrique où se pose avec acuité la question de la gestion de l'environnement. Face à ce constat, il nous paraît opportun, dans la recherche de solutions, de partir des réalités en place et de s'interroger systématiquement sur le rôle que les femmes pourraient certainement jouer dans les politiques et les stratégies de gestion de l'environnement. Il ne s'agit pas, dans notre entendement, de retourner au passé et au modèle rural, mais d'associer étroitement les femmes dans la recherche d'alternatives adaptées au contexte actuel, et de fixer avec elles les nouveaux rôles qu'elles pourraient jouer, aussi bien dans la définition que la mise en oeuvre des politiques et des stratégies environnementales. A l'heure où les Plans d'Action pour l'Environnement (PAE) constituent un instrument privilégié des politiques des pays africains, il serait intéressant de voir la place réservée aux femmes - des villes comme des milieux ruraux - dans l'élaboration de ces plans. Espérons qu'une meilleure prise de conscience s'opère au niveau des autorités, particulièrement en Afrique. 


\title{
MENUISIERES, CHARPENTIERES, MAÇONS... LA COOPERATIVE DE MULUKUKU AU NICARAGUA ${ }^{1}$
}

\author{
GRETHEL SEQUEIRA MONTOYA
}

\begin{abstract}
"Nous avons décidé de travailler ensemble. Nous rêvions d'avoir une maison à nous, pour être indépendantes des hommes et décider ce que nous voulions faire; nous ne voulions plus être maltraitées. Nous ne supportions plus la violence. Autour de nous c'était la guerre. A cette époque de la révolution sandiniste, on parlait beaucoup de coopératives, de collectifs, de solidarité, de faire les choses ensemble. C'était un projet révolutionnaire. Ce fut notre projet.
\end{abstract}

Après le passage de l'ouragan tout était détruit. Nous n'avions plus rien: plus de maisons, plus de revenus, aucun appui économique. Beaucoup de femmes étaient chefs de famille. Il nous fallait une réponse urgente aux premières nécessités, celles de reconstruire des maisons pour nous et pour les autres membres de la communauté. Il nous fallait prouver aux hommes qui nous étaient hostiles que nous pouvions nous organiser. Ils se moquaient de nous, disaient que nous n'y arriverions pas, que les briques de ciment que nous faisions étaient inutilisables. Certains interdisaient à leur femme de participer, d'autres nous traitaient de folles, de sales, de "mangeuses de ciment" plutôt que d'être de bonnes ménagères... Nous avons dû apprendre à faire des choses auxquelles nous n'avions jamais pensé: de la menuiserie, des briques, des portes et des fenêtres, manier de lourdes machines électriques, utiliser des clous et des vis, conduire des camions, faire des maisons solides qui ne s'écrasent pas au premier coup de vent. Et puis nous avions des enfants, beaucoup d'enfants qui manquaient de soins, qui n'allaient pas à l'école. Nous devions aussi nous défendre des mauvais traitements des hommes, de l'abandon des pères de famille, de la guerre..." Ainsi parlent les femmes de la coopérative de Mulukuku.

1. Ce texte a été élaboré à partir

- des interventions de Grethel Sequeira Montoya au Colloque "Femmes, villes, environnement" (objet de la présente publication),

- des interviews avec Olivia Heussler, photographe, Suzanne Lerch, architecte, Yvonne Preiswerk, anthropologue,

- de l'article de Catherine VUFFRAY et Olivia HEUSSLER dans Entwicklung Développement n 46/1995 de la Direction de la coopération au développement et de l'aide humanitaire (DDA) Suisse et - des archives et rapports de l'Oeuvre suisse d'entraide ouvrière (OSEO), partenaire du projet. 


\section{MulukuKu, UNE VILLE QUI VA DE L'AVANT}

Mulukuku se situe sur l'une des deux grandes routes nicaraguayennes qui mènent à la côte atlantique, sur la frontière entre la région de Matagalpa et celle d'Atlantico Norte. Le pont sur le Rio Tuma à Mulukuku - le seul qui supporte le passage de véhicules - joue un rôle essentiel dans le développement de la localité. Pendant la guerre civile qui débuta en 1983 contre la contra, Mulukuku a souvent été le théâtre d'affrontements armés. C'était alors un petit village d'une vingtaine de familles. En 1985 Mulukuku est devenu une des quinze implantations que le Gouvernement avait décidé d'établir pour les déplacés de la guerre. Nombre d'habitants, surtout des femmes avec leurs enfants, qui avaient jusqu'alors vécu dispersés dans la région sont ensuite venu chercher refuge à Mulukuku. C'est avec des moyens extrêmement modestes que ces femmes ont tenté de se forger une nouvelle existence. En raison de la proximité d'une caserne et du nombre de chauffeurs faisant halte à Mulukuku sur la difficile route vers la côte atlantique, la prostitution a été florissante. Pour nombre de femmes, elle représentait le seul moyen de survivre. Le maire de l'époque et sa femme ont été très préoccupés par cette évolution et ont commencé à organiser les femmes. Parmi les premiers projets productifs, on note la construction d'un moulin à maïs et d'une boulangerie.

En 1988, les inondations provoquées par l'ouragan "Joan" ont détruit une grande partie de Mulukuku, ainsi que le pont sur le Rio Tuma; 207 familles (environ 1500 personnes) ont tout perdu. Des habitants motivés ont alors fondé le "Comité de Reconstruccion" et ont émis l'idée de reconstruire Mulukuku sur l'autre rive du fleuve, sur un emplacement plus élevé et plus sec. Les organisations féminines ont joué un rôle actif dans ce processus. Soutenues par des organisations nationales et internationales ${ }^{2}$, les femmes ont construit une briqueterie et une menuiserie, afin de produire elles-mêmes le matériel nécessaire à la construction de leurs maisons. De plus, une école et un centre de santé pour femmes ont été ouverts. Le nouveau pont a pu être inauguré en 1991 et, depuis lors, le développement de Mulukuku va bon train. Le nouvel emplacement, plus élevé, accueille toujours plus de nouveaux habitants. On voit fleurir partout des petits magasins et des entreprises.

Pourtant, malgré toutes ces activités, les revenus de la plupart des habitants leur suffisent à peine pour survivre. Comme par le passé, des affrontements armés secouent cette région périphérique. Le taux de criminalité est élevé et les catastrophes naturelles, tel l'ouragan "Gert" qui a provoqué d'importants dégâts en 1993, rendent encore plus difficiles les conditions de vie de la population.

\section{LA COOPÉRATIVE DE FEMMES "MARIA LuISA ORTIZ"}

Dans le sillage du "Comité de Reconstruccion" naît le collectif "Maria Luisa Ortiz" fondé alors par une quarantaine de femmes. Nous avons organisé quatre brigades de dix femmes pour construire dix maisons. Dans chaque brigade, une femme s'occupait des enfants, à tour de rôle. Nous avons appris à faire des briques avec du sable, de la terre et un peu de ciment. Un formateur nous a montré comment utiliser une machine, très lourde à manipuler que nous avions

2. ONG nationales: Institut Jeau XXIII, Projet Cristo Rey. ONG internationales: Agriculteurs pour la paix du Canada, Habitat pour l'Humanité, groupes de solidarité des USA et surtout l'OSEO, Oeuvre suisse d'entraide ouvrière, Zurich. 
trouvée grâce à une organisation jésuite. Un organisme canadien a mis à disposition des maçons pour diriger le travail. Les femmes qui comprenaient plus vite aidaient les autres. Nous avons vendu des briques aux autres organismes qui construisaient des maisons. Nous pouvions faire de 70 à 100 briques par jour. Comme il fallait construire des toits, des portes, des fenêtres et des meubles, il a fallu créer une menuiserie gérée elle aussi par les femmes. Ainsi sont nées les menuisières et la menuiserie.

Au début les travaux du bois nous ont semblé particulièrement difficiles. Nous n'avions que des outils artisanaux courants pour scier, façonner et travailler le bois. Le travail à la menuiserie a été bien plus laborieux que la fabrication assez simple des briques. En effet, sans connaissances particulières des métiers du bois, nous avons non seulement dû inventer nos propres techniques mais encore nous adapter aux difficultés quisurgissaient de jour en jour. Lorsque nous avons reçu des machines, nous avons tenté de passer du travail proprement manuel à un outillage qui nous semblait sophistiqué et difficile. Peu à peu il a fallu amadouer ces techniques mécaniques et industrielles nouvelles. Ce fut là pour nous une révolution dans notre art de travailler le bois et de produire les éléments essentiels à la construction des maisons. Nous nous réjouissons de dire que ce fut une expérience difficile mais hors du commun. Et, comme pour les briques, notre travail à la menuiserie nous a permis de vendre nos produits à Mulukuku de même qu'à Matagalpa et de nous introduire ainsi sur le marché des articles du bois et des briques.

La briqueterie et la menuiserie offrent des postes de travail à une vingtaine de femmes et génèrent de petits profits qui servent à financer d'autres projets productifs.

En 1989, le terrain de ce qui n'était pas encore un véritable village a été vendu, par l'intermédiaire de la mairie, au gouvernement régional de Matagalpa. Les hommes et les femmes de la coopérative de Mulukuku ont ensuite obtenu leur titre de propriété. Mais comme nous, les femmes, voulions être les propriétaires des maisons, nous ne voulions pas que les hommes, en nous aidant et en y investissant du travail, disent par la suite que la maison leur revenait. C'était difficile, c'était une lutte. Un temps, il était interdit aux hommes d'entrer à l'atelier, parce qu'ils étaient agressifs ou moqueurs. A présent, ils viennent quelquefois nous aider; mais on ne les accepte toujours pas quand il s'agit de prendre des décisions.

Le travail est planifié et partagé. Maintenant, cent femmes au total ont leur maison à elles. Elles vivent avec leur compagnon ou bien seules. Cela n'étonne plus personne, dans la communauté, que les femmes soient propriétaires de leur maison; c'est maintenant un fait accepté.

\section{Une école et une clinique}

La clinique est importante parce que c'est la seule de la région; mais elle n'a pas de médecin. Elle est aussi ouverte aux hommes. On s'y occupe surtout de contrôles gynécologiques et de grossesse, de planning familial, de médecine naturelle, de nutrition, etc. Il y a aussi un service d'ophtalmologie et de dentiste. Les consultations sont payantes pour ceux qui le peuvent (5 Cordobas pour un adulte, l Cordoba pour un enfant). A la clinique seulement, le travail des femmes est volontaire car il s'agit d'un travail social.

Et puis, il faut parler de la stérilisation, car c'est une nécessité chez nous: certaines femmes ont 10, voire 13 enfants jeunes; l'an dernier, une femme de 39 ans a mis son vingtième enfant au 
monde. Avant, nous allions en camionnette à Managua; avec le déplacement, cela prenait une semaine en tout. Depuis mars 1994, une unité mobile vient deux à trois fois par an à Mulukuku; les femmes qui viennent de la montagne pour l'opération peuvent ensuite se reposer à la clinique. Jusqu'à présent, 170 femmes environ se sont fait stériliser, mais seulement quatre hommes.

\section{ET APRÈS}

Une fois le plus gros des travaux de construction terminés, la coopération s'est concentrée dès 1991 sur la formation et le perfectionnement des menuisières et le travail communautaire avec les femmes. Après s'être consacrées avec beaucoup d'engagement aux activités productives (menuiserie, transports avec le camion de la coopérative, fabrication de briques et moulin à maïs), elles ont mis sur pied un comité de parents et ont obtenu que le gouvernement envoie des enseignantes à Mulukuku. Cependant, le nombre des enfants s'étant accru, l'école a eu besoin d'occuper toutes ses salles. C'est pourquoi les femmes ont transformé une maison qui sert depuis 1993 de centre pour les femmes et offre suffisamment de place pour abriter le dispensaire et les autres activités.

Depuis 1991, la coopérative de femmes organise aussi régulièrement des cours de formation et des rencontres pour débattre des droits des femmes, des mauvais traitements, de la discrimination, de la nutrition et du planning familial. Elle met en outre sur pied des cours de formation systématiques en matière d'administration, d'organisation, de planification du travail et d'alphabétisation; un grand nombre d'entre elles sont semi-alphabétisées. Plus de 1000 femmes ont ainsi pris part aux activités.

Alors que la population, surtout les hommes, a fait, au départ, preuve de beaucoup de scepticisme, voire d'hostilité face à l'initiative des femmes, la coopérative est peu à peu parvenue à se faire accepter et même apprécier par la commune. Les femmes jouent aujourd'hui un rôle important dans l'organisation de l'entraide et dans la création de nouvelles sources de revenus. En organisant des rencontres sur la discrimination et la violence envers les femmes, elles ont lancé un processus de prise de conscience, également auprès de la population masculine, qui sert à promouvoir des valeurs telles que l'égalité des chances et le respect mutuel.

Si l'on devait aujourd'hui énumérer les objectifs de la coopérative des femmes, ils seraient les suivants :

- promouvoir l'égalité entre hommes et femmes et sensibiliser la population à la nécessité de faire participer les femmes à tous les aspects de la vie sociale et politique;

- encourager l'autodétermination et la collaboration parmi les femmes et renforcer la notion de solidarité entre elles;

- renforcer l'autogestion, accroître les connaissances administratives et professionnelles, dans le but de créer de nouvelles sources de revenus pour les femmes;

- poursuivre la formation continue des menuisières (stylisme, entretien des machines, diversification de la production, comptabilité et administration); dès 1995, les coûts de cette formation seront financés par les revenus de la menuiserie;

- organiser encore des cours d'alphabétisation pour les femmes, des cours sur les médecines naturelles et sur la nutrition, ainsi que le perfectionnement des agentes de la santé ; 
- mettre sur pied des manifestations et des rencontres destinées aux femmes, aux hommes et aux jeunes, pour débattre de thèmes tels que les relations entre hommes et femmes, la discrimination et la violence, la prévention pour les jeunes (loisirs, toxicomanie), la prise de conscience des problèmes liés à chaque sexe, les questions liées à la santé et à l'environnement;

- apprendre à évaluer le travail effectué mais aussi les méthodes d'enseignement et le matériel didactique.

\section{COMMENT SE VIT CETTE EXPÉRIENCE EXTRAORDINAIRE?}

Il n'a pas été simple de négocier nos projets avec nos partenaires nationaux et étrangers qui, au début, ne comprenaient pas toujours nos propres idées, nos critères politiques. Personne ici n'est universitaire et on nous demandait des informations, des rapports, des évaluations que nous ne pouvions pas donner. Il fallait plus de confiance et nous souhaitions des interlocuteurs plus flexibles qui aillent sur le terrain au risque de se mouiller et de se salir. Nous ne voulions pas non plus imposer les politiques de ces organisations à nos populations alors qu'elles ne prenaient pas la peine de nous connaitre, nous, nos rythmes, nos nombreuses activités paysannes, domestiques, familiales. Souvent c'était leurs projets, pas les nôtres.

Nous avions besoin d'être valorisées, de pouvoir nous fixer des objectifs sans délais fixés. Pourtant il nous fallait de l'aide technique, de l'organisation. Peu à peu, on nous a pris au sérieux. On s'est rendu compte que nous oeuvrions au développement de la communauté et des familles de Mulukuku. Il faut souligner que c'est probablement grâce à une femme d'une ONG suisse que le courant a passé plus aisément. Elle a compris notre manière de travailler, elle nous a fait confiance, nos projets de solidarité entre femmes ont séduit. Il fallait absolument intégrer à nos projets, qui sont nombreux, la réflexion sur la violence entre hommes et femmes.

\section{QUESTIONS DE GENRE}

La charge domestique souffre de nos activités mais il est vrai que nous, qui travaillons à construire des maisons et qui oeuvrons dans les ateliers, avons davantage à faire. Nous démultiplions nos énergies mais nous nous organisons aussi. Nous insistons beaucoup sur le processus d'éducation avec les enfants. Dans le futur il y aura aussi davantage d'hommes qui participeront au travail domestique. Il faut dire avec le recul que nous ne sommes pas affligées et prises de mauvaise conscience lorsque les repas ne sont pas prêts à temps et que les hommes ne sont pas immédiatement satisfaits. Avant, nous avions beaucoup de remords :

"Mon Dieu, le repas n'est pas prêt, les pauvres... les repas sont froids, le linge n'a pas été lavé à temps...

Maintenant on s'en fiche. S'ils ne sont pas contents, personne ne les empêche de laver, de faire à manger..."

Cette tranquillité qui nous est venue en travaillant ensemble, c'est l'une des choses les plus importantes qui nous soit arrivée.

On dit souvent qu'en Amérique latine la lutte des femmes est plutôt bourgeoise et élégante mais ici ce sont des femmes paysannes qui se sont appropriées ces pouvoirs par nécessité et par lutte. La situation était très pratique. Dans les zones de guer re, les femmes, en plus d'avoir la responsabilité domestique parce que les hommes allaient se battre, ont investi l'espace laissé par les hommes. Nous avons été obligées de faire face aux situations de ce type d'urgence. En leur 
absence, nous devions tout affronter et quand ils revenaient, ils nous traitaient comme des fillettes qui ne pouvaient pas gérer les choses.

Par ailleurs la violence est un des facteurs marquants dans nos décisions. C'est vrai que nous sommes pratiquement parties de rien. Nous voulions une maison à nous pour nous permettre de décider si nous voulions vivre avec la violence ou si nous avions la possibilité de négocier pour subir moins de violence. Au fur et à mesure de la mise sur pied de notre coopérative, nous nous sommes rendu compte qu'il y avait d'autres groupes de femmes en Matagalpa, dont un qui travaillait sur les rapports entre femmes et hommes. Ainsi nous avons commencé à réfléchir sur ces rapports de pouvoir dans notre quotidien. Qu'allons-nous faire, nous les femmes? Quelles sont nos charges? Pourquoi devons-nous être aussi maltraitées? Y a-t-il une raison de l'accepter? Cela nous paraissait découler immédiatement de notre réflexion sur les rapports de pouvoir.

Au début nous avions peur de parler de thèmes aussi ardus que les relations sexuelles, nous n'en avions pas le courage. Cela nous paraissait une question très difficile. Maintenant nous avons plus de sécurité et nous pouvons parler avec respect, avec solidarité de ces problèmes qui nous terrifiaient. Personnellement, j'ai trouvé que c'était difficile, mais nous devons continuer à porter notre réflexion sur ces libertés aussi. La pratique sur le terrain nous a donné une force incroyable pour affronter ces problèmes.

Les années de la révolution sont dans le coeur de chaque Nicaraguayen ainsi que tous les concepts de collectivité, de propriété, de coopérative, d'égalité. Si nous les femmes parlons de propriété, d'égalité au niveau national, d'injustice, d'opportunités pour les paysans d'avoir accès à la terre, de financement pour l'éducation des paysans, pour qu'ils apprennent à lire, pourquoi ne pas le faire au nom des femmes? Ainsi nous avons sollicité l'aide des autorités de la communauté de Mulukuku qui nous ont approuvées. Certains nous ont poussées à continuer. Nous nous sommes appropriées des concepts de la révolution pour obtenir des choses qui nous semblaient simples et fondamentales pour les femmes. C'était un acte important pour nous autres.

Mulukuku a été détruite par l'ouragan. La première nécessité c'était de reconstruire les maisons. Nous nous sommes organisées. Nous devions travailler ensemble. C'est ainsi que nous avons grandi petit à petit.

Actuellement nous pensons que Mulukuku a été un exemple car, après la transformation due au changement de pouvoir, on peut constater une dégradation générale. On nous a enlevé la banque, le médecin, les services agraires. Il ne reste que la Municipalité pour prélever des impôts sur les quelques têtes de bétail qui nous restent. Nous avions pourtant besoin de services de santé, de davantage de maisons; cette année, nous avons terminé une quarantaine de nouvelles maisons pour des femmes et nous commençons une dizaine d'habitations supplémentaires. L'important n'est pas que les maisons servent seulement aux femmes. Mais nous étions prêtes pour les demandes administratives, nous savions comment gérer; nous avons appris comment prendre en charge les situations.

Cela nous ramène aux relations hommes/femmes. Il ne s'agit pas seulement que nous nous affirmions en tant que groupes de femmes pour le développement mais que nous oeuvrions aussi pour que la mentalité des hommes change envers nous. Nous jouissons de beaucoup de solidarité entre femmes, mais il importe que les hommes et surtout les jeunes hommes apprennent à négocier avec nous autant le travail que les relations de pouvoir et les décisions. C'est le début d'une prise de conscience de l'égalité des droits et des chances entre hommes et femmes. 


\title{
LES FEMMES ET UN MOUVEMENT POPULAIRE D'HABITATION: SÃO PAULO, BRESIL
}

\author{
JEANNE BISILLIAT
}

L'existence des mouvements populaires qui constituent, pour la partie la plus défavorisée de la société civile, et donc pour les femmes, un réel apprentissage à la citoyenneté doit retenir notre attention.

Ces mouvements que l'on rencontre presque exclusivement en Amérique latine ont été étudiés, jusqu'à ces dernières années, comme les autres phénomènes sociaux sous l'angle trompeur d'un pluriel trompeur. Et pourtant on ne peut objectivement passer sous silence le fait qu'ils sont massivement constitués de femmes qui font leur force et leur efficacité.

En préalable, une très rapide présentation des mouvements populaires d'habitation à São Paulo s'impose. Cette ville, la plus grande d'Amérique latine, connait comme toutes les mégapoles du monde des problèmes aigus de logement rencontrés par les classes populaires. Il y a aujourd'hui un déficit d'environ un million de logements ou, ce qui est plus exact, il existe un million de familles qui ne reçoivent pas des salaires suffisants pour acheter cette marchandise qu'est une maison. Ces familles vivent dans des favélas, dans l'obsession du loyer auquel ils doivent consacrer entre 20 et $40 \%$ de leurs maigres revenus.

"A casa propria", (une maison à soi), est donc un rêve et une nécessité absolue pour ceux qui gagnent un salaire dérisoire, dont la trajectoire professionnelle n'offre aucune stabilité et dont la vie reste marquée par quelques éléments irréversibles comme la migration, le manque d'éducation scolaire. Rêve du repos - l'inquiétude des loyers en augmentation constante s'éloigne -, de la sécurité, rêve et lutte afin d'obtenir une vie plus digne, plus humaine.

Ce sont dans ces conditions objectives et subjectives - l'on pourrait dire affectives - que sont nés dans les années 70 les mouvements populaires et dans les années 80 les mouvements d'habitation. Ils revendiquent entre autres la garantie du droit d'usage de la terre pendant 99 ans, le financement des infrastructures urbaines par l'Etat ou la Municipalité, mais surtout le droit de construire en mutirâa (groupe de construction autogéré) afin d'avoir des maisons moins chères. Le temps de la lutte puis celui de la construction est vu comme un temps propédeutique où l'on peut acquérir les bases d'une nouvelle compréhension de la société et de la vie en société fondées sur la justice. Deux concepts opératoires sont à l'oeuvre: la participation et l'égalité, entre les hommes, entre les hommes et les femmes.

Qui s'inscrit dans un mouvement populaire d'habitation? Majoritairement, des migrants de première génération, ayant une formation scolaire très faible ou même inexistante, exerçant des métiers très peu qualifiés. Si $50 \%$ des femmes sont mariées, $30 \%$ sont chefs de famille; les autres $20 \%$ sont divorcées, séparées, veuves ou vivent en union souvent instable avec un compagnon. Ces femmes sont souvent domestiques ou travaillent à la journée, gagnant entre un et un salaire et demi minimum; pour elles, la construction d'une maison en mutirâo est la seule chance de sortir d'une situation souvent trop difficile. Faut-il souligner que les valeurs symbo- 
liques liées à la stabilité d'un foyer, fondamentales pour les enfants, sont vécues avec beaucoup plus d'intensité par les femmes que par les hommes.

Le Mouvement d'Habitation de Vila Remo, zone sud de São Paulo, avec lequel j'ai travaillé ${ }^{1}$ ne fait pas exception à la règle. Les femmes dont $30 \%$ sont chefs de famille y assument de nombreuses tâches: présence aux Assemblées générales tous les 15 jours, coordination de petits groupes de 25 familles, présence dans les diverses commissions constituées durant la période de lutte (négociation, santé, éducation, etc.), responsabilités au secrétariat ou ménage. Ce sont encore elles qui assurent le succès des diverses manifestations de revendication comme les défilés dans la rue et les campements d'une durée de 6 à 10 jours, non seulement par leur nombre mais aussi par l'accomplissement des "tâches élémentaires" comme la préparation et l'organisation des repas, la surveillance des enfants, etc. Il faut souligner que cette participation intense des femmes repose, semble-t-il, sur deux présupposés qui se renforcent l'un l'autre et dont "l'évidence" n'est jamais remise en question:

- On admet parfois que les femmes font de nombreux travaux; les travaux non payés (ceux de l'entretien de la maison et de ses habitants) et payés car elles sont nombreuses à exercer des activités informelles afin de pouvoir, justement, faire les deux et apporter l'argent nécessaire à la survie du foyer. On voit là, à l'oeuvre, la conviction en la déqualification du travail féminin qui résiste aux faits les plus évidents.

- Le deuxième présupposé est celui qui consiste à dire - et à affecter de croire - que les femmes "n'ont rien à faire", qu'elles ont du temps à perdre et que, par conséquent, elles peuvent et doivent participer aux activités des mouvements populaires. Là encore, la déqualification du temps des femmes est à l'oeuvre de manière flagrante mais aussi insidieuse.

Lorsque la construction des maisons en groupes commencera, elles effectueront exactement les mêmes travaux que les hommes. Mais elles effectueront manuellement, en plus, le transport des matériaux de construction et de l'eau, travaux considérés comme l'extension des travaux ménagers et par conséquent comme "féminins" et non difficiles (or le terrain était en forte déclivité et l'eau peut être lourde). Seule la manipulation de la bétonneuse, unique objet technique du chantier problablement considéré "d'essence masculine", est réservé aux hommes; mais ce sont les femmes qui l'alimentent en apportant les éléments nécessaires à la fabrication du béton.

Même si les femmes font avancer le chantier au même titre que les hommes, et parfois davantage car leur présence est souvent plus régulière, elles n'assument pas de responsabilités au sein des groupes techniques qui sont constitués et les relations hiérarchiques hommes-femmes s'exercent toujours de la même manière. Néanmoins, elles sont conscientes d'avoir assumé correctement tous ces travaux de construction considérés comme "un travail d'homme" et elles en sont très fières. Comme le reconnaît le leader du Mouvement, "la femme a la volonté de réussir des choses nouvelles. L'homme pense que c'est bien comme c'est, que les enfants vont grandir comme lui; la mère souhaite d'autres choses pour ses enfants, qu'ils aillent à l'école, qu'ils aient un meilleur emploi." Il ne s'explique pas la plus grande participation des femmes mais il la reconnait et sait parfaitement bien s'en servir.

1. Ma recherche fut menée de 1986 à 1990 dans le cadre d'un accord CNRS-Orstom. 
Si l'on essaie de faire une évaluation rapide, un certain nombre de constatations s'imposent:

- La participation a permis à beaucoup de femmes de sortir de l'isolement de leur maison, isolement où les tient la peur qui règne dans les favélas (peur de la drogue, des violences, des meurtres et donc une méfiance très forte envers les autres, les voisins). Elles apprennent à nouer de nouveaux liens sociaux, des liens de convivialité qui permettent de travailler, de s'amuser ensemble, d'échanger: "J'étais révoltée. Depuis que je suis entrée dans le mouvement, que je partage avec les autres qui sont pour moi comme une famille, des parents, que je m'intéresse à eux, que je parle avec eux, je me suis ouverte. C'est bon pour ma vie."

- Elles ont accès à l'espace public, à des actions nouvelles, à la construction de l'affrontement entre la société civile et l'Etat, fondé sur la reconnaissance et la revendication de leurs droits de citoyennes. Prise de conscience qui suppose du temps mais qui leur donne confiance dans leurs possibilités qu'elles ne soupçonnaient pas, dans leur adaptation à de nouveaux rôles. "Quand on participe à quelque chose, cela devient une partie de soi. Ce fut très intéressant pour ma vie. Je ne sais comment t'expliquer. Le travail de construction fatiguait beaucoup mais le jour suivant je ne voyais pas l'heure. Physiquement j'étais en dessous de tout mais psychologiquement je me sentais très bien parce que si tu ne participes pas, tu te sens inutile. Tu as réfléchi? J'ai une maison ici, j'ai aidé à faire le ciment de cette maison, les fondations. Je l'ai fait. Tu sais ce que c'est 'moi'? Ce moi des personnes qui est rempli de vie."

- Le fait d'avoir enfin une maison décente (53 mètres carrés) qui les met, elles et leurs enfants, à l'abri du cauchemar de l'expulsion à cause d'un loyer trop cher et leur donne un sentiment d'ascension sociale et la conviction d'avoir contribué à cette amélioration fondamentale de leurs conditions de vie. Elles ont gagné une certaine confiance en soi, elles ont découvert qu'elles peuvent construire une action politique pour elles et pour les autres, tout aussi démunis.

Elles ont appris qu'elles ne sont pas seulement des mères, des ménagères mais aussi, comme elles le disent si souvent, "des personnes", ou comme nous le disons, "des citoyennes". Mais l'appartenance à un mouvement populaire a une fin; dans ce cas précis la fin est arrivée après 4 ans ( 2 ans de lutte et 2 ans de construction). Ce temps est-il suffisant pour forger définitivement une nouvelle personnalité qui puisse échapper aux pesanteurs culturelles de la société? On ne peut l'affirmer. Formulons pourtant l'hypothèse que cette expérience si profonde ne peut que laisser de straces, en elles-mêmes, dans leur vie et dans ce qu'elles seront amenées à transmettre à leurs enfants. On peut légitimement espérer que, si de nouvelles occasions de lutter surgissent, elles seront les premières à y entrer, renforçant ainsi leur expérience et leurs acquis politiques.

Pour conclure, il faut souligner un trait qui est de plus en plus mis en exergue par les recherches sur les femmes en milieu urbain, quels que soient le continent et le pays. Par leur travail à la maison, par leur acceptation de n'importe quel travail rémunéré, par leur engagement et leur participation dans des actions collectives dont l'objectif est d'améliorer les conditions de vie, ce sont les femmes qui permettent aux Etats de continuer leurs politiques de désengagement social. C'est une raison de les admirer; il faut pourtant voir le danger que cela représente, pour les femmes mais aussi pour les sociétés. Tresser des couronnes aux femmes permettra peu à peu aux gouvernements de pouvoir esquiver, en toute bonne conscience, les devoirs qu'ils ont envers les plus démunis. 


\title{
LA MAISON DES AUTRES, «A CASA DOS OUTROS»: L'EMPLOI DOMESTIQUE A RECIFE, NORDESTE DU BRESIL
}

\author{
Marie ANDERFUHREN
}

\section{Généralités}

L'emploi domestique est la première occupation féminine rémunérée des zones urbaines du Brésil, puisqu'elle occupe grosso modo pas moins de $24 \%$ des femmes actives. Or l'insertion de ces femmes dans la vie active et dans la vie urbaine est assez particulière, puisqu'une grande partie commence cette vie en habitant sur le lieu de travail, comme elles le disent «dans la maison des autres». Pour les migrantes venues en ville en vue d'obtenir un emploi (la moitié des migrantes que nous avons interrogées, les autres, toute jeunes, migrant avec leur famille), le fait de résider dans la maison des patrons est un passage obligé. Le texte qui suit traite de ces femmes migrantes qui sont venues en ville en quête d'emploi.

\section{Autres modes de consommation}

L'approche de l'environnement urbain et du travail est donc particulière, puisqu'elle se fait en entrant dans l'intimité d'une classe sociale différente, aux modes de consommation foncièrement différents, modes de consommation que l'employée domestique devra «gérer», mais auxquels elle n'aura pas pleinement accès. Nous verrons tout d'abord ce que signifie cette gestion particulière de la consommation des autres, et lorsqu'il n'y a pas gestion, cette inévitable confrontation ou du moins ce saut dans un mode de vie nouveau. Elle nous semble caractéristique de cette migration féminine et la différencie de la masculine, où cette confrontation bien que brutale n'est pas aussi immédiate.

\section{Les alliances}

Ce n'est pas seulement la question de l'inévitable confrontation quotidienne à des modes de consommation autres que les siens qui fait la différence entre une migration masculine et une migration en vue de trouver un emploi domestique, mais c'est également la proximité de personnes alliées ayant de l'influence qui permettent, voire permettront, au moment venu, de faire le pas de vivre dans sa propre maison. Même lorsque le mari fournit la maison, l'appui d'une patronne est recherché. La recherche souvent frustrée de la bonne patronne n'est pas étrangère à ce besoin de trouver des appuis indispensables pour son insertion en ville, hors de la maison des patronnes.

La construction de liens avec des patronnes et leurs familles est parfois analysée par les employées domestiques comme de véritables calculs, un mode d'échange, un moyen d'obtenir des avantages. Ce n'est pas toujours le cas loin s'en faut. La mise en place d'attitudes devant ultérieurement permettre d'obtenir une amélioration de son sort signifie que l'on a déjà fait le pas qui consiste à penser que l'on mérite mieux, que l'on sort en quelque sorte de sa propre stigmatisation. Or la stigmatisation dont fait l'objet l'emploi domestique est d'autant plus forte qu'elle est souvent partagée par les principales intéressées. 


\section{La chambre de bonne}

Cette recherche d'un lieu d'habitation propre est une constante du discours de l'employée domestique, même pour celles qui font le calcul de ce qu'elles perdront en termes de confort et de revenus non monétaires si elles vivent hors du lieu de travail. Mais cette constante du discours ne signifie pas pour autant que toutes les pratiques se dirigent vers l'obtention d'un logement autonome; car dire que l'on est dans une situation frustrante et insatisfaisante ne signifie pas que l'on mobilisera des énergies pour pouvoir sortir de cette situation. Encore faut-il croire que l'on peut en sortir, c'est-à-dire, entre autres, se penser comme individu, comme genre et comme catégorie sociale, digne et capable d'accéder à une condition meilleure que celle de domestique résidente. Sans entrer dans les détails, on peut avancer que la construction de stratégies est autant liée aux conditions objectives d'accès à des emplois meilleurs - voire à d'autres situations de vie - qu'à la construction quotidienne de son identité. Comme le montre de manière éloquente une enquête menée à Recife sur la chambre de bonne, le lieu où résidera cette bonne est à l'image de la relation sociale qui caractérise l'emploi domestique. Ce qui marque l'emploi domestique c'est l'invisibilité. La chambre est toujours un lieu d'isolement par rapport aux autres habitants. Il est exigu, il sert souvent à la fois de chambre et de dépôt. Il peut même être réversible : chambre à coucher la nuit, chambre à tout faire le jour. A l'image de l'employée domestique elle-même dans la famille, il est préférable que sa chambre ne prenne aucune place dans l'habitation, car il y a toujours le risque, plus fantasmé que réel, que l'employée se mette à confondre les rôles (crainte souvent mentionnée par les patronnes que nous avons interrogées).

\section{Gestion et exécution}

Confondre les rôles c'est croire que l'exécution répétitive autorise à prendre des initiatives plus larges que ce qui est strictement autorisé par la patronne: changer les meubles, faire des achats inconsidérés, avoir un avis ferme sur l'éducation à donner aux enfants; ce que craint la famille employeuse c'est que l'employée se prenne pour la patronne, car, quelle que soit l'autonomie laissée à l'employée pour l'exécution des tâches, l'initiative et la responsabilité finales incombent à la patronne. Lors de garde d'enfants la délimitation des rôles est étonnamment fixe, si l'on songe à la charge affective que comprend nécessairement ce travail: il n'y a pas de confusion entre la bonne et la mère, il ne doit pas y en avoir.

L'employée domestique, quand elle arrive dans son premier travail, est souvent utilisée comme gardienne d'enfants ; bien que, aujourd'hui, on retrouve de plus en plus de très jeunes filles faisant tout, une bonne partie de la classe moyenne ne pouvant plus se permettre d'avoir plusieurs employées. ${ }^{1}$ Ce n'est que petit à petit qu'une employée pourra se voir confier de plus en plus de tâches de la maison, cuisine incluse, des tâches telles que les payements à la banque, les achats, etc. Cet état de faits, même s'il est relaté comme la preuve par excellence de la confiance et de la réussite, est rare. Plus nombreux sont les cas où la responsabilité de la gestion du ménage demeure entièrement du ressort de la patronne, quelque libérale qu'elle soit.

1. La paupérisation de la classe moyenne entraîne le double phénomène de l'apparition de bonnes à tout faire là où il y avait cuisinière, gardienne d'enfants, femmes de ménage, etc., et paradoxalement une spécialisation (assez lente dans le Nordeste): on voit apparaître des lavandières dans des ménages où l'épouse s'est mise à faire elle-même la cuisine à la place d'une cuisinière que l'on ne pouvait plus payer, ainsi que les nettoyages quotidiens, mais où une tâche reste confiée à une employée domestique spécialisée. 
Etre bonne d'enfants signifie que l'on n'a a pas directement à gérer le ménage, l'utilisation de la nourriture et des produits de lessive. De toute manière, les migrantes apprendront tout de leur patronne. Rien de ce qu'elles savaient faire auparavant ne semble adéquat; du moins le ressentent-elles ainsi. La question est de savoir ce qui provoque cette dévalorisation systématique de ce qui précède l'emploi domestique, alors même que l'emploi domestique lui-même est très mal vu. Nous pensons, quant à nous, que c'est un des multiples mécanismes mis en place pour entrer dans le monde urbain, pour supporter le choc, pour finalement essayer d'en tirer profit. Voyons de plus près quel en est le fonctionnement.

\section{L'avant-migration dévalorisée}

La vie avant la migration est dévalorisée. Il n'y a pas lieu ici d'entrer dans une description des différentes manières dont est établi le contact avec la première employeuse et par conséquent logeuse. En revanche, il convient de relever que, alors même que l'emploi domestique est l'objet d'une stigmatisation, et pas des moindres, la vie avant la migration est également dévalorisée. La construction d'une identité de femme urbaine doit probablement passer par cette dévalorisation; d'autre part un passé très dur, lorsqu'il est reconnu comme tel, permet de supporter un présent qui parait, en comparaison, plus acceptable.

\section{Cette dévalorisation se manifeste de différentes manières:}

- Tout d'abord il est très courant chez les employées domestiques migrantes (relativement peu nombreuses à passer par une petite ville avant l'arrivée à Recife) de juger qu'à leur arrivée en ville, elles n'étaient que des bobas do Interior, des Bécassine de la province ou de la campagne. On explique ainsi à la fois la raison pour laquelle on a été exploitée sans pouvoir réagir, et pourquoi on s'est sentie perdue en ville. Certains entretiens s'attardent passablement sur cet aspect, tout de même compliqué à gérer, surtout lorsqu'on est analphabète. Mais la dévalorisation de ce que l'on était permet de mesurer le chemin que l'on a parcouru. On peut dire que la ville rend experte, «actualisée» comme elles se plaisent à dire. Experte dans toutes sortes de domaines dans ses rapports de travail, dans sa capacité de lier des amitiés utiles, dans la manière de s'y prendre avec les hommes, etc.

- Ensuite cette dévalorisation se manifeste par la place que les migrantes accordent à leur famille, à leur mère en particulier dans l'apprentissage des tâches ménagères. Non seulement la majorité dit avoir tout appris de la ou des premières patronnes (ce qui n'est pas le cas pour les employées domestiques nées en ville qui, beaucoup plus souvent, disent avoir acquis un savoir-faire au travers de leur mère), mais encore elles font extrêmement peu de cas de leur premier apprentissage du travail, alors que la description de ce travail, celui des champs la plupart du temps, montre à quel point il est rude.

«Chez ma mère, je travaillais mille fois plus que chez ma patronne» est un commentaire fréquent. Il fait partie intégrante de cette valorisation de l'urbain, assez paradoxale si l'on songe qu'en même temps les domestiques sont à peine mieux que des «videuses de pots de chambre», des piniqueiras.

Les entretiens révèlent toutes sortes de détails sur ces premiers pas, pour le moins gauches, dans le travail domestique. Ils sont corroborés par les récits des patronnes, qui, même lorsqu'elles préfèrent des jeunes filles de l'intérieur, plus malléables, ayant la réputation d'être plus honnêtes et dont les réseaux permettant leur contrôle sont parfois sophistiqués, ne manquent pas de relever leur inadaptation à la modernité, aux électroménagers, aux repas urbains, etc.

Finalement, on ne peut s'empêcher de se demander: pourquoi l'employée fait-elle un tel hommage, au passage, à la bonne patronne qui lui a tout enseigné? De puissants mécanismes d'identification existent chez de nombreuses employées. 


\section{La conquête de la ville et l'enfermement}

L'extraordinaire étrangeté de la ville est vaincue de diverses manières. L'une est de suivre sans défaillance les patronnes, voire leurs enfants, dans tous les déplacements. L'employée ira au Shopping Centre avec la patronne, ainsi qu'à la plage, etc.; on apprend la ville dans un premier temps au travers des activités de ses patrons, surtout si l'on arrive très jeune.

Mais l'approche de la ville est souvent beaucoup plus conflictuelle, car si une patronne choisit une fille de la campagne que l'on pense en général plus honnête, plus pure, plus malléable, moins revendicatrice, ce n'est pas pour qu'elle s'urbanise ou s'encanaille avec des consoeurs de la ville. Nombreuses sont les employées domestiques qui racontent l'enfermement, l'interdiction de sortir, et les méthodes qu'elles inventent pour contourner cette interdiction, qui peut entraîner des départs subits. Ils sont expliqués souvent par l'accord passé entre les parents de l'employée domestique et la patronne, accord qui implique un contrôle auquel l'employée échappe lorsqu'elle sort. Parfois cet accord stipule l'interdiction de sortir: «Si j'avais parlé de sortir, ma patronne m'aurait tuée, parce que ma mère lui avait recommandé de ne pas me laisser sortir, même pour aller à la boulangerie», explique une employée.

Une autre ajoute de manière plus générale : «Ma patronne, elle avait peur de ma mère, du reste de mon père aussi, parce que ma mère, ces personnes de la campagne, quand une de leurs filles va travailler, elles la confient comme s'il fallait qu'on prenne soin d'elle; quoi qu'il arrive la responsabilité incombe à la patronne.»

On entre du coup dans un schéma que l'on retrouve dans une moindre mesure chez les nonmigrantes, où l'extérieur, le dehors, la ville est le lieu de tous les dangers. Pêle-mêle on peut citer pour les patronnes: la crainte que l'employée tombe enceinte, qu'elle fasse venir l'extérieur à l'intérieur de la maison (notamment qu'elle fasse venir des hommes ou, dans une moindre mesure, des copines dans l'appartement), la crainte que l'employée se transforme en urbaine revendicatrice, c'est-à-dire étrangère par excellence, voire en voleuse. Certes toutes les employées ne vivent pas le même degré d'enfermement, loin de là, et la liberté de mouvements est de manière générale beaucoup plus grande pour une employée travaillant chez des employeurs sans ressources que chez les riches. D'autre part certaines sont encouragées à aller à l'école, car la bonne maitrise des tâches domestiques passe par une maitrise de l'écriture, parce que l'école, à tort ou à raison, est un lieu de socialisation qui est bon, sans trop de dangers, limité, et où l'employée ne "se perd pas». Finalement beaucoup de patronnes semblent trouver bon que l'employée aille à l'école sans trop donner de raisons, l'école étant probablement une valeur de la société brésilienne assez largement répandue.

D'autres employées domestiques sont chargées de certaines tâches qui, une fois accomplies, les laissent libres jusqu'au retour des patrons entre 5 et 6 heures du soir.

\section{La grossesse}

Une des grandes craintes liées à l'extérieur est celle de la grossesse. Plusieurs patronnes racontent que, dans ce cas-là, elles ont congédié leur employée, car il est exclu que l'employée «parasite» son temps de travail par du nursing, voire contraigne la patronne à donner des soins.

Il est remarquable de recenser dans les entretiens, l'importance que prennent les mises en garde des patronnes par rapport aux fréquentations masculines. Les employées le relatent souvent lorsque, après coup, elles ont l'impression que la patronne avait vu juste. Mais cette opinion 
(forte) donnée sur les fréquentations qu'a l'employée domestique est un point d'accrochage pouvant entraîner des ruptures.

Les grossesses ne sont pas toujours des temps d'exclusions, et les conseils peuvent tout aussi bien être d'avorter comme de déconseiller un avortement. Les patronnes ne renvoient pas forcément l'employée, même si c'est le cas de figure le plus fréquent. Certaines patronnes acceptent que l'employée garde son enfant avec elle. (Il est remarquable de constater que les employées relatent des expériences où elles cherchent un autre type de travail qui ne leur serait accordé que si elles se faisaient stériliser, par ligature des trompes. Or ces exigences ne sont jamais relatées pour la recherche d'une place dans l'emploi domestique.)

La grossesse peut être u $n$ moment charnière de l'insertion en ville hors du lieu de travail, car c'est souvent le moment où il s'agira de concrétiser son envie et la nécessité d'avoir sa propre maison. Plusieurs scénarios existent: l'employée cache sa grossesse le plus longtemps possible; elle a un premier enfant; elle continue à vivre chez sa patronne; sa patronne l'aide.

L'employée place le compagnon ou le mari dans une situation où celui-ci se met (parfois) à chercher un logement: une chambre louée, un lieu dans sa propre famille. Le premier moment de vie hors de l'emploi n'est pas toujours définitif, c'est souvent une situation précaire, parfois si précaire même qu'il faut redemander à d'anciennes patronnes de revenir en résidente travailler, quitte à envoyer l'enfant dans sa famille d'origine ou à le confier à d'autres tiers. Car tout dépend: le compagnon travaille-t-il? A-t-il de quoi payer le loyer d'une chambre? La patronne supporterat-elle les changements tout de même très importants qui auront lieu dans le mode de service offert par l'employée domestique si celle-ci se met à vivre à l'extérieur?

C'est là que les liens établis avec la patronne, voire avec les patronnes précédentes, vont être cruciaux. Ils permettent des emprunts (en argent) ou des arrangements pour le matériel de construction, pour les meubles, les lits, les tables, etc.

La conscience est parfois très claire que les liens avec la ou les patronnes seront déterminants pour l'accès à un terrain. Ces liens permettant ou facilitant l'acquisition d'un lieu propre sont d'autant plus importants quand le mari et la famille du mari sont peu à même de fournir l'argent pour une location, voire une chambre dans une maison déjà existante, etc.

«Si j'avais un homme qui m'aide, je pourrais épargner un peu, mais comme ça je ne peux pas. Pour acheter un terrain, je demande de l'aide à l'une ou à l'autre de mes ex-patronnes, j’ai encore des contacts avec elles, je sors leur demander. A celles avec qui j'ai le plus de contact, à Dona...»

Cette constitution d'appuis dans une autre classe sociale, chez ses patrons, pour parvenir à sortir de l'emploi sans être à la rue, pour passer d'employée résidente à non résidente, ne réussit pas toujours. Loin de là. Le mariage est peut-être une solution plus simple, quoi-qu'il soit loin d'être facile d'avoir un compagnon qui ait un travail, afin d'ajouter à son salaire celui de son mari. Et les histoires où le salaire de l'employée constitue l'essentiel des gains sont légion.

Que fait-on lorsque les alliances avec les patronnes n'existent pas ou sont insuffisantes? Ce cas est tout de même extrêmement fréquent. Et que que fait-on si l'on n'a pas trouvé de compagnon chez qui l'on puisse vivre? Souvent on reste résidente et l'on passe d'une place à l'autre en quête de la bonne patronne. Si l'on a des enfants, on les fait élever par sa mère, ses frères et soeurs ou 
d'autres tiers. Les cas ne sont pas rares. On peut vivre dans un isolement considérable. On peut aussi chercher des appuis ailleurs. Les Eglises peuvent être un de ces appuis. Il est difficile d'en évaluer la portée. Elles constituent des réseaux, permettant notamment de trouver du travail. Elles peuvent parfois être des pourvoyeuses d'aide directe, ou faciliter certaines invasions de terrain.

Lors d'organisation d'invasions de terrains, on peut même trouver des appuis dans le voisinage immédiat de l'invasion, voisinage qui, pour diverses raisons, trouvera avantageux d'avoir, à la place de marais insalubres, un début d'urbanisation si sauvage soit -elle.

A première vue pourtant, pour sortir de la situation de résidente dans l'emploi domestique, pour acquérir sa propre place en ville, les réseaux secondaires - Eglise, voisinage, association d'habitants - semblent plus de «second recours» que ces alliances liées à l'emploi et au mariage. Ils sont pourtant constitutifs de cette socialisation multiple que les employées domestiques résidentes et migrantes ne semblent pas toujours à même de construire, et qui pourtant facilitent l'insertion en ville, hors de «la maison des autres». 


\title{
THE THIN EDGE OF WEDGE-PUSHING ACCOUNTABILITY INTO PUBLIC SYSTEM. EXPERIENCES FROM BOMBAY
}

\author{
PRATIMA PANWALKAR
}

\section{A REFLECTION}

The U.N. Decade for the "Advancement of Women" has pushed the agenda for a "Woman Focus" into development planning of all Third World countries. This preponderance of the "women in development" (WID) thrust was picked up by many country governments and international donor agencies, both bilateral and multilateral.

The organizational response to this has been the creation of "purpose built institutions", like women's bureaus, ministry for women and women's departments, a softer approach than strengthening the woman focus in existing agendas. This sector suffered the same ills, as the group they sought to serve - ill-staffed, underfunded and marginalized. "The conceptualization of both the problems and strategies of this sector in terms of women, not gender..."1 persists to be the Achilles heel of these efforts till today.

Development activists working in the field both in urban and rural areas have been more cautious, taking their own time to pick up this intellectual baggage. Rooted in the socio-cultural reality of the development models accentuating poverty, the lack of access to basic facilities and resources and an insensitive bureaucracy seem to be the more critical issues to be addressed to, affecting both men and women in the communities. The special burden of women had to be unfolded at many layers within self, at family and community level and in interaction with systems. The persistence has paid off and in the 90's "gender sensitivity" has replaced "women focus", as the major paradigm for action.

This paper outlines the experience of women from slum settlements in Bombay, who in the process of negotiating with the system are recreating for themselves new patterns of relationship at all levels.

\section{A LOOK AT BOMBAY}

As one of the major metropolis in the Third World, Bombay's population now at 12 million is recorded to have almost $50 \%$ of its population living in about 3000 identified slum pockets. This

\footnotetext{
${ }^{1}$ LEVY Caren, "Gender and the Environment: the challenge of cross-cutting issues in development policy and planning", Environment and Urbanisation, Vol. IV, No. 1, April 1992.
} 
sprawl is the direct outcome of a market oriented land economy, where the annual formal housing shortage is almost 15,000 units. The slums in the city occupy lands belonging to Central and State Governments, Municipal authority and private individuals. This to a large extent determines the scope and nature of public agency intervention in these communities.

The approach of the State and local authorities to these spontaneous settlements swings between indifference to a range of parallel and simultaneous strategies which can be classified as (i) improvement, (ii) upgrading, (iii) redevelopment, and (iv) resettlement. In the evolution of the first three strategies, one can perceive a somewhat logical and gradual progression of thought and action. This in no small measure has been the contribution of the universal approach to spontaneous settlements as reflected through international funding and consequent changes in the policies of country governments. The last approach i.e., of resettlement, is being used extremely judiciously and in exceptional cases.

Of course as is the common phenomena striding all this, is the patent bureaucratic indifference and "ostrich-like" posture which does not allow any "proactive" action to emerge, and prompts a favoured "line of least resistance", in a sector that is politically and socially volatile and administratively extremely complex. (see Annex I)

\section{BACKGROUND OF THE COMMUNITIES}

Shivaji Nagar Colony is one of the resettlement areas created by the Municipal Corporation of Greater Bombay, to rehouse families who were shifted from their original residences, since the year 1969. It comprises three major pockets i.e., Lotus Colony, Baigan Wadi and Shivaji Nagar. Each of these pockets have been further subdivided in plots and 114 such plots exist in the area, with a population of about 150 thousand. The resettlement colonies represent well planned lay-outs, with a well laid out internal road structure and basic amenities of water, toilets, and drainage system provided in each plot. The residents have obtained lease hold rights on the plots they occupy and thus there is no fear of eviction. The colony has also been provided with four primary and middle level schools, one urban-health centre run by the Corporation, and a post-office. There are numerous ration shops, which serve as sale points for the goods sold through the public-distribution system. Numerous local markets and commercial establishments exist, specially along the main roads, which serve the daily needs requirements of the residents. The city bus transport system has a major terminus in the area and the suburban railway station is at about 15-20 minutes walking distance from the community. Numerous privately managed health and educational services have also sprung up in the area to cater to the community needs.

Availability of open land and of basic infrastructures located in the resettlement colony has resulted in an unchecked development of spontaneous settlements on the periphery of the colonies. Over the years these settlements have grown in numbers and today there are about 15 such identifiable communities dotting the borders of the colonies with an almost equal population of 150 thousand residing in the area. Being totally unplanned and unauthorized these communities have not been provided with any basic amenities, thus resulting in pressures on the existing facilities in the colonies, leading to a near total breakdown of the existing systems. Some efforts have been made through political patronage to obtain basic water and sanitation amenities, but the success has been very marginal. 
As both the communities have grown at different points of time and the residents are from different places of origin the area today reflects a very heterogeneous and mixed population composition. The dominant religious group residing in the locality is Muslim (about 60\%), with Hindu, Sikhs, some Christians and Neo-Buddhists accounting for the remaining. They are also drawn from different regions of the country. The growth of the peripheral slums reflects the internal growth of the families in the resettlement colonies and their success in engaging in land speculation in the unguarded open plots around the colony and so there are definite familial and community ties between the residents of the resettlement and slum colonies.

Typically the two settlements also reflect the difference between consolidated and peripheral areas in economic activities engaged in by the residents. Most of the plot residents are employees of organized sectors like the state and civic bodies, and industrial units. They may have their own petty trades and businesses. There is a thriving home based Jari embroidery activity ("Jari" being the gold/silver threads). Many are engaged in self-employment activities contributing to the service sector of the economy. In the slum pockets however, most workers are daily wage earners, mainly contributing to the unskilled unorganized urban labour force. Yet this difference in income levels does not reflect very radically on other social indicators in the area, like health, levels of literacy, etc. In these fields it is uniformly seen that all the major killer diseases are present and water borne diseases are rampant. Women and children are specially susceptible to the range of health problems of the urban-poor, resulting from the inadequate water, sanitation and basic health care facilities. Low level of literacy exists in both the areas, specially among the women and though school enrollment is fairly high, there is also a high rate of drop out from the formal schooling. In the "Jari" units the children from the poor families are bonded to the unit owners.

\section{SOCIAL DYNAMICS OF THE SETTLEMENTS}

The heterogeneous nature of the population and their settlement patterns at different points of time have resulted in well identifiable community, with a general tendency to function in isolated manner. As characteristic of marginalized populations, the majority of their residents have a general tendency to keep to themselves managing the rhythm of daily life struggles and tensions. Interactions in small groups or at community level are more or less restricted to the social, cultural or religious spheres of life. There is a general mistrust of any new person or idea and people would rather wait and watch than give commitments that would disturb their established pattern whether in behaviour or attitudes they adhere to.

\section{ENTRY OF APNALAYA}

As a part of its layout plan, the Corporation had retained a small plot for a Multi-Purpose Community Centre in the Lotus Colony. After some unsuccessful efforts with some agencies, the Corporation was actively exploring for a NGO to undertake the responsibility of utilizing the premises. This was the year 1976 and during this period Apnalaya had stabilized its own position and had started engaging professional staff on its team. Earlier work experiences had convinced the agency that unless development is people centered with the community undertaking a major role in its growth, no change can sustain itself in the field. The agency was very keen to test out this approach and this was an opportune time to start work in the area, with a major commitment to people initiated change. Thus began the process of partnership of the agency with the communi- 
ties that have nurtured local groups into action and sustained themselves in the true spirit of sharing, not only rewards but also frustrations and failures.

Over the years the agency had consolidated its interaction with the local groups - becoming one of the major referal points in the settlement for any initiatives in community action. In 1992, a major challenge faced the neighbourhood, when it was the scene of communal tension that had gripped the city. Apnalaya with its deep rooted contacts and through its network of local volunteers was able to perform a critical role at this juncture. The urgent imperative to provide immediate relief and work from a base closer to the affected population prompted Apnalaya to start health-clinics in three areas of the fringe settlements - Chikkalwadi, Sanjay Nagar and Indira Nagar. The aftermath of the riots saw Apnalaya playing a major role in the economic rehabilitation process and in negotiating with competent authorities on behalf of local groups.

At the field level, the centres at the fringes got consolidated and have become the base for a major community outreach programme in health. In keeping with its approach the agency started mobilizing the local women and soon a band of highly motivated women have come together and are keen to give their time as CHW (community health workers).

Preparations for this new role was assiduously facilitated by the agency staff through participatory training sessions. This whole process was also documented through participatory research and the chart (page 196) highlights the preferences of the women's group in two communities.

"There were some interesting outcomes. Health ranked forth as a concern in both groups and accounted for less than $16 \%$ in either group. Throughout our sessions in both Chikkalwadi and Sanjay Nagar, several very dominant women were insistent that women needed jobs in order to improve their lives and that of their children. Other women in the groups were supportive of their comments. However, when individuals ranked their own preferences, women's paid work became less important. It ranked fifth out of the top six issues in both groups. Our Sanjay Nagar group explained that if a woman had a good husband, one who did not drink or have other bad habits, he would share his salary fully with the family, and there would be no need for the woman to work. Women had very low expectations about the kind of work they could do, they never mentioned wanting to learn to read and write. Jobs that women could do include selling food or small items from a basket, small stitching or sewing jobs, carrying dirt at construction sites, or recycling waste materials from the dumping ground. They preferred to have a flexible job that they could do in the house.

Of more immediate concern to the Chikkalwadi women were infrastructure problems - lack of legal sources for water and electricity, a milk depot and well stocked ration shops. While the Sanjay Nagar group already had water and electricity, they were still concerned about the state of the toilets in their area. There were too few toilets for too many people. Consequently, they were always filthy."2

For the groups of women engaged in this exercise, their new role as community workers has released a vast resource of untapped potential. Coming primarily from poverty level Muslim families, the women had continuously suffered the strains of their disadvantaged status. The intensive

\footnotetext{
2 TOLLEY Betsy, RAO Nandini and BULANI Rita, Participatory Research in Bombay, India: Concerns in Health Apnalaya - Research to Action Projet, June 1994.
} 


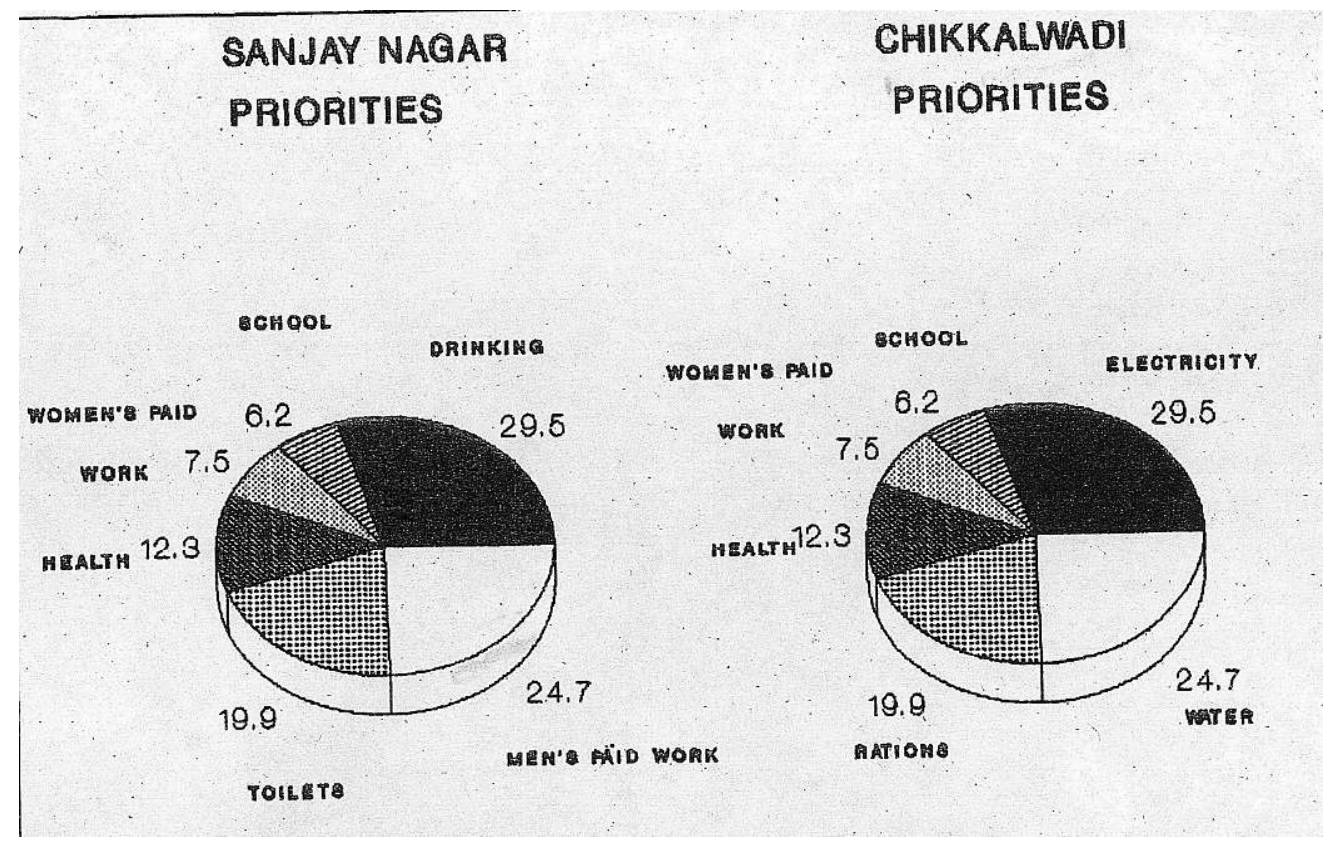

\section{CHART}

contacts and interaction with agency staff helped many of them look at themselves and their surroundings in a more proactive role. Their integration into the network of contacts that Apnalaya had at the larger neighbourhood level, opened up new possibilities that the women could direct their efforts into. Amongst others, one of the major areas of concern to the group was their anger at the state of the "ration-shop" that existed in the area, and to which the families from the fringe settlements were attached (see Annex II).

\section{Action at another level: entry into the Public Distribution System...}

The state of the Public Distribution System (PDS) and the ration-shops had always been a high priority issue for urban activists working in marginalized settlements. NGOs and CBOs spared no opportunity to put pressure on the system, representing the myriad problems that the ration-card holders faced. Towards the end of 1993, a major development took place at Bombay level that put out a ray of hope for these efforts. In the posting of a new Commissioner for Rations, the NGOs found an ally. With an earlier track record as a zealous and upright officer, totally committed to creating space for user groups involvement, this officer started exploring ways to shake the fossilised and much beleaguered system, riddled with inefficiencies and corruption. He sent out "feelers" to the NGO community to assist him in this task. Apnalaya, along with other NGOs, readily took up the challenge and soon a co-ordination committee of NGOs started meeting the concerned officers of the system, under the watchful eye of the Commissioner. Apnalaya with its earlier track record as a premier training agency in the city was also given the responsibility for conducting training sessions to infuse a "people-orientation" to the officers and staff of the various divisions of the Department. This process still continues. The once a month meeting, which continues with regularity, enabled NGOs to bring into sharper focus the problems in the different zones of the office. Joint delegation and protest marches have also been undertaken to focus on the issue at a city level. 


\section{... AND ITS IMPACT AT THE FIELD}

The area level committees created as a result of this persistent effort have been the scene of action for large group of residents, who have already been galvanized into taking responsibilities because of their continuous interactions with CBOs and NGOs. Widespread dissemination about the procedural requirements for ration-cards, ensuring periodic visits by rationing officers to the localities and most importantly creating and manning local vigilance committees have been the various activities engaged in.

\section{RESPONSE IN BAIGANWADI}

The women in Baiganwadi have also picked up the gauntlet in this struggle and formed a local area vigilance committee. In this they are learning from the experiences of Chikkuwadi, another community where Apnalaya has been instrumental in very active group formation on this issue, just 15 minutes walking distance from Shivaji Nagar. Of course, the task is not easy, the stakes involved in the continuous mismanagement of the system are very high. The six shops that serve the Baiganwadi area are located in Shivaji Nagar at a distance away from their immediate neighbourhood. It is known that these shop-keepers have pressed into service local "muscle-men" ostentatiously to keep "discipline", when the crowds get restive, but more to silence any dissidence. This poses a real threat to the women, who are physically in danger of being man-handled.

"Strength in numbers", both from within and outside the community is one major strategy engaged in by the active women residents. Interestingly here, local youth groups and residents' associations have also joined the effort.

In other parts of the city where the NGOs are active, similar experiences are emerging. Reports and presentation by active women residents are the order of the day in the monthly meeting. Media aids like posters, banners, street-plays and songs have been prepared and are used by all the activists in the field. A militant approach and urgency in action characterize their efforts. But the struggle cannot be fought alone. Is "rationing" a women's issue, because it is in the domestic sphere? Then so are issues like better water, toilets, schools, health, jobs where countless struggles are going on led and managed by women. The answer to this lies in the new self-identity of the marginalized urban woman and her ability to convert her skills and concerns as an instrument of negotiation and bargain.

The imperatives of the social reality in which women are forced to exist, the utter callousness of concerned authorities towards this and the opportunities provided to wider exposure through CBO and NGO networks have all contributed to the making of this identity. Dilemmas within it are plenty. What is considered the "right" thing to be done? and at what "cost"? Is women's activism a monopoly of the young, the separated or estranged wife, who has no other responsibility? Is the stereotyping to be fought against at all socio-economic levels? How to tackle the adverse impact at family and neighbourhood level, where new roles challenge expected demands? Is the adjustment possible at all levels of the family?

For the NGOs and women as they seek the answers to these questions through their life experiences, a continuous vigil has to be kept at the systemic level to locate spaces that allow them to act. This by itself is again governed by various factors, the stranglehold of vested interest and degree 
of bureaucratic initiative being crucial. Pressure from the hustings has to be consistent and continuous.

\section{CONCLUSION}

Maharashtra State, where Bombay is located, prides itself in being amongst the most progressive and industrialized States. It has also been the seat of the women's movement in the country both at intellectual level and activism in the field, for almost a century. In June 1994, the Government of Maharashtra has declared a "Policy for Women", the only State in the country to do so. Though claimed as revolutionary, this document has only brought together a range of initiatives that have taken place and outlined a new "thrust" and "vision" to them. For activists at grass root level both from within and outside the community, the pressure is now on for a shift from the "private" to the "public" domain.

\section{ANNEX I \\ Statistical Profile of Bombay City \\ (drawn from available data with BMRDA)}

Area:

(original island) Bombay's area

BMR region area

Greater Bombay area

Bombay population

(1951)

(1981)

Male Female ratio $=$

Population:

Density 16,400 per sq.kms.

Slum population more than $50 \%$.
$78 \mathrm{sq} . \mathrm{kms}$. 4375 sq. kms. $603 \mathrm{sq} . \mathrm{kms}$.

2.9 million

8.2 million (1991)

9.9 million

1000:820

Slums:

$\begin{array}{lll}\text { In } 1976 & 1350 \text { slums } & 0.63 \text { million huts } \\ 1990 & 2165 \text { slums } 1.1 \text { millions huts } \\ 1990 & \text { Pavement Dwellers } 25,000 \text { households }\end{array}$

The number of protected huts is almost 2 times the number of unprotected huts in censused area, i.e., $50 \%$ increase in the number of huts on notified lands. There are no new encroach-ments on unnotified lands.

Lands under Slum Settlements:

Lands

12.75 sq. kms.

$3.93 \mathrm{sq} . \mathrm{kms}$.

$4.60 \mathrm{sq} . \mathrm{kms}$.

$1.63 \mathrm{sq}$. kms.

$2.23 \mathrm{sq} . \mathrm{kms}$.
No. of Huts in Percentage

$50.38 \%$ on private lands $15.59 \%$ on state govt. lands $18: 19 \%$ on BMC lands $6.46 \%$ on Central Govt. lands $8.82 \%$ on $\mathrm{MHB}$ lands 
$0.13 \mathrm{sq} . \mathrm{kms}$ :

$25.27 \mathrm{sq} . \mathrm{kms}$
$0.52 \%$ on unidentified owners' lands

Total lands covered by huts

There are 1.1 million huts covering the total land area of 25.25 sq. $\mathrm{kms}$. or approximately $4.1 \%$ of the total city land is occupied by about $50 \%$ of the population comprising of slum dwellers,

Income:

All Bombay BPL (upto Rs: 1000 ) $74 \%$ of households

Slums

Rs.1000 p.m. (1976) $93 \%$ of households

Literacy:
All Bombay
Male
$77 \%$ literate
Female $\quad 65 \%$ literate

Major Air Pollution Sources:
Automobiles
Industries
Fùel burning
Solid waste dumps
Wind blown dust

$60 \%$ is due to vehicular exhaust. The gravity of auto exhaust is resulting from inadequate roads leading to reduced speed and large proportion of old vehicles, and increase in total numbers.

Permissible level of carbon monoxide is $3 \%$ for petrol engine and $4.5 \%$ for diesel engine. The tests show that $80 \%$ of the petrol vehicles exceeded the limits and reached $8 \%$ of carbon monoxide. The diesel vehicles showed similar results.

\section{Solid Waste Quantity:}

$\begin{array}{ll}\text { Domestic waste } & 400 \text { tonnes per day } \\ \text { Debris excavation construction } & 1500 \text { tonnes per day } \\ \text { Collection points } & 4000 \\ \text { Wastes transport } & 800 \text { vehicles per day }\end{array}$

The garbage contains, $10 \%$ paper, $2 \%$ plastic, $30 \%$ ash/earth, $35 \%$ compostible materials. $\mathrm{PH}$ value of 7.8 . Cost of solid waste 
management in Bombay is Rs.15 per capita. The local authority is responsible for collection, transport and disposal of garbages.

BMC Water Supply:
Domestic Demand
Domestic Supply
3360 mld.
Shortage
$2500 \mathrm{mld}$
$860 \mathrm{mld}$.

Drinking water is safe because of the distance of water sources from the main city. Most water pollution in old buildings and slums occurs because of the leakages in the pipe line due to rusted pipelines, or breakage.

Per Day Per Capita Availability of Water:

General population120 1trs.

Slums 50 itrs.

Toilets:

Approximately $50 \%$ of the slum population either have no toilets or have unusable toilets. The city needs more than 40,000 toilets to achieve the tolerable ratio with the population (1:25 families).

\section{Health Status:}

\begin{tabular}{lrr} 
& General Population & \multicolumn{1}{c}{ Slum } \\
\cline { 2 - 3 } & & 12.3 \\
DIARRHOEA & 10.1 & 9.7 \\
BREATHLESSNESS & 6 & 21.8 \\
COUGH & 20.7 & 38.1 \\
COMMON COLDS & 23.8 & 3.4 \\
BLOOD PRESSURE & 8.8 &
\end{tabular}

Noise Pollution:

During day minimum noise level $55 \mathrm{~dB}$.

During night minimum noise level $45 \mathrm{~dB}$.

Residential area along roads during the day $65-95 \mathrm{~dB}$.

Average noise level $80 \mathrm{~dB}$. 


\section{A Brief Note on Public Distribution System}

The Public Distribution System is a Central Government Scheme initiated in the country during the period of shortages in the IInd World War. The scheme continues till today, as it is part of the pledge of the Government to the poor. Through this scheme subsidised foodgrains (wheat, rice), sugar and kerosene, the major fuel, are supplied to the consumers at predetermined prices and fixed quotas per unit. Occasionally other items such as cooking oil, cereals, etc. are also made available.

As in October 1994, there are 3,022 Fair Price Shops in Thane and Bombay, catering to $3,471,288$ families in the region. It is claimed that around 25,000 new cards are being issued every month. The whole system operates through an elaborate network with Government food stock godowns, ration-shops and the bureaucracy as major actors. At the community level, officers are responsible for issuing new cards, addition of new names, increasing existing units and cancellation and transfer of cards.

The recent efforts of collaboration have helped to enable NGOs and CBOs to interact with relevant officers, who play a crucial role in communities. Joint consultations, monitoring of shops, setting up of vigilance cells and broad based community education with active collaboration of the Department have been the major outcomes of this effort. 



\title{
PRINCIPLE CENTRED DEVELOPMENT: A NEW PARADIGM HABITAT
}

\author{
SHEBA HUSSAIN
}

The year 2000 will see half the people of the world living in cities. Urbanisation of society is part of the development process with cities generating 60\% of the GNP. However, government support in services to growing cities has not increased proportionately. Urban habitats are therefore beginning to face a serious global environmental and development crisis.

The major cause of the continued deterioration of the world's environment is the unsuitable pattern of consumption and production, particularly in industrialised countries. Excessive demands together with the insensitive, wasteful life styles of the richer segments of humanity place an immense strain on the environment, the resources being exploited and degraded with impunity. On the other end, poor societies are unable to meet health care, shelter and educational needs - thus aggravating poverty and often perpetrating it.

While sustainable consumption patterns are needed, galloping population together with unsustainable consumption habits are putting an enormous pressure of stress on the earth's resources. Population growth must necessarily be dealt with by development strategies which safeguard the health of the system by promoting environment friendly technologies and access to resources on an equitable basis. The primary goals of sustainable development must include appropriate poverty alleviation measures which provide opportunities for obtaining secure livelihoods, sound health and a quality of life which meets basic needs. Further these measures have to focus more on women who are comparatively more vulnerable and exploited.

Urbanisation is as old as civilisation. The form and structure of urban habitats reveal the manner in which the challenges posed by the growth demands of society were responded to by the city at each stage. These changes while serving the political and economic interests of the privileged elite and politically powerful groups, paid scant attention to the needs of the poor and marginalised segments of the population.

The experience of development planning has clearly indicated that, to achieve growth with equity, key prerequisites require to be established first: planning demands a positive attitude which reflects the human face of development; regulatory process should be such as to provide alternatives for growth, rather than to prevent or skew growth directions and benefits.

Most people derive their security from their membership in a group - a family, a community, an organisation, a racial or ethnic group - that can provide a cultural identity and a reassuring set of values through homogenous and mutually reinforcing interaction. There is a need to shift policies which value the security of technologies to those which value the security of human society with an inherent concern for living life with dignity and peace. This concept which 
appears simplistic is indeed difficult to actualise - its implementation through political will backed by operational support would revolutionise life in the 21 th century. Principle leadership recognises that the wellbeing and peace of the global community per se would be more easily achieved through timely and proactive containment rather than late and reactive interventions.

While poor people have less health security, the situation of women is particularly difficult - the most serious hazards being childbirth, access to safe and affordable family planning and basic support at home during pregnancy and delivery. A miracle of life often turns into a nightmare of death just because a society cannot spare the loose change to provide a birth attendant at the time of greatest vulnerability and anxiety in a woman's life.

Personal insecurity shadows women from conception to the grave - in the household they are the last to eat, at school the last to be educated, at work the last to be hired and first to be fired and from childhood to adulthood the butt of exploitation just because they are women.

\section{FAMILY AS AN ENVIRONMENT}

The family can be seen as an environment made up of people and their immediate physical surroundings. Individuals who are members of a family interact on a daily basis. A family is a structure that is a set of relationships and a space that creates that environment within which the social, psychological, physical and economic needs of individuals are constantly interacting. The totality of solutions to everyday life problems determine the quality of life.

The family is an environment situated within the larger sphere of society and the two environments interact constantly affecting one another. There is a need to be aware of, and sensitive to, and to be in touch with the realities of social functioning around the world as they do, in no small measure, impact on the life styles of people seemingly distant and diverse from them.

\section{FAMILY AS PART OF THE ECOSYSTEM}

Individuals who make up the environment must constantly adapt and change. By seeing themselves as a part of the global ecosystem, families can learn to be responsible to their environment, understanding that a reverence for life is dependent upon a concomitant reverence for its condition.

An ecological approach that places the emphasis on reviewing the family and the environment holistically allows one to note the interdependence between people, families to one another and to other social systems, especially the interdependence of families to the realities of the natural environment.

\section{DEPENDENCE, INTERDEPENDENCE}

In the context of development, and the passage of time, the global family can transit from dependent components to independent entities and family to a interdependent, synergetic whole. The global synergy is the harmony between the clock and the compass - the clock 
representing the management and utilisation of time and the compass representing the direction or how we lead our lives. This development paradigm which celebrates human effectiveness is governed by principles or natural laws in the human dimension. Principles are guidelines for human conduct that are proven to have enduring, permanent value.

Absolute poverty, rapid population growth and environmental degradation are wreaking havoc throughout much of the developing world today. All these problems have been addressed separately when they have been addressed at all - with limited resources, and not surprisingly, limited impact. In fact, they have become so inter related, so mutually reinforcing that approaching these problems separately risks underestimating the severity of the crisis they represent taken together.

\section{THE SOUTH AND ITS TASKS}

In this last decade of the century the world is in a process of rapid transition. Political alignments, economic systems, and social values are being transformed. Conventional notions are less and less applicable to the changing international environment. These momentous changes present opportunities from which the South should seek to benefit. Acceleration of science and technological advance are at the base of these changes. These affects societies, economies and international relations at a fundamental level. Technology advancement at an unprecedented rate towards the globalisation of economies has given a strong impetus to trends being induced by such institutional changes as deregulation and privatisation undertaken by the leading countries of the North.

The South covers the larger part of the earth's surface. Its people are the vast majority of the world's inhabitants. But they have a very much smaller proportion of the world income. The challenge to the South is to reaffirm in words and in action, that the purpose of development is the promotion of the wellbeing of its people, with economic growth directed at their needs and fulfilling their aspirations.

Most developing countries will need to expand their economies at a fairly rapid rate to satisfy the legitimate requirements and desires of its people. The process of growth will entail a significant increase in the use of natural resources to which the international community will have to adjust if the South is to attain its development goals without harming global ecological stability.

A critical factor is how the South itself confronts the challenge in its choice of development patterns and life styles. Neither the demand side nor the supply side of these patterns can simply be a replica of the past or a blind copy of existing models of advanced industrial societies.

The challenge of removing extreme poverty can become a reality only if a commitment is made to meet basic human needs through people centred strategies for development. Economic growth and the meeting of basic needs have to go together.

The South's development strategy must aim at satisfying basic human needs and at closing the distance between the North and the South in food, education, health, safe drinking water and a healthy and secure environment. Science and technology can be powerful instruments for regenerating economies. In more populous countries, dispersed, broad based and regionally balanced 
development is particularly important to avoid the unplanned and unregulated growth of massive urban agglomerations which become increasingly chaotic and ung overnable.

\section{A HUMAN CENTRED STRATEGY FOR ENVIRONMENTAL IMPROVEMENT}

A human centred strategy or primary environmental care is an integrated community based approach aimed at meeting basic needs through the empowerment of people and protection and optimal use of the national resources on which people's livelihood depends. Priority should be accorded to meeting basic human needs in an environmentally sustainable manner and to the empowerment of communities by sensitising the targeted communities to environmental issues in the process.

Strategies and actions must be critically judged by the impact they have on people at the community and the household levels. This would be for example, the impact on sectors such as child health and development, water and sanitation, food security, education, employment, household energies and income generation. These sectors form synergistic parts of an integrated whole, the whole in this case being the wellbeing of the community.

Community participation will be vital to ensure that the assessment of problems, formulation of strategies, implementation, monitoring and evaluation all take into account the environment at the local level. Cost of effective social mobilisation, and adaptation to local cultures and priorities are also essential. Efforts are only sustainable when the benefits are tangible at the community level. In order to sustain community level change, however, national level policies must form a favourable network.

The kind of local level human-focused environmental strategy outlined here should take place simultaneously with the actions on the broader issues such as debt, structural adjustment, and environmental change. Only then can we hope to make an impact on poverty and environmental degradation.

\section{WOMEN IN SUSTAINABLE DEVELOPMENT}

A new development paradigm in the current context of the environmental crisis and the blatant failure of the old development paradigm to meet the needs of women and the poor, is required. Far from eradicating poverty, the growth model of development has impoverished many women and local communities in both rural and urban sectors.

Sustainable development is sustainability for whom? Sustainability for what? Sustainable development should not be sustained profits for private and public sector interests at the expense of women, the poor and indigenous communities. Sustainable development is the sustained livelihood of people embedded in an environment able to renew itself.

A new development paradigm must be for mulated in recognition that sustainable modes of living in a self renewing environment, proven viable, are already being practised by communities in rural and urban settlements and that women's indigenous knowledge from these communities is incorporated in decision making and development planning. 
Women have considerable knowledge and experience in managing and conserving natural resources. However, the role of women in achieving sustainable development has been limited by barriers such as discrimination, lack of access to education, land and equal employment.

\section{Human DeVelopment Index (HDI)}

A Human Development Index is needed because national progress tends to be measured by the GNP alone. This more comprehensive socio-economic measure has three basic components of longevity, knowledge and standard of living. One of the most important difference within the overall HDI score for any country is between men and women. Men generally fare better than women on almost every socio-economic indicator. For example, the average sex ratio in India is 927 per 1000 men, but within developing countries, several states e.g., the most populous state U.P. has only 882 women per 1000 men. The discrimination is board-based, it occurs not only in employment, but also in education, nutritional support and health care. Illiteracy is always higher for women. Neglect of women's health and nutrition is so serious in some countries that it even alters women's natural biological tendency to live longer than men. Considering their early deaths as well as those from infanticide, some studies estimate that up to 100 million women are "missing". The struggle for women's development has to be conducted by women not in opposition to men but as part of the social development of the entire population.

Gender inequality is both a development problem and an obstacle to progress in all developmental programmes. The most basic concept of development is that of overcoming poverty. Women are a majority of the poor. A woman faces pervasive gender discrimination in access to productive resources, making her self reliance difficult. If equality between man and woman's development is intrinsic to the definition of women development, the necessary corollary to it is women's empowerment, as a means to overcome the obstacles to women's equality in patriarchal societies.

In the analysis of the socio-economic situation of women relative to men, issues of women development assume significance when there are gender gaps at each of the interrelated levels of equality. These levels of equality are:

\section{WOMEN'S EMPOWERMENT FRAMEWORK}

The gender gap in the level of equality, relating to welfare happens when women are seen as passive recipients of welfare benefits, not as individuals capable of changing their lives. Women improve-ment cannot take place only at the welfare level.

Women's lower levels of productivity arise from their restricted access to the resources for development and production availability. The gender gap here refers to lower utilisation of opportunities and resources. Overcoming gender gaps will mean that women have equality of access and empowerment means that women are made aware of the differential situation and assisted to take action for gaining access to means and opportunities.

Often the lack of access to resource is a result of systems of gender discrimination when obstacles to access are a result of systemic discrimination, which can be addressed only through the empowerment process of conscientisation. 
The gender gap in women's participation is the most visible and obvious phenomenon.

Participation in a de velopment process would mean women being represented in the process of need assessment, problem identification, project planning, management, implementation, and evaluation. Equality in participation at a community level means involving women in the same proportion of decision making as their proportion in the community at large. This is not easily possible in a patriarchal society. Increased mobilisation of women will be needed to push for increased representation.

At the level of control the gender gap is manifested as the unequal power relations between women and men. Equality of control means a balance of power between women and men so that neither is in a position of dominance. The process of empowerment is self propelling and self reinforcing. Success at one level provides a better basis for success at other levels.

\section{THE INFORMATION AND COMMUNICATION REVOLUTION - ITS IMPACT ON THE EMPOWERMENT FRAMEWORK}

The world - especially the Asian region - is experiencing a veritable revolution in the field of information and data dissemination through the exploitation of modern technology. The combination of media with high state of art technology has put in place unprecedented and powerful means of spreading the widest possible range of information to the widest possible mass of people transcending linguistic, cultural and national borders. Clearly there is an urgency to take heed of the unlimited possibilities of fered by the current electronics communication technology for all those with a serious agenda in information dissemination and development.

Information is now a crucial determinant of the pace of social and economic change. Very often, the manner in which data are collected, classified and organised has far reaching implications for the use that is made of these data.

The growing consumerist movement has been fed and promoted through the same revolution of information and communication. With short sighted visions and privatised goals, unchecked communication methods and modes have contributed greatly to the weakening of the social fabric. It has generated conflicts in human security by attempting to interpret traditional value systems and often reinforcing harmful attitudes and practices.

Many people do not understand the close ties between human activities and the environment due to inaccurate or insufficient information. There is a need to increase people's sensitivity to an involvement in finding solutions for environment and development problems. Education can give people the environmental and ethical awareness, values and attitudes, skill and behaviour needed for sustainable development.

There is already a wealth of information that could be used for the management of sustainable development but many people have trouble in finding the information they need, when they need it. In the developing world, the gap in the availability, quality and accessibility of data has been increasing. We need to use environmental, demographic, social and developmental information to identify indicators that show us if we are moving towards the creation of a more sustainable world. 
The existing co-operation among the media of developing countries should be greatly intensified and diversified and the necessary infrastructural links improved. Action on these lines would greatly contribute to the forging of horizontal links with the South for reducing dependence on the North based global information services and to diversify avenues of information in developing countries.

What is assumed in this paper could not be more sincerely articulated than in the seven major constraints articulated by the former Executive Director of UNICEF, the late Mr. James Grant who provocatively called these "The Seven Sins of unplanned Development":

1. Development without infrastructure,

2. Development without participation,

3. Development without women,

4. Development without environment,

5. Development without the poor,

6. Development without the do-able,

7. Development without mobilisation.

In the words of Elizabeth Stanton: "The prolonged slavery of women is the darkest page in human history"; further the words of the great Indian poet philosopher, Rabindranath Tagore who, while speaking on freedom in a different context, articulated that which could well apply to the freeing of women from stifling gender dominance. The Master gave a challenging call by his words "Arise Awake and stop not till the Goal is achieved", So be it.

\section{REFERENCES:}

DE SOUZA Alfred (ed.), Urban growth and urban planning, New Delhi, Indian Social Institute, 1983.

GRANT James P., "Address in UNICEF Lucknow", mimeo, 3 February 1995.

KEATING Michael, Earth Summit - Agenda for Change, Geneva, Center for our Common Future, 1993.

UNDP, Human Development Report, New York, 1994.

UNICEF, Gender training module, gender responsive programme implementation, women's empowerment frame work, New York, UNICEF, undated.

UNICEF, note C.F/EXD-IC/1991-026, September 1991.

United Nations, Family: Forms and functions, New York, Occasional papers, Series no. 2, 1992.

United Nations, Family as an environment: An ecosystem perspective on family life, New York, Occasional papers, Series no. 5, 1993.

United Nations, Proceedings of the Asian and Pacific Symposium of NGOs on Women in Development, Manila, 1993. 


\title{
WOMEN ENTREPRENEURSHIP IMPROVING THE QUALITY OF LIFE
}

\author{
RUNA BANERJEE
}

\section{THE CONTEXT}

Women of the developing world question the pursuit of economic growth as the model of development which has resulted in environ-mental degradation, increasing poverty and gender inequality. Faced with these crises, they need to build a new, environmentally sustainable, vision of development centred on the rights and needs of people. Here we will focus mainly on women strategies for the development of almost half the human population, i.e. women's call for new ethics, accountability, processes and mechanisms.

\section{FORWARD LOOKING STRATEGIES FOR WOMEN'S DEVELOPMENT IN THE FOURTH DEVELOPMENT DECADE 1990-2000}

The three objectives of this decade are equality, development and peace. These are broadly interrelated and mutually reinforcing so that the achievements of one contributes to the achievements of the other.

These goals are inextricably linked to the three subtopics of employment, health and education. They constitute the concrete basis on which equality, development and peace rest. The attainment of these goals is the concern of men and women, and society as a whole. Women must necessarily play a central role as intellectuals, policy makers, decision makers, planners and contributors, but also be the beneficiaries of development. Despite the considerable progress achieved and the increasing participators of women in society, this decade has only partially attained its goals and objectives. The overwhelming obstacles to the advancement of women are caused by varying combination of political and economic as well as social and cultural factors.

Within the context detailed above, efforts to promote the economic and social status of women should rely on the International Development Strategies and the principles of the New International Economic Order. These principles include self-reliance and activations of indigenous human and material resources. The structuring of the world reviewed on a long term basis is to the benefit of all people.

According to ILO estimates and projection, women constitute $35 \%$ of the world's labour force and this figure is likely to increase steadily by the year 2000 . Women have the sole responsibility for the economic support of a large number of the world's children - approximately one third and higher in some countries. While women's total input of labour in the formal and informal 
sector will surface that of men by the year 2000, they will receive an unequal share of the world's assets and income.

\section{THE INDIAN SCENARIO: RURAL-URBAN MIGRATION OF WOMEN}

Patterns of female rural-urban migration in India have received little attention by either researchers or planners. While economic factors are obviously basic to migration decisions, social cultural factors give migration streams particular shapes in the Indian context. There appears to be significant differences in sex-ratios among migration patterns originating from different parts of the country, and even between different castes within a particular region.

An analysis of data at the national level reveals that there are differences in reasons between men and women for migrating to urban areas. According to several reports $46 \%$ of women migrated after marriage, compared to $0.6 \%$ of men, while $40 \%$ of men migrated for employment compared to only $2.7 \%$ of women. In addition $28.4 \%$ of the women migrated with an earning member or as a dependent of the household compared to $18.5 \%$ of men. The percentage of men migrating for studies, under transfer on service or business contract and for political reasons, was far higher than that for women. In terms of the size of the city, the number of those migrating for employment and for political reasons was greater for the metropolitan cities than the medium and smaller areas.

\section{URBAN POVERTY, WOMEN AND EMPLOYMENT}

A study by Andrea Menefee Singh reveals that it is mostly pure economic necessity which brings poor urban women into the worked force. As a woman approaches middle age and her children grow and become more expensive to support, it is increasingly difficult to meet the minimum needs of the family on just one person's income; therefore income supplementation becomes imperative. Moreover, women who are widowed, deserted or otherwise forced to be the sole or primary providers of income for themselves or their families are increasingly joining the work force, largely in the informal or unorganised sector.

While the decision to enter the work force is almost always economically motivated, both caste and regional factors exercise an important influence. There are at least four main reasons which influence the types of jobs which women can undertake:

- Recruitment to jobs in the unorganised sector is almost always done through informal social networks.

- Women's traditional work in the village prepares them with skills which are adaptable to the urban employment market.

- Women whose traditional caste occupations are considered polluting may find it difficult to change to another job's type.

- Caste and regional values tend to dictate the kinds of work that a woman should or could do. 


\section{AgENDA FOR CHANGE}

Policies programs and projects aimed at or incorporating especially vulnerable and marginalised women in the urban context will have to recognise the difficulties of removing the multiple obstacles facing such groups and must place equal emphasis on addressing the social, economic and human dimensions of their vulnerability and their underprivileged positions.

While governments have the obligation to organise multisectorial programmes with an emphasis on economic activities, elimination of gender discrimination, the provision of supportive services, NGOs other networks and most importantly women themselves need to be empowered to participate equitably in decision making which is imperative for increasing their self-reliance and holistic development.

\section{PARTNERSHIPS}

A. Non-governmental organisations play a vital role in shaping an implementation of participatory democracy. Governments are increasingly involving NGOs in sustainable development plans, making the best use of their abilities in such areas as education, poverty alleviation, environmental protection and rehabilitation. The findings of NGOs are being used, but must be increasingly tapped for shaping policies on sustainability.

B. Local authorities such as municipal governments build and maintain structures such as drinking water systems, roads, sewers, health care facilities, housing and industries. While doing so they set local environmental policies which reflect global and national priorities. All these however need to be much more gender sensitive in their approach.

C. Workers Trade Unions - Unions and employees should in consultation with women design joint environmental policies, and set priorities to inquire the working environment and the overall performance of business and industry. Women through employment should form trade unions which would contribute to the formulation of sustainable development policies and collective agreements aimed at improving the overall quality of life.

D. Business and Industry - Responsible entrepreneurship can play a major role in improving the efficiency of resource usage, minimising waste and protecting human health and environmental quality. The evolution of human settlements which respond to the needs of women must be given the priority they demand. To ensure this, business and industry need to develop techniques and technologies that not only reduce harmful environmental impact but are appropriate for women and free them from existing drudgery.

E. Women's growing advocacy and pro-active participation : the key milestone

- Article 2 of the Universal Declaration of Human Rights proclaims that everyone is entitled to all the rights and freedoms set forth in this declaration without distinction of any kind such as race, colour, sex (1948).

- International Women Year: the World Conference at Mexico gives the call for elimination of all forms of discrimination against women (1975). 
- Nairobi : Forward looking Strategies for the Advancement of Women (1985).

- Cairo (Population) and Copenhagen (Social Development) for achieving end decade goals while projecting perspectives for the coming millennium (1994).

- Beijing 1995: Fourth World Conference on Women and NGO Forum on Women building on Rio (Agenda - 21).

\section{SELF EMPLOYED WOMEN's AsSOCIATION (SEWA) LUCKNOW} (see annex)

\section{OBJECTIVES :}

- To ensure that chikan craftswomen get sufficient work at fair wages without exploitation by middlemen.

- To identify and de velop promising new markets for chikan products.

- To organise chikan workers by developing their confidence, leadership potential and sense of security.

- To train members in purchase, production, management, marketing and accounting.

- To upgrade the skills of artisans through training programmes which improve the quality and range of their work.

- To revive and revitalise the traditional craft and take it to its original level of refinement.

- To provide a strong platform from which artisans can bargain for higher wages from traders and middlemen.

- To ensure social benefits like educational and health facilities for members and their children.

\section{ACHIEVEMENTS:}

- The economic and social empowerment of its artisan members which have grown from the original 31 to the present 4000 .

- Elimination of middlemen/contractors as also release from "bondedness".

- Revival of the craft which has now showed an overall improvement in quality with a positive reciprocity in demand increase.

- Increasing access to credit for meeting consumption and produc-tion needs.

- Increasing outreach beyond and the core SEWA membership by the formation of 38 self-help groups (SHGs) having a membership of over 500.

- Environmental improvement through community managed water and sanitation interventions.

- Provision of basic education through adult literacy for women and non formal education with a focus on girls' education.

- Health care mainly through a referral system.

- Employment through information dissemination regarding legal and other rights.

- Establishing systems which strengthen individual and corporate growth through mutually reinforcing synergy.

- Increasing the bargaining power of artisans.

- Improving the status of women in families and communities with visible improvement in marketing. 


\section{Perspectives:}

- Providing a platform for articulating the need for recognising and meeting the felt needs of women workers of the unorganised sector.

- Working for the objective of women having an increasing control on their reproductive health by planning their families.

- Advocacy for providing women the necessary social security through insurance, pension and implementation of existing legislation protective of women's rights.

- Networking for getting political commitment which would protect urban habitats and ensure that the objective of sustainable human de velopment is achieved.

\section{REFERENCES :}

DE SOUZA Alfred (ed.), Urban Growth and urban planning, New Delhi, Indian Social Institute, 1983.

KEATING Michael, Earth Summit - Agenda for Change, Geneva, Center for our Common Future, 1993.

United Nations, Proceedings of the Asian and Pacific Symposium of NGOs on Women in Development, Manila, 1993.

\section{ANNEX \\ SEWA SELF EMPLOYED WOMEN's Association, LuCKNOW}

SEWA continuously strives to meet its objectives. The growth of the organisation in the last tyears has been tremendous as is shown below:

\begin{tabular}{lrrrr}
\hline Année & $\begin{array}{r}\text { Nombre } \\
\text { de membres }\end{array}$ & Ventes $(\$)$ & $\begin{array}{r}\text { Achat de } \\
\text { mat. prem. }(\$)\end{array}$ & Salaires $\$$ () \\
$1984-85$ & 163 & 3,532 & 4,898 & 2,538 \\
$1985-86$ & 170 & 5,581 & 2,914 & 3,404 \\
$1986-87$ & 200 & 7,579 & 6,572 & 4,260 \\
$1987-88$ & 250 & 12,442 & 9,166 & 9,937 \\
$1988-89$ & 375 & 23,527 & 16,630 & 14,249 \\
$1989-90$ & 450 & 72,665 & 49,383 & 30,712 \\
$1990-91$ & 800 & 269,403 & 91,107 & 104,293 \\
$1991-92$ & 1300 & 439,679 & 237,884 & 229,625 \\
$1992-93$ & 3000 & 375,616 & 306,583 & 346,681 \\
$1993-94$ & 3500 & 738,457 & 276,945 & 315,733 \\
$1994-95$ & 4000 & 607,032 & 119,829 & 284,666 \\
$(3$ trimestres) & & & & \\
\hline
\end{tabular}




\section{ECONOMIC ACTIVITIES}

SEWA's greatest strength lies in the trust and respect it has earned from the community and SEWA has done extremely well in improving the economic status of its members. Artisans are paid among the highest wages in the State and even the Government has responded by raising wages at the Government Production Centres as well. Recently, SEWA has diversified into other handicrafts like tie-and-dye, block printing, mirror work and appliques. SEWA hopes to bring new life to these traditional Indian handicrafts by combining them in new and exciting ways. With chikan as the leitmotif, popular interest and demand is certain to be revived in these other art forms. In an effort to expand SEWA's outreach, increase production and decentralise its activities, rural training-cum-production centres are being set up in four villages of Lucknow District to be started and operated entirely by SEWA members. Linkages have also been developed with the U.P. Government, with SEWA agreeing to absorb 370 TRYSEM (Training of Rural Youth for Self Employment) and DRDA (District Rural Development Agency) trained women as members. Marketing efforts have intensified with the number of exhibition-cum-sales increasing from two annually (in New Delhi and Bombay) to four (in Bombay, Chandigarh and twice in New Delhi) in 1991-92. In addition SEWA has just opened its exclusive retail shop in the prestigious Hazratganj shopping locality of Lucknow.

\section{TRAINING \& EDUCATION}

Training is an integral part of SEWA's activities and the organisation is constantly involved in teaching and improving the skills of its members in all aspects of the craft. SEWA has recently self-financed eight classes in chikan and zardozi embroidery block printing, tie-and-dye, mirrorwork.

Beginning June 1992, SEWA intensified its efforts to reach rural women. More than 1000 needy women belonging to the low-income strata of society have been trained in Chikankari in the districts of Lucknow, Unnao, Baranbanki, Sitapur and Bahraich. After completing their training, the women have become regular members of SEWA, with regular earnings. More than 450 women are being trained in the districts of Lucknow, Sitaprur and Kanpur. This training is being imparted with financial assistance from Government programmes (DRYSEM, DWACRA AND NABARD) and UNICEF.

A vocational training programme in 'Asri Zardozi' embroidery has been launched for older children in the SEWA school which now has an enrolment of over 650 students. The promise of SEWA membership for those who complete the one-year vocational training should motivate the children to continue with their studies until they complete at least seven to eight years of schooling. They are sponsored entirely by Bharat Petroleum Corporation Limited.

SEWA has also assumed a prominent role in non-formal education in Lucknow. All of SEWA's members are encouraged to learn to read and write, and to acquire a working knowledge of basic arithmetic. A glance at SEWA's wage payment registers where one can trace the growing functional literacy of its members by the near-total elimination angutha-chhap (thumbprint) signatures provides proof of SEWA's success in the field. SEWA has assumed a leading role in the community programme "Sunder Lucknowr Sakshar Lucknow" (Beautiful Lucknow literate Lucknow) which aims to beautify the capital and make it fully literate in one-year's time. SEWA teachers and staff members are required to spend time in extension activities promoting adult literacy. This further develops their leadership potential and reinforces their sense of commitment to community development. 


\section{WATER \& SANITATION}

SEWA has also undertaken a UNICEF aided 2-year project (to be completed in November 1994). Four blocks in Lucknow, Sakori Mal Gosaiganj and Chinhat -comprising 28 villages are covered under this project, involving the installation of INDIA MARK III pumps. Another aspect of this project is the formation of the PARYAVARAN SUDHAR SAMITIs

(Environment Upgradation Committees) to ensure sensible and equitable utilisation of water. These committees comprise 5 women and 2 men including the Pradhan (Headman) of the village. The villagers pay between Rs. 2/- and Rs. 5/- per family for each month and the accounts are maintained by these committees. Further, one caretaker is appointed for each of the pumps (usually a woman) including for non-SEWA pumps. The caretakers are trained in the care and use of the pumps by SEWA. It is envisaged that very soon, mechanical training for the maintainance of these pumps will be imparted and many villagers have sought to be trained. Also, training is given in the development and use of simple but effective sanitation techniques, both for respective homes and for the localities. All in all this project involves the participation of 250.000 people, many of them children.

\section{SAVING AND CREDIT PROGRAMMES}

The programme of savings is linked to the Revolving Credit Fund Programme for SEWA members. This programme which was launched recently makes small loans available to chikan artisans who wish to engage in supplementary/alternative income generating activities. In addition, loans are also provided for desperate and immediate needs like medical treatment, construction of house etc. Recognising the need to keep the programme running on a long term sustainable basis, SEWA is disbursing loans on interest so that the interest earned can be channelled back into the revolving fund and meeting the services costs incurred during the implementation of the credit programme.

\section{HEALTH SERViCES}

In the past SEWA has run several health camps for its members. With the help of a grant from the Canadian Embassy, SEWA has started a health clinic where members and their families receive continuous professional care and assistance. At present four specialist doctors, an ophthalmologist, a general physician, a gynaecologist and a paediatrician from King George Medical College Lucknow pay a visit to SEWA clinic once a week. In future SEWA plans to extend basic Primary Health Care and medical facilities in different villages where it has built contacts over the last ten years.

\section{STREET CHILDREN}

With increasing urbanisation of Lucknow City there has been a phenomenal rise in the number of street children below the age of 15. Most of these street children are involved in activities such as rag picking and begging. Recognising the fact that these children do not have access to formal education and are a source of cheap Child Labour due to poverty, SEWA has started a programme of informal education with them. Along with education SEWA provides vocational training to street children so that they can earn a regular income in the long run. As part of this effort, SEWA will make these children environmentally conscious and train them in recycling waste material for packaging etc., a lot of which SEWA uses. 


\section{WEAVING}

From December 1993, SEWA has started a project with the weavers in the village of Baragoan in the District of Barabanki. Though this project is recent 5 weavers are regularly producing handloom fabric on which SEWA women do chikan embroidery before it is sold in the market (with only an initial investment of Rs. 12.000.00). SEWA is already paying the weavers twice the wages compared to what they receive from private traders. This is also an effort to make a direct linkage between the producers of the handloom fabric and the chikan workers.

\section{EMPOWERMENT}

SEWA has encouraged and developed self-confidence and leadership among its members partly by providing them with a sense of security but also by promoting awareness about their strengths and rights. The daily prayer session at the SEWA Production Centre always ends with calls for freedom from repression whether economic, political or religious. The women sing about equality and respect. That this goes beyond the words and slogan shouting can be seen in the fact that the SEWA Governing Body is composed entirely of women, that nearly $60 \%$ of SEWA staff members are artisans who have arisen to positions of responsibility by virtue of hard work and dedication and in the pursuit of education by some members even up to the post-graduation level.

\section{The ETHEL GRAnt SEWA CENTRE}

Construction of the new building was completed early in 1992 and SEWA moved in immediately even while the finishing touches were still being applied. All of SEWA's staff members participated both in Hindu prayers and a reading of the Koran before the move blessing the Ethel Grant SEWA Centre two times over - such was the excitement of SEWA members finally having a place to call their own. The gleaming, elegant white building is a source of pride for the artisans, symbolising sisterhood and strength. It has brought nearly all of SEWA's scattered activities together under one roof at last. SEWA is grateful to the Ethel Grant Foundation, USA for making this dream come true.

\section{FutURe Plans}

Most important of all SEWA's future aspirations is the need to be fully self-reliant. To accomplish this objective, SEWA has entered the export field since there is a huge demand for hand embroidered products in the West. At a recently held export fair in Delhi SEWA got a very encouraging response from foreign buyers, many of whom placed their orders with SEWA.

Regular cash flow is necessary for any organisation to function on a financially viable basis. Recognising this, SEWA plans to buy retail outlets in Delhi and Bombay like the one it has in Lucknow. As and when our financial resources permit us to achieve this goal, we would have taken a major step towards making SEWA financially self-sufficient.

And last but not least, SEWA requires to build a corpus fund of RSV. 1 core to enable it to become completely viable and dependent on its own resources. 